Simultaneous Photonic and Phononic Band Gap, Defect States and Waveguides in Silicon-Polymer Composite

By

Mohammed Alzahrani , B.Eng

A Thesis

Submitted to the Faculty of Graduate Studies and Research in Partial Fulfillment of the Requirements for the Degree of

Master of Applied Science

In Electrical Engineering

Ottawa-Carleton Institute for Electrical Engineering

Department of Electronics

Carleton University

Ottawa,Ontario,Canada

Copyright $(\mathcal{C}$ July 2012 by Mohammed Alzahrani 
Library and Archives

Canada

Published Heritage

Branch

395 Wellington Street

Ottawa ON K1A ON4

Canada
Bibliothèque et

Archives Canada

Direction du

Patrimoine de l'édition

395 , rue Wellington

Ottawa ON K1A ON4

Canada
Your file Votre référence

ISBN: $978-0-494-93489-0$

Our file Notre référence

ISBN: 978-0-494-93489-0
NOTICE:

The author has granted a nonexclusive license allowing Library and Archives Canada to reproduce, publish, archive, preserve, conserve, communicate to the public by telecommunication or on the Internet, loan, distrbute and sell theses worldwide, for commercial or noncommercial purposes, in microform, paper, electronic and/or any other formats.

The author retains copyright ownership and moral rights in this thesis. Neither the thesis nor substantial extracts from it may be printed or otherwise reproduced without the author's permission.
AVIS:

L'auteur a accordé une licence non exclusive permettant à la Bibliothèque et Archives Canada de reproduire, publier, archiver, sauvegarder, conserver, transmettre au public par télécommunication ou par l'Internet, prêter, distribuer et vendre des thèses partout dans le monde, à des fins commerciales ou autres, sur support microforme, papier, électronique et/ou autres formats.

L'auteur conserve la propriété du droit d'auteur et des droits moraux qui protege cette thèse. $\mathrm{Ni}$ la thèse ni des extraits substantiels de celle-ci ne doivent être imprimés ou autrement reproduits sans son autorisation.
In compliance with the Canadian Privacy Act some supporting forms may have been removed from this thesis.

While these forms may be included in the document page count, their removal does not represent any loss of content from the thesis.
Conformément à la loi canadienne sur la protection de la vie privée, quelques formulaires secondaires ont été enlevés de cette thèse.

Bien que ces formulaires aient inclus dans la pagination, il n'y aura aucun contenu manquant. 


\title{
Abstract
}

\section{Simultaneous Photonic and Phononic Band Gap, Defect States and Waveguides in Silicon-polymer Composite}

\author{
Mohammed Alzahrani
}

\begin{abstract}
A Thesis
Submitted to the Faculty of Graduate Studies and Research in Partial Fulfillment of the Requirements for the Degree of Master of Applied Science Carleton University
\end{abstract} 2011

A silicon-polymer composite is presented which supports simultaneously a band gap for optical and acoustical waves. The reciprocal nature of the composite's unit cell (high and low optical and acoustic properties) enables a full band gap for both optical polarizations to exist in Phoxonic crystal and at the same time an acoustic band gap. Square lattice structure is examined and the result details are presented. The propagation properties of a phoxonic waveguide, introduced into the structure by dielectric (polymer or silicon) shifting a gap along a lattice direction, are examined. The theoretical investigation is performed using the plane wave expansion method in both the optical and acoustical regimes. 


\section{Acknowledgements}

This work would not have been successful without the guidance and the help of several individuals.

Frist and most important I would like to thank my wife, Eman, for her love, support and great patience at all times. Also, especial thank to my parents, brother and sister have given me their unequivocal support for which my mere expression of thanks likewise does not suffice.

I would like to thank my supervisor, Prof. Robert Gauthier who was abundantly helpful and offered invaluable assistance, support and guidance.

I would like to thank my colleagues and friends in the Lab for their kindness, friendship and support. 


\section{$\underline{\text { Table of Contents }}$}

$1.0 \quad$ Introduction............................................................. 15

1.1 Motivation...................................................... 17

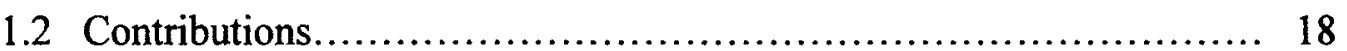

1.3 Applications..................................................... 18

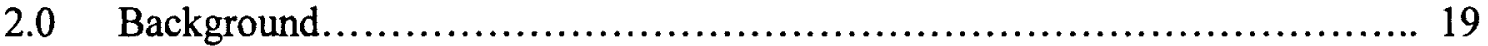

2.1 Introduction ..................................................... 19

2.2 Basic Wave Properties Governing Photonic and Phononic Crystals ..... 19

2.3 Phoxonic Structures.......................................... 26

2.4 Recent Developments in Phoxonics.................................. 28

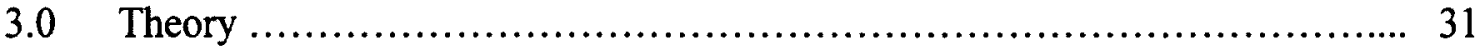

3.1 Introduction........................................................ 31

3.2 Maxwell's Equations....................................................... 31

3.3 TM and TE Wave Equations in the 2-D Photonic Crystal................. 33

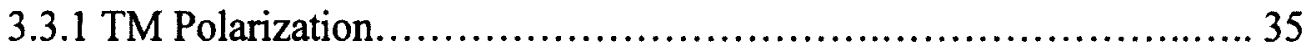

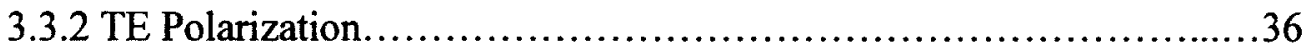


3.4 The Plane Wave Method...............................................38

3.4.1 One Dimension Photonic Crystal.....................................38

3.4.2 Two Dimensions Photonic Crystal..................................39

3.4.2.1 TM Application................................................. 42

3.4.2.2 TE Application..............................................43

3.4.3 MATLAB Programming of the Eigenvalue Equations...........................44

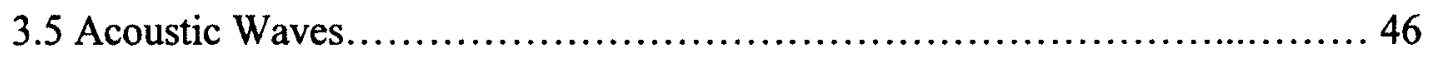

3.5.1 Plane Wave Method for the Acoustic Wave ...........................47

3.5.2 MATLAB Programming of Transverse Acoustical Equation ....................... 49

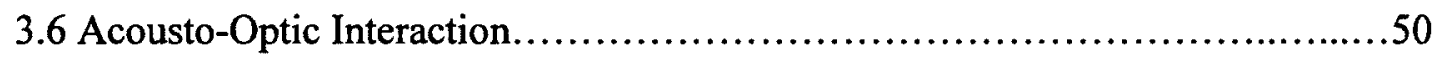

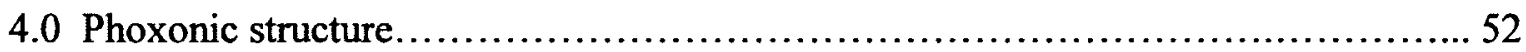

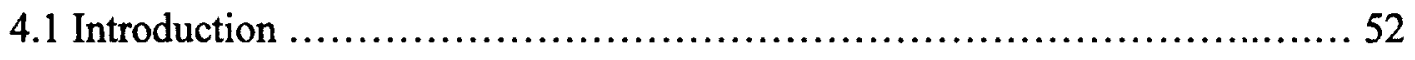

4.2 Different Shape of Inclusions............................................5

4.3 Solid-Solid Structure (Silicon and polymer) .................................55 
4.4 The Square Lattice Phoxonic Structure.

4.5 Bandgap Maps for Optical and Acoustical Domain. 65

5.0 Simultaneous Waveguide for Optical and Acoustic Waves. .73

5.1 Introduction .73

5.2 Supercell Method .73

5.3 Line Waveguide .78

5.4 Polymer as the Defect Material. .78

5.5 Silicon as the Line Waveguide. .86

6.0 Conclusion

7.0 Appendix

7.1 Appendix A : Simplifing and reduction of the summation terms of the Eigenvalue Equation

7.2 Appendix B: MATLAB Code for Opical calculation TE and TM. .94

7.3 Appendix C: MATLAB Code for Acoustic calculation shear mode 102

7.4 Refrences. 110 


\section{$\underline{\text { List of Figures }}$}

Figure 2.1: Constructive and destructive interferences in the forward and backward propagation directions, for one direction photonic crystals. $\varepsilon_{h}, \varepsilon_{l}$, are the high and low dielectric layers, respectively.

Figure 2.2: Yablonovite structure, reprinting with permission ${ }^{2}$; left: show how the Yablonovite structure is fabricated, right: Image Yablonovite structure using computer simulation.

Figure 2.3: Band gap in a peridoic material with high-dielectric-constant material $\left(\varepsilon_{h}=13\right)$ of width $0.2 \mathrm{a}$ and low-dielectric-constant material $\left(\varepsilon_{h}=1\right)$ of width $0.8 \mathrm{a}$. Reprinted with permission ${ }^{2}$.

Figure 2.4: A triangular lattice of air inclusions in a silicon matrix, which exhibits complete phoxonic band gaps when $r / a$ is around 0.45 . Reprinted with permission ${ }^{11}$.

Figure 3.1: Square (left) and triangle (right) lattice photonic crystals. Black and white represents different materials used in the crystals.

Figure 3.2: One dimension photonic structure. Black represent low dielectric and white represent high dielectric.

Figure 3.3: Bravais lattice for two dimensions square lattice photonic crystal. Black represent low dielectric and white represent high dielectric.

Figure 3.4: The symmetry points of the two dimensions square lattice ${ }^{2}$.

Figure 3.5: The band structure reproduce using my MATLAB code in Appendix B, for the same parameters as in (ref.2). (Top) :TM, (Bottom): TE

Figure 3.6: The band structure obtained using the MATLAB program of Appendix $C$ and the results published in Ref.40 .

Figure 4.1: Left: square and circle cross-section inclusions for one unit cell. Right: $3 \times 3$ array; 
white for high dielectric silicon $\left(\varepsilon_{h}=12.11504\right)$ and black for low dielectric air $\left(\varepsilon_{l}=1\right)$. The radius of the circle cross-section $r_{h}=0.2 \mathrm{a}$, and the half length of the square side is $L=0.3 \mathrm{a}$.

Figure 4.2: Complete optical band gap of the photonic crystal structure of figure 4.1. The radius of the circle cross-section $r_{h}=0.2 \mathrm{a}$, and the half length of the square side is $L=0.3 \mathrm{a}$. For high dielectric silicon $\left(\varepsilon_{h}=12.11504\right)$ and black for low dielectric air $\left(\varepsilon_{l}=1\right)$. Top: TE. Bottom: TM.

Figure 4.3: Optical band diagrams for the structure of figure 4.1. The radius of the circle crosssection $r_{h}=0.2 \mathrm{a}$, and the half length of the square side is $L=0.3 \mathrm{a}$. For high dielectric silicon $\left(\varepsilon_{h}=12.11504\right)$ and black for low dielectric air $\left(\varepsilon_{l}=2.4\right)$. Top: TE. Bottom: TM. Grey: band gap regions. No complete optical band gap.

Figure 4.4: Left: rotated square and circle cross-section inclusions for one unit cell. Right: $3 \times 3$ array; white for high dielectric (silicon $\varepsilon_{h}=12.11504$ ), and black for low dielectric (polymer $\varepsilon_{l}=2.4$ ). Radius of the centre circle is $r_{h}=0.25 \mathrm{a}$, and the half length of square diagonal is $L=0.37 \mathrm{a}$.

Figure 4.5: Optical band diagrams for the structure of figure 4.4. Radius of the centre circle is $r_{h}=0.25 \mathrm{a}$, and the half-length of square diagonal is $L=0.37 \mathrm{a}$. For high dielectric silicon $\left(\varepsilon_{h}=12.11504\right)$ and black for low dielectric air $\left(\varepsilon_{l}=2.4\right)$. Top: TE. Bottom: TM. Grey: band gap regions. Black: overlap band gap regions.

Figure 4.6: Modified hybrid crystal structure. The white regions are high-dielectric silicon $\left(\varepsilon_{h}=12.11504\right.$, mass density $2.33 \mathrm{~g} / \mathrm{cm}^{3}$, speed of sound $\left.5360 \mathrm{~m} / \mathrm{s}\right)$, the black 
regions are low-dielectric epoxy of polymer $\left(\varepsilon_{l}=2.4\right.$, mass density $1.1 \mathrm{~g} / \mathrm{cm}^{3}$, speed of sound $2650 \mathrm{~m} / \mathrm{s}$ ). Left: one unit cell. Right: $3 \times 3$ arrays. Radius of the centre circle is $r_{h}=0.26 \mathrm{a}$, radius of the corner circle $r_{1}=0.2$, and the half-length of square diagonal is $L=0.32$ a.

Figure 4.7: Band gap for the structure shown in figure 4.6. Radius of the centre circle is $r_{h}=0.26 \mathrm{a}$, radius of the corner circle $r_{l}=0.2$, and the half-length of square diagonal is $L=0.32 \mathrm{a}$. Top: TE. Bottom: TM. Grey: band gap regions. Black: overlap band gap regions.

Figure 4.8: Transverse acoustic band gap for the structure shown in figure 4.6. Radius of the centre circle is $r_{h}=0.26 \mathrm{a}$, radius of the corner circle $r_{l}=0.2 \mathrm{a}$, and the half-length of square diagonal is $L=0.32 \mathrm{a}$.

Figure 4.9: The lowest speration in the final unit cell. For nano scale fabrication this minimum is large enougth.

Figure 4.10: Optical band gap map for the structure shown in Figure 4.6, plotted as a function of the centre circle radius and the lattice constant. The half length of square diagonal is fixed at $L=0.32 \mathrm{a}$, and the corner circle is fixed at $r_{l}=0.2$. The vertical line is the design value for $r_{h} / a$.

Figure 4.11: Optical band gap map for the structure shown in Figure 4.5, plotted as a function of the corner circle radius and the lattice constant. The half length of square diagonal is fixed at $L=0.32 \mathrm{a}$, and the centre circle is fixed at $r_{h}=0.26$. The vertical line is the design value for $r_{l} / a$.

Figure 4.12: Optical band gap map for the structure shown in Figure 4.5, plotted as a function of 
the diagonal square size half length and the lattice constant $L / a$. The centre circle is fixed at $r_{h}=0.26 \mathrm{a}$ and the corner circle is fixed at $r_{l}=0.2 \mathrm{a}$. The vertical line is the design value for L / a. Figure 4.13: acoustics band gap map for the structure shown in Figure 4.6: Plotted as a function of the centre circle radius and the lattice constant. The half length of square diagonal is fixed at $L=0.32 \mathrm{a}$, and the corner circle is fixed at $r_{l}=0.2$. The vertical line is the design value for $r_{h} / a$.

Figure 4.14: Acoustic band gap map for the structure shown in Figure 4.6, plotted as a function of the corner circle radius and the lattice constant. The half length of square diagonal is fixed at $L=0.32 \mathrm{a}$, and the centre circle is fixed at $r_{h}=0.26$. The vertical line is the design value for $r_{l} / a$.

Figure 4.15: Acoustic band gap map for the structure shown in Figure 4.6, plotted as a function of the diagonal square size half length and the lattice constant L/a. The centre circle is fixed at $r_{h}=0.26 \mathrm{a}$ and the corner circle is fixed at $r_{l}=0.2 \mathrm{a}$. The vertical line is the design value for $\mathrm{L} / \mathrm{a}$.

Figure 5.1: (left) $1 \times 4$ unit supercell, (middle) $1 \times 4$ supercell with a defect, and (right) the $4 \times 4$ array with the defect. Black represents the lower dielectric polymer, while white represents the higher dielectric material (silicon), and grey represents the waveguide material.

Figure 5.2: Optical TE band structure for a supercell (figure 5.1 left). The band gap is in the same location and of the same width as for the single unit cell (see figure 4.6 Top).

Figure 5.3: Optical TM band structure for a supercell (figure 5.1 left). The band gap is in the same location and of the same width as for the single unit cell (see figure 4.6 Bottom).

Figure 5.4: Acoustic band structure for a supercell without a defect state (figure 5.1 left). The 
band gap is in the same location and of the same width as for the single unit cell (see figure 4.7).

Figure 5.5: (Top) the number of modes inside the band gap as the polymer defect width increase. (Bottom) the number of modes intersected with the BG center as the polymer defect width increase.

Figure 5.6: TE mode, $\Gamma$ to $\mathrm{X}$ band structure for the structure that has polymer in the defect region, the width of the defect is $\mathrm{W}=0.5 \mathrm{a} .3721$ plane waves were used for this calculation.

Figure 5.7: TM mode, $\Gamma$ to $\mathrm{X}$ band structure for the structure that has polymer in the defect region, the width of the defect is $\mathrm{W}=0.5 \mathrm{a} .3721$ plane waves were used for this calculation.

Figure 5.8: $\mathrm{TM}$ mode profile at $\mathrm{NOBG}=0.78$ for the structure that has polymer in the defect region, the width of the defect is $\mathrm{W}=0.5 \mathrm{a} .3721$ plane waves were used.

Figure 5.9: $\mathrm{TE}$ mode profile at $\mathrm{NOBG}=0.78$ for the structure that has polymer in the defect region, the width of the defect is $\mathrm{W}=0.5 \mathrm{a} .3721$ plane waves were used.

Figure 5.10: Acoustic transvers mode band structure for the structure that has polymer in the defect region, the width of the defect is $w=0.5 a .5041$ plane waves were used.

Figure 5.11: Acoustic transfer mode profile at $\mathrm{NABG}=2680$ for the structure that has epoxy in the defect region, the width of the defect is $w=0.5 \mathrm{a} .5041$ plane waves were used.

Figure 5.12: (Top) the number of modes inside the band gap as the silicon defect width increase. (Bottom) the number of modes intersected with the BG center as the silicon defect width increase. 


\section{List of Symbols}

$\lambda$

f

$\vec{k}$

$\mu$

$\varepsilon$

$\mathrm{E}, \mathrm{H}$

$\mathrm{PtC} / \mathrm{PnC}$

PxCs

TE / TM

$\Gamma$

X
Wavelength

Frequency

The wave vector

The permeability of the material with subscripts further specifying its nature: $\mu \mathrm{o}-$ freespace

The permittivity of the material with subscripts further specifying its nature: $\varepsilon 0$ - freespace

Elecrtric field vector and Magnetic field vector, respectively

Photonic crystal and Phononic crystal respectively

Phoxonic crystal, photonic and phononic crystal simultaneously

Transverse electric and Transverse magnetic respectively

When labeled on the band diagrams this is a point of symmetry in the reciprocal lattice.

When labeled on the band diagrams this is a point of symmetry in the reciprocal lattice. 
When labeled on the band diagrams this is a point of symmetry in the reciprocal lattice.

PnBG

Phononic band gap

PtBG

Photonic band gap

(NOBG)

Normalized Optics Band Gap

(NABG)

Normalized Acoustic band gap

$a_{1}, a_{2}$

Lattice vectors

$l_{1}, l_{2}$

Integer numbers

$b_{1}, b_{2}$

Reciprocal lattice vectors

$\bar{X} \bar{Y}$

Periodicity plane

$\vec{u}$

Acoustic filed displacement

$\mathrm{Y}$

Young's modulus

$\rho$

Mass density 

respectively.

Force per unit area $\mathrm{A}$.

$r_{h}$

Radius of the centre circle

$r_{l}$

Radius of the corner circle

L

Half-length of square diagonal

W

The line defect of width 


\subsection{Introduction}

Periodic structures can occur naturally in nature in the form of crystals in which a basic building block is repeated over a lattice array ${ }^{1,2}$. When a wave propagates within the crystal a number of interesting effects can be observed with the most striking taking place when the wavelength is comparable to the lattice constant. In such cases, destructive interference of refracted and reflected waves can allow the exclusion of desired wavelengths. In solid-state electronics, the crystalline nature of silicon and the ability to dope the material with trace levels of impurities leads to a fine control over the energy levels of the electrons propagating in the material. The electron properties are typically displayed in what is known as a band diagram which illustrates the relationship that exists between the electrons frequency (energy E), propagation vector $(\vec{k})$ and type of crystal fabric (Brillouin zone) $)^{2,3}$. One of the most striking features displayed in the band diagram is the presence of a band gap region, a range of frequencies for which there is no corresponding wave vector and thus the energy levels within the band gap are not available. The presence of defects in the crystal and intentional doping result in a modification of the band structure and under the right conditions can introduce states within the original band gap. These states modify the material electrical properties and permit specialized device configurations to be possible 2,3

Artificial or manmade periodic structures have gained considerable interest over the last few decades. In the late 1980s the photonic crystal (PtC) was proposed as a medium that could influence light in a manner similar to that of the electrons in crystalline silicon ${ }^{1}$. At about the same time, the phononic crystal $(\mathrm{PnC})$ was proposed as a periodic medium that could influence the flow of phonons (sound waves of interest in this thesis) ${ }^{4}$. The theoretical tools used in the study of the wave nature of electrons in solid-state electronic have been carried over to the study 
of the wave nature in photonic and phononic crystals. The band diagrams for optical and acoustical waves are the most sought after design tool and correspond to displaying the optical (acoustical) frequency versus wave vector and crystal fabric ${ }^{2,4}$. The presences of defects in the artificial material can lead to states showing up in the band gap region ${ }^{2}$. When the defect is confined to a single region the structure may localize or trap light in the vicinity of the defect ${ }^{2}$. Extending the defect to a line results in light that may be trapped to the line region and build what is known as photonic (phononic) band gap waveguides ${ }^{5}$. As in the case of semiconductor electronics, the defect present in the artificial crystal can be used as the backdrop for a number of photonic (phononic) devices ${ }^{6,7,8}$

Photonic crystals may present themselves as 1-D, 2-D and 3-D in periodicity ${ }^{1,2}$. The 1-D structure is the typical Bragg grating, while the 2-D structure usually has periodicity in a flat plane $(\mathrm{x}, \mathrm{y})$ and uniform in the third direction $(\mathrm{z})$. The 3-D photonic crystal has periodicity in all three directions and is usually difficult to fabricate and requires a full vector wave analysis. In practical device configurations, the 2-D planar structure is suitable and the analysis can proceed in two ways; uniform and infinite in the third direction ${ }^{2}$; membrane with wave confined to the layer ${ }^{5}$. In such applications it is desirable to design devices that are as "polarization independent" as possible. In the optical domain this implies that the band gap for the two possible wave polarization states, transverse electric (TE) and transverse magnetic (TM) should overlap over the desired propagation frequencies and leads to the term "Full Optical Band Gap". Through a careful selection of the constituent materials making up the photonic crystal and choice of the crystal type (square, triangular, hexagonal), researchers have shown that a full optical band gap is possible in the 2-D structure and even in the 3-D structure ${ }^{9,10}$. Typically the lattice constant of the photonic crystals is on the scale of the wavelength of the light that will propagate through the 
medium. Phononic crystals are the acoustical analogue of the photonic crystals in which the mass density and speed of sound in the medium play the role of dielectric constant and speed of light. These may also be cast as 1-D, 2-D or 3-D structures. The lattice constant in the acoustical domain is also on the scale of the acoustical wavelength in the medium.

Since photonic crystals primarily depend on the dielectric constant of the constituent materials and the phononic crystal depend on the mass density and speed of sound in the constituent materials it is possible to design a single photonic / phononic lattice that contains both optical and acoustical band gaps simultaneously. Such a structure has been labelled the phoxonic crystal (PxC). Theoretical studies by Maldovan and Thomas recently have demonstrated the possibility for 2-D simultaneous bandgaps and localized states for optical and acoustic waves in a defect containing $\mathrm{PxC}$ structures ${ }^{11,12}$. Simultaneous optical-acoustic bandgaps in PxCs incorporating Lithium Niobate $\left(\mathrm{LiNbO}_{3}\right)$ have also been reported by SadatSaleh et. al. ${ }^{13}$. PxCs allow for enhanced optical-acoustic interaction ${ }^{14}$. Such effects have been reported in 1-D and 3-D PxC devices ${ }^{15,16}$.

\subsection{Motivation}

The goal of this thesis is to find a PxC structure that simultaneously exhibits both a complete optical band gap and an acoustical band gap for solid-solid materials (low contrast for both the optical and acoustic materials' properties) and to configure waveguides into such a structure. Instead of varying the lattice pattern or employing different inclusions size in the unit cell to widen optical and acoustical band gaps, multiple different inclusion shapes are used in this research.

An ongoing goal of phoxonic crystal research is the desire to obtain a complete optical and acoustical band gap at the same time within the same material constituents and lattice 
arrangement. Having a complete optical band gap for both polarization TE and TM will maximize the performance of PxCs by reducing the losses from defect states for any polarization. However, obtaining this condition has proven challenging. In addition, the low contrast for both the optical and acoustic materials' properties makes opening the simultaneously band gap unsuitable for some practical applications ${ }^{13}$. The majority of research on this problem has involved the use of symmetry-reduction techniques such as the introduction of different size of cylindrical inclusions into $\mathrm{PtC} / \mathrm{PnC}$ crystals ${ }^{9,10,17,18}$.

A different method is needed for modifying the Phoxonic crystal geometry. To the author's knowledge, no attempt has yet been made to utilize different inclusion shapes (ie. both high-dielectric rods and low-dielectric rod) in the same PxC structure in order to simultaneously expand the material's optical and acoustic band gaps for solid- solid materials.

\subsection{Contributions}

In this work a complete optical band gap and an acoustic band gap were obtained simultaneously for low contrast refractive index composite consisting of silicon and polymer (solid-solid structure). The proposed unit cell consisted of a combination of high dielectric inclusions and low dielectric inclusions which provide a reciprocal nature to the fields. In addition, using a line defect in the PxC structure was introduced and both optical and acoustic waves can be guided in the waveguide region through the band gap effect.

\subsection{Applications}

Giving that simultaneously optical and acoustic band gaps and waveguide, this $\mathrm{PxC}$ structure can be used in a number of acousto-optic interaction applications such as switches, tunable filter, or Y-branch splitter to name a few. The close proximity of the optical and acoustical fields in the waveguide promises to enhance the interaction and improve device performance. 


\section{Backgrounds}

\subsection{Introduction}

In this chapter the fundamental ideas which govern the wave properties of photonic and phononic crystals are presented. The master equations are retained until chapter 3 where a full theoretical development of the eigenvalue equations, suitable for band structure determination, will be provided. A historical account of significant developments in photonic, phononic and phoxonic technologies is included and places this research into context. The last few years has seen a boom in phoxonic research which underscores the great promise this technology holds $^{11,12,13,15,16}$.

\subsection{Basic Wave Properties Governing Photonic and Phononic Crystals}

Photonic and phononic crystals in their most popular form are one, two, or three-dimensional periodic structures composed of two or more materials with different optical or acoustic properties $^{1,2,4}$. Typically one of the materials dominates the structure and becomes the host material and provides mechanical support while the second material fills in voids patterned into the host. The photonic (phononic) crystal family also contains structures known as aperiodic crystals, quasi-crystals $^{19}$, and disordered crystals $^{20}$. Although these are interesting and have many unique properties they are not examined in this review or considered as candidate structures for the phoxonic research presented. Depending on the choice of materials and the geometry of the crystal structure, such structures may exhibit band gaps in there band structure, frequency ranges over which optical or acoustic waves cannot propagate through the structure. It is worth noting here that the optical and acoustical waves would normally be propagated in each of the pure constituent materials taken separately. 
The photonic crystal's optical properties are determined by the materials dielectric constant and geometrical arrangement. The 1-D structure composed of repeating layers of two materials shown in figure 2.1, was first proposed and described by Lord Rayleigh ${ }^{21}$ in 1887. However, the first modern research on photonic crystals was conducted by Eli Yablonovitch ${ }^{1}$ in 1987 who proposed that patterning the dielectric could take place in all three directions, as shown in figure 2.2. Yablonivitch proposed that the inhibition of spontaneous emission in photonic crystal band gaps could be utilized to significantly improve the performance of semiconductor lasers. In the same year and independently, S. John suggested the possibility of Anderson localization via the use of weak disorder in perfect photonic $\operatorname{crystals}^{20}$. Many applications of photonic crystals rely on the presence of large and complete band gaps, as reflectivity is the main requirement for devices such as filters, cavities or waveguides. Therefore, a significant component of photonic crystal research is the desire to design structures that contain a large photonic band gap for 3-D structures and when possible a large full photonic band gap for the two polarizations in 2-D planar structures ${ }^{9,10,22,23}$. In general, there are two main challenges in photonic crystal fabrication: the fabrication of dielectric structures with 3-D band gaps, and the fabrication of photonic crystals for high (optical) frequency ranges. Micro-fabrication and micromachining technologies offer one solution. One method for building two or threedimensional photonic crystals is electron-beam (e-beam) lithography. In this method, an electron beam writes the photonic pattern onto a sheet of photoresist material. The photoresist is then used as a mask for a matrix material, which is subsequently etched using a reactive ion etching (RIE) process. This process etches away the unmasked areas, transferring the photonic pattern to the matrix and creating a two-dimensional photonic crystal of air inclusions ${ }^{24,25,26}$. Another approach is to use interference lithography to build the photonic crystal and requires several laser 
beams which interfere in a photosensitive material producing a template for defining periodic structures in two dimensions ${ }^{27}$.

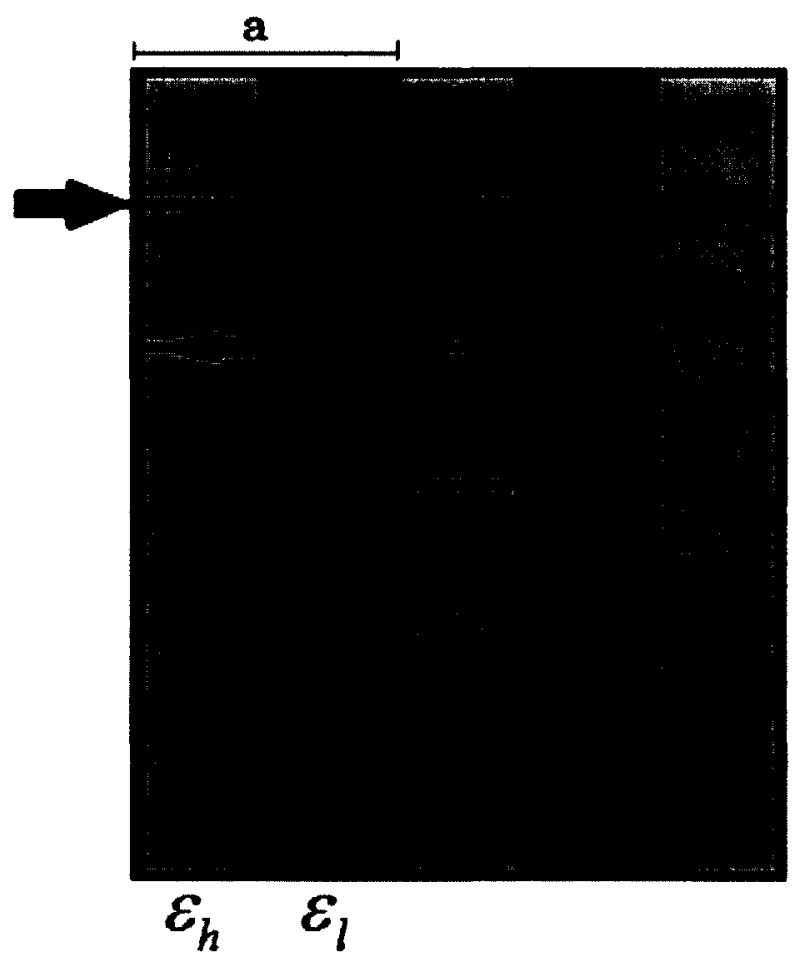

Figure 2.1: Constructive and destructive interferences in the forward and backward propagation directions, for one direction photonic crystals. $\varepsilon_{h}, \varepsilon_{l}$, are the high and low dielectric layers, respectively. 

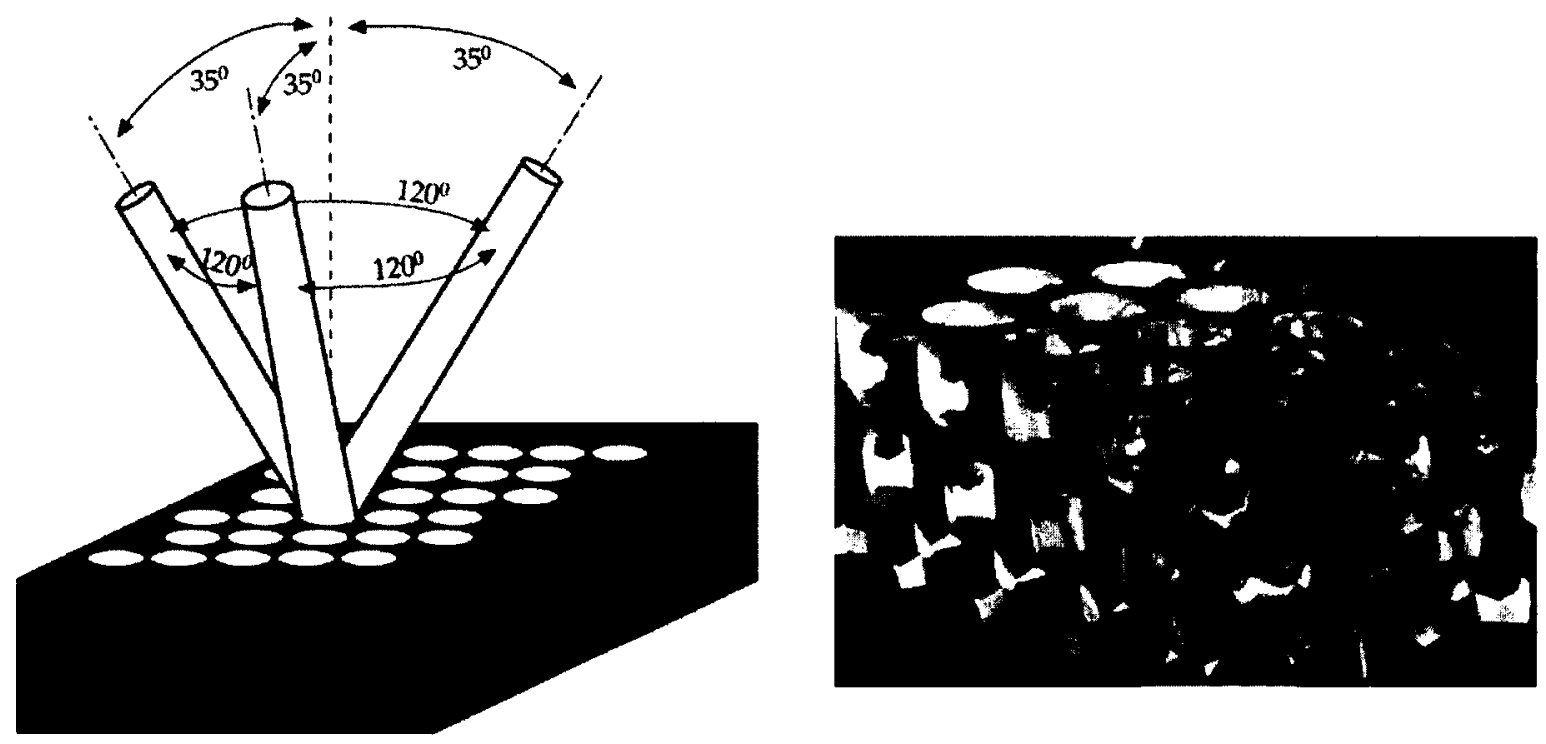

Figure 2.2: Yablonovite structure, reprinting with permission ${ }^{2}$; left: show how the Yablonovite structure is fabricated, right: Image Yablonovite structure using computer simulation.

Conceptually the band gaps observe in the transmission spectrums of photonic crystals result from the wave undergoing constructive and destructive interference as it propagates through the periodic medium. Taking the structure shown in figure 2.1 for instance, the incident wave is partially reflected and partially transmitted as it passes through each dielectric interface. Multiple reflections also take place resulting in a number of waves proceeding in the forward as well as the backwards direction. The phase relationship between all the forward propagating waves is determined by the thickness and dielectric constant of the individual layers. Constructive interference and the generation of a strong transmitted wave will result when the combined phase change after travelling through one grating period is an integer multiple of $(2 \pi)$. A strong back reflected wave would result when the grating period thickness and material dielectric values produce waves in phase, multiple integer of $2 \pi$, which includes a $\pi$ phase 
change when the reflection takes place on the low dielectric side of the interface ${ }^{2,5}$. The band gap location is associated with the specific wavelengths (frequencies) which are strongly back reflected by the periodic structure. As will be shown in the next chapter, when generating band diagrams, it is assumed that the periodic structure is infinite in extent and within the band gap regions no transmission is predicted. The governing equations for constructive and destructive interferences in the forward and backward propagation directions are given in equations (2.1):

$$
\begin{gathered}
d_{1} n_{1}+d_{2} n_{2}=\frac{m \lambda}{2} \\
d_{1} n_{1}+d_{2} n_{2}=(m-1 / 2) \frac{\lambda}{2}
\end{gathered}
$$

These equations indicate that by selecting the individual layer thicknesses, material dielectric constants, the wavelengths associated to the transmitted and back reflected waves can be tuned. The band diagram for a 1-D photonic crystals is shown in figure 2.3 and is produced using high-dielectric-constant material $\left(\varepsilon_{h}=13\right)$ of width $0.2 a$ and low-dielectric-constant material $\left(\varepsilon_{h}=1\right)$ of width $0.8 a$, with $a$ the grating period ${ }^{2}$. On the y axis is plotted the normalized frequency $w a / 2 \pi c$ with $c$ the speed of light. On the $\mathrm{x}$ axis is plotted the normalized $\mathrm{k}$ vector, $k a / 2 \pi$, over the irreducible Brillouin zone. The dark lines (band lines) indicate the relationship that exists between frequency of the wave and $\vec{k}$ vector for transmitted waves. The greyed out bands in the diagram indicate frequency regions that have no corresponding $\vec{k}$ vector and constitute the band gap regions for the 1-D structures with these parameters. The band diagram 
illustrates another important aspect in band gap engineering. The fact that the $\mathrm{x}$ and $\mathrm{y}$ axis can be represented normalized to the lattice constant indicates that the wavelength (frequency) band of the bandgaps scales with the lattice constant. A structure that is designed at one particular wavelength band can be shifted to another wavelength band by scaling the lattice constant with respect to the scaling of the wavelength.

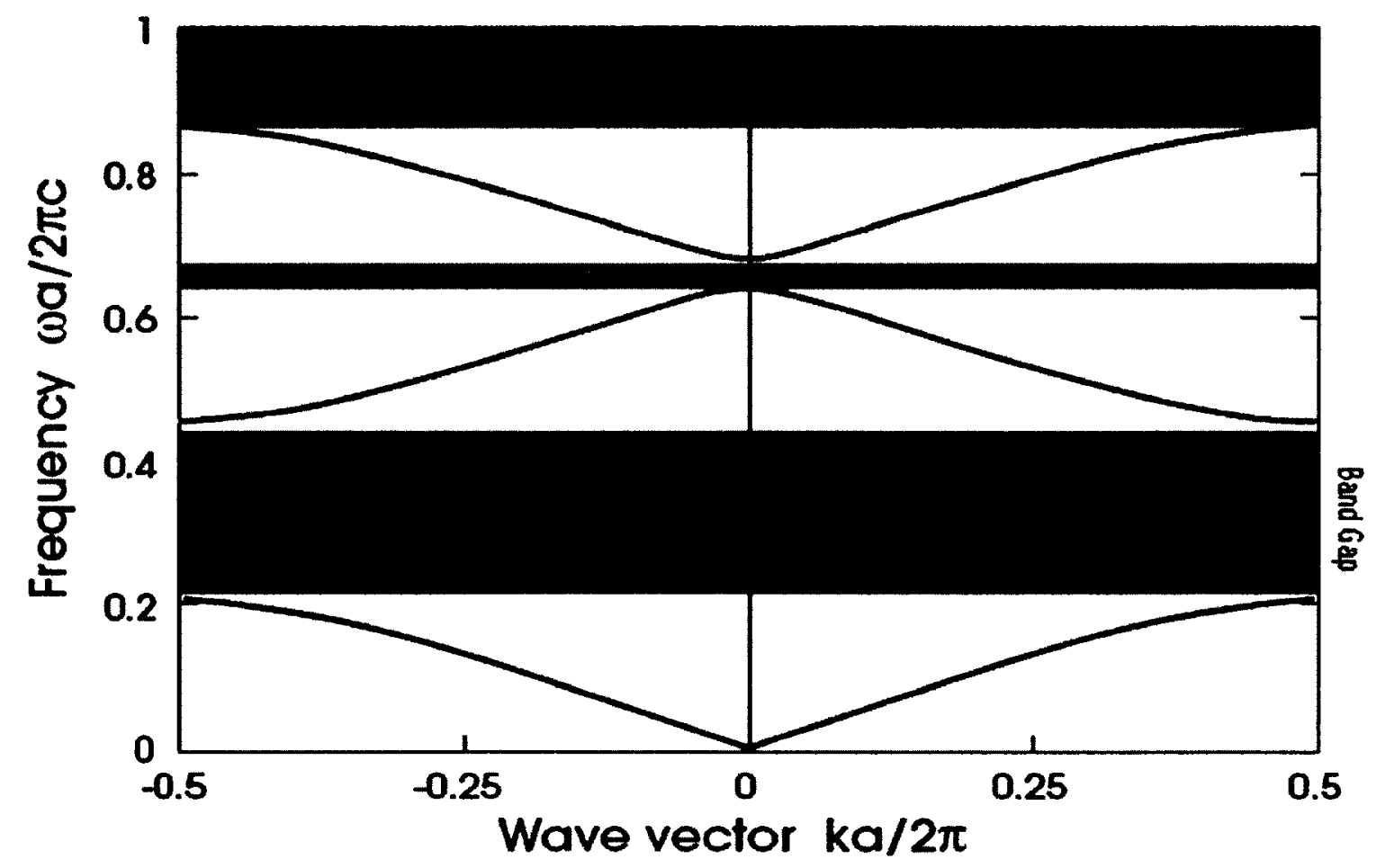

Figure 2.3: Band gap in a peridoic material with high-dielectric-constant material $\left(\varepsilon_{h}=13\right)$ of width $0.2 \mathrm{a}$ and low-dielectric-constant material $\left(\varepsilon_{h}=1\right)$ of width $0.8 \mathrm{a}$. Reprinted with permission ${ }^{2}$.

An alternate interpretation of the presence of bandgaps is provided via the variation theorem, which states that high-frequency wave will concentrate their energy in low-dielectricconstant regions while low-frequency wave will concentrate their energy in high-dielectric constant regions ${ }^{2}$. It can be noted that when the gap lies at symmetry points $\vec{k}=\pi / a$, the wave 24 | Chapter 2: Background 
has a wavelength of $2 a$. This means the nodes of this wave must be concentrated in the low or high-dielectric regions; otherwise the symmetry of the unit cell about its centre would be violated. Therefore if a structure has a large dielectric contrast, it may exhibit one or more band gaps.

A phononic crystal relies on a periodic arrangement of the mass density and speeds of sound in the constituent materials ${ }^{4}$. In a simple model, the entire structure can be regarded as individual mass elements interconnected through springs ${ }^{28}$. A periodic displacement of a mass element at one end of the crystal will introduce periodic forces on the neighbouring masses and under the right conditions a wave like displacement in the masses positions may be observed to propagate the length of the structure. In a manner similar to photonic crystal wave propagation, phononic waves can undergo the process of reflection and refraction. Phononic crystals, properly engineered can be designed to contain band gaps in their transmission spectrum as well. However, for acoustic waves propagating through an isotropic periodic structure there are two wave propagation mechanisms: mixed in plane displacement and transverse displacement ${ }^{28}$. The mixed mode has the wave propagation in the same direction as the displacement, while the transverse mode has the wave propagation perpendicular to the displacement direction. The band diagram returned in the acoustical analysis resembles to a large extent that shown in figure 2.3, for a 1-D phononic crystal. The $\mathrm{y}$ axis is labelled as normalized frequency, $f a$, and the $\mathrm{x}$ axis represents the $\mathrm{k}$ vector for acoustical waves.

The propagation of high-frequency sound through crystals was first studied by Narayanamurti et al in 1979 using one-dimensional GaAs/AlGaAs crystals ${ }^{4}$. The theoretical foundations of crystal phononics were subsequently established in 1993 by Kushwah, who developed the first full band structure equations for periodic elastic composites ${ }^{29}$. In 1998, 
Montero de Espinosa made the first experimental observation of ultrasonic acoustic band gaps in periodic structure ${ }^{30}$, discovering a gap in the $1000-1120 \mathrm{kHz}$ range in an Aluminium plate with cylindrical Mercury inclusions.

Recently, improvements in micro-fabrication and micromachining technologies using similar approaches as that mentioned for photonic crystal have yielded phononic crystals for frequencies up to $3 \mathrm{GHz}^{26}$. Using the interference lithography to place air inclusions in an epoxy host hypersonic frequencies, $(1 \mathrm{GHz})$, two dimensions phononic crystal have been made ${ }^{31}$. Selfassembly of polystyrene nano-sphere inclusions in a glass substrate subsequently back filled with silicon oil matrix is one way of building three dimensions phononic crystals ${ }^{32}$.

\subsection{Phoxonic Structures}

A phoxonic crystal is an artificial medium which may be regarded as a combination of a photonic and phononic crystal. In phoxonic structures the material properties and period arrangement are selected such that band gaps exist simultaneously for electromagnetic waves and acoustical waves. The band gap width of optics and acoustic should be large enough to be of practical use and the band gap centres should be located at the design frequency. In the photonics case, the band diagram makes use of the normalized frequency $w a / 2 \pi c$ which may also be written in terms of the free space wavelength as $a / \lambda$. For an optics based device, the centre of the band gap would be selected to correspond to the design wavelength in the telecommunications range $(\lambda=1550 \mathrm{~nm})$. Based on the design wavelength and optical band diagram, the lattice constant, $a$, is determined. This same lattice constant, in combination with the centre of the acoustical band gap can be used to determine the design acoustical frequency 
through, $f a$, equal to the mid-gap value. In this document, the centre of the normalized optical bandgap will be labelled NOBG and the centre of the acoustical band gap is labelled NABG.

As phoxonics combines acoustics and optics, the practical challenges involved are more complex. Among these challenges is that the available technologies for acoustic transducer work for the acoustic frequencies in the range of $1-3 \mathrm{GHz}$. On other hand, the nanoscale fabrication techniques required a good range of separation between any different two high or low materials of inclusions. For a specific wavelength, such as the telecommunication wavelength, these challenges required high frequency band gap level for optics and low frequency band gap level for acoustic. As mention previously, from a manufacturing standpoint a larger lattice size is preferable (especially for complex structures); therefore high-level optical band gaps are desirable. With current manufacturing techniques it is easier to produce low-acoustic-frequency transducers; therefore, larger lattices and lower NABG are preferable. Thus, the lattice requirements for both optical and acoustic wave propagation can be simultaneously obtained in the same material.

Acousto-optical interaction is the diffraction of light by acoustic waves. Acoustic waves propagating through a material induce mechanical strain that can change the material's local refractive index. The propagation of light through the material can potentially be controlled using sound. This effect has found a wide range of applications in devices such as modulators, tuneable filters and beam deflectors. Strong localization of both acoustic and electromagnetic waves can localize the acousto-optical interaction when waveguides within phoxonic crystal are available $^{33,34,35}$. 


\subsection{Recent Developments in Phoxonics}

Maldovan and Thomas " presented the notion of the 'deaf and blind' photonic-phononic crystals which contained simultaneous elastic and electromagnetic band gaps. The structures and band gap maps are shown in figure 2.4 for the square array, left column of images, and triangular array, right column of images. Band gap maps are produced by computing the band structure and then collecting the band gap information as a function of one or more of the structures parameters. The top row of figures represents the photonic band gap maps, for both TE and TM polarizations, and the lower row of figures represents the phononic band gap maps. These crystals consist of a periodic lattice of solid (silicon-red) and gas (air holes-white) elements. This solid-gas composition was chosen to permit simultaneous photonic/phononic band gaps, as photonic band gaps were rarely found in solid-solid structures ${ }^{2}$. Such crystals can consist either of solid inclusions in a gaseous matrix or gaseous inclusions (holes/gaps) in a solid matrix. The latter structure more reliably exhibits simultaneous photonic/phononic band gaps, the width of both gaps increasing as the solid volume fraction decreases. In a solid inclusion/gaseous matrix structure the photonic and phononic band gaps respond oppositely to the solid mass density, with the photonic band gap increasing with reduced density for TM, while no band gap for the TE polarization was observed. The best parameter were when the $r / a$ is around 0.45 for triangle lattice structures. This structure shows a complete optical and acoustical band gap, see figure 2.4. 

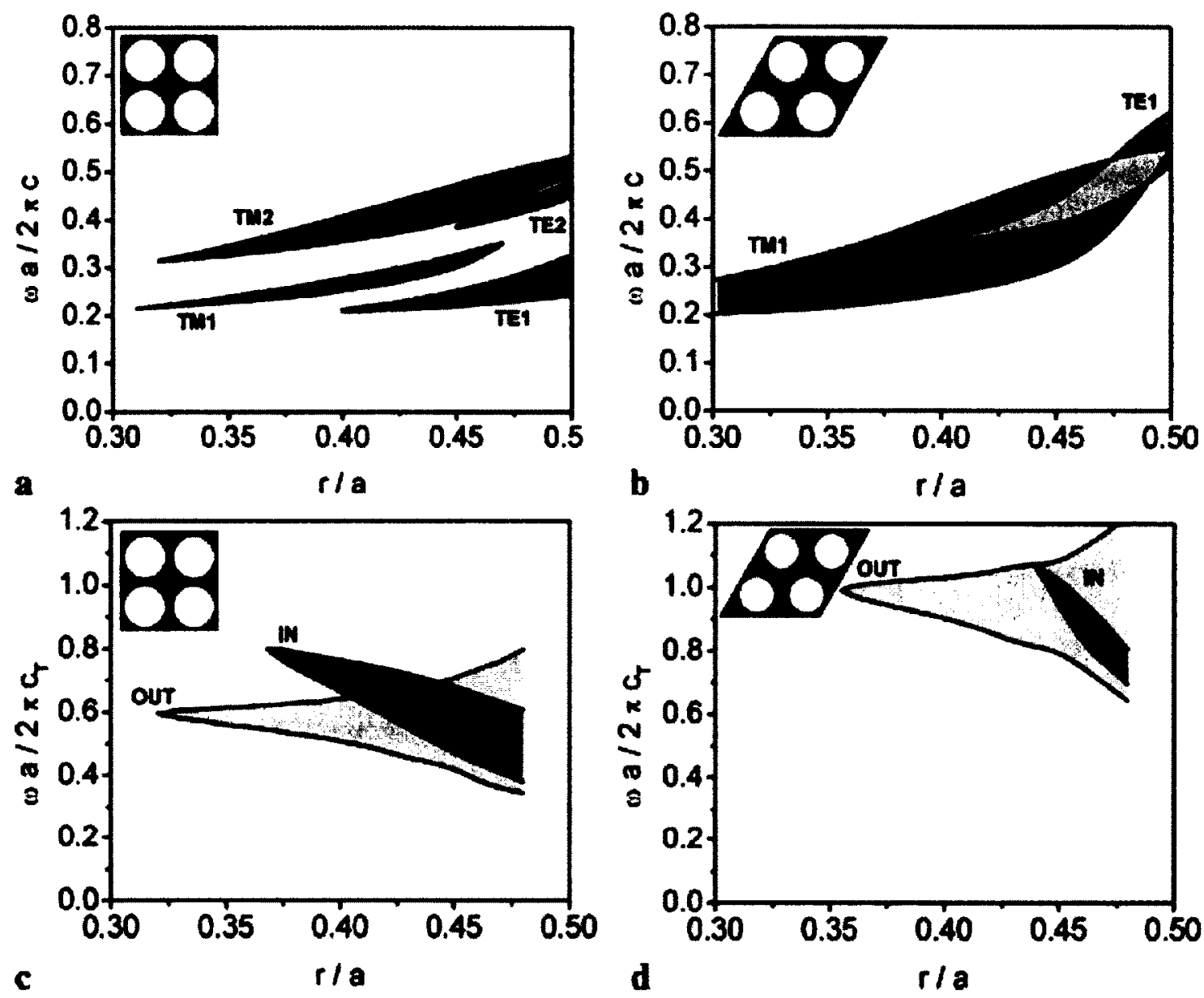

Figure 2.4: A triangular lattice of air inclusions in a silicon matrix, which exhibits complete phoxonic band gaps when $r / a$ is around 0.45 . Reprinted with permission ${ }^{11}$.

Phononic band gaps are easily obtained in both solid-solid ${ }^{36,37}$ and solid-gas structures ${ }^{38,39}$. A common technique for widening phononic band gaps is to decrease the symmetry of the crystal structure by, for example, adding atoms of different sizes in the unit cells ${ }^{9,10,17,18}$. That will reduce the symmetry since the number of angles that gives different appearance of the unit cell and the structure will increased. Sadat-Saleh et. $\mathrm{al}^{13}$ have investigated theoretically the creation of phoxonic band gaps in a Lithium Niobate matrix with air inclusions. Of the three lattice shapes investigated; single-atom square; single-atom hexagonal; multipleatom hexagonal, a hexagonal unit cell incorporating three different atoms yielded the widest 
band gap. Full photonic band gaps have not yet been obtained in this material, however, due to the low refractive index of Lithium Niobate limiting the photonic gaps. According to Salah's publication, cylindrical inclusions produced larger band gaps than other inclusion shapes. The report does not state what other shapes the cylindrical inclusions were compared against, nor the effect of multiple inclusion shapes in a single lattice.

In order to obtain simultaneously localized acoustic and electromagnetic modes in $\mathrm{PxC}$ structures, it is necessary to obtain both acoustic and electromagnetic band gaps. The literature reveals that this is difficult to accomplish using the common technique of cylindrical inclusions, especially if the material contrast is low. It is thus necessary to investigate a variety of inclusions of in the unit cell of the crystal structure. 


\section{Theories}

\subsection{Introduction}

In this chapter, the eigenvalue equations for the two optical polarizations and the transverse displacement of the acoustical waves are developed. The start point in the optical domain is Maxwell's equations simplified for the medium properties of interest and for the 2-D infinite geometry of the structure. The acoustical domain starts from Newton's second law for the same structure and planar geometry. The details of the plane wave method to solving for the band structure is detailed for the two wave types. The chapter also contains a discussion of the effect of having a defect present in the infinite periodic photonic and phononic crystal and the notion of a photonic crystal resonator and waveguide are discussed. The chapter concludes with a discussion of the acousto-optic effect, one of the methods in which the optical field may be influenced by an acoustical field.

\subsection{Maxwell's Equations}

Maxwell's equations are the fundamental equations of electromagnetic theory. In the derivation of the eigenvalue equations suitable for band structure computation, many simplifying assumptions are made. The mediums are assumed to have a real and frequency independent dielectric constant of relative permittivity $\varepsilon_{\mathrm{r}}$ and are also assumed to be non-magnetic with free space permeability $\mu_{0}$. The charge densities, and current density vector, are taken as zero. In summary, the mediums are treated as linear, lossless, non-magnetic and free source ${ }^{2}$. Maxwell's equations for an inhomogeneous material are as follows:

$$
\nabla \cdot \vec{H}(\vec{r}, t)=0
$$




$$
\begin{gathered}
\nabla \cdot[\varepsilon(\vec{r}) \vec{E}(\vec{r}, t)]=0 \\
\nabla \times \vec{E}(\vec{r}, t)+\mu_{o} \frac{\partial \vec{H}(\vec{r}, t)}{\partial t}=0 \\
\nabla \times \vec{H}(\vec{r}, t)-\varepsilon(\vec{r}) \frac{\partial \vec{E}(\vec{r}, t)}{\partial t}=0
\end{gathered}
$$

Where $\vec{E}$ is the electric field vector and $\vec{H}$ is the magnetic flux density vector. The curl expressions can be expanded in the $(x, y, z)$ Cartesian coordinate system, in which the electric field has components $\vec{E}=E_{x} \hat{x}+E_{y} \hat{y}+E_{z} \hat{z}$ and the magnetic field has the components $\vec{H}=H_{x} \hat{x}+H_{y} \hat{y}+H_{z} \hat{z}$. Starting with equation (3.3) the curl gives:

$$
\vec{\nabla} \times \vec{E}(\vec{r}, t)=\left|\begin{array}{ccc}
\hat{x} & \hat{y} & \hat{z} \\
\frac{\partial}{\partial x} & \frac{\partial}{\partial y} & \frac{\partial}{\partial z} \\
E_{x} & E_{y} & E_{z}
\end{array}\right|=\left(\frac{\partial E_{z}}{\partial y}-\frac{\partial E_{y}}{\partial z}\right) \hat{x}-\left(\frac{\partial E_{z}}{\partial x}-\frac{\partial E_{x}}{\partial z}\right) \hat{y}+\left(\frac{\partial E_{y}}{\partial x}-\frac{\partial E_{x}}{\partial y}\right) \hat{z}
$$

Inserting (3.5) into (3.3) gives a set of scalar equations relating the components of the electric and magnetic field:

$$
\begin{gathered}
\left(\frac{\partial E_{z}}{\partial y}-\frac{\partial E_{y}}{\partial z}\right)=-\mu_{0} \frac{\partial H_{x}}{\partial t} \\
\left(-\frac{\partial E_{z}}{\partial x}+\frac{\partial E_{x}}{\partial z}\right)=-\mu_{0} \frac{\partial H_{y}}{\partial t} \\
\left(\frac{\partial E_{y}}{\partial x}-\frac{\partial E_{x}}{\partial y}\right)=-\mu_{0} \frac{\partial H_{z}}{\partial t}
\end{gathered}
$$


The curl expression can also be applied to the magnetic field in equation (3.4).

$$
\vec{\nabla} \times \vec{H}=\left|\begin{array}{ccc}
\hat{x} & \hat{y} & \hat{z} \\
\frac{\partial}{\partial x} & \frac{\partial}{\partial y} & \frac{\partial}{\partial z} \\
H_{x} & H_{y} & H_{z}
\end{array}\right|=\left(\frac{\partial H_{z}}{\partial y}-\frac{\partial H_{y}}{\partial z}\right) \hat{x}-\left(\frac{\partial H_{z}}{\partial x}-\frac{\partial H_{x}}{\partial z}\right) \hat{y}+\left(\frac{\partial H_{y}}{\partial x}-\frac{\partial H_{x}}{\partial y}\right) \hat{z}
$$

When inserted into equation (3.4) results in the following three expressions relating the field components:

$$
\begin{aligned}
& \left(\frac{\partial H_{z}}{\partial y}-\frac{\partial H_{y}}{\partial z}\right)=\varepsilon(\vec{r}) \frac{\partial E_{x}}{\partial t} \\
& \left(-\frac{\partial H_{z}}{\partial x}+\frac{\partial H_{x}}{\partial z}\right)=\varepsilon(\vec{r}) \frac{\partial E_{y}}{\partial t} \\
& \left(\frac{\partial H_{y}}{\partial x}-\frac{\partial H_{x}}{\partial y}\right)=\varepsilon(\vec{r}) \frac{\partial E_{z}}{\partial t}
\end{aligned}
$$

For a full 3-D analysis the 6 field components are coupled. However, for two dimensions analysis in $(\mathrm{x}, \mathrm{y})$ and uniform infinite in z-direction, the field components decouple into two sets, one for TM and one for TE. The next section will examine the two dimensions case with the two decoupled sets of equations.

\subsection{TM and TE Wave Equations in the 2-D Photonic Crystal}

The typical 2-D planar, uniform and infinite in the $\mathrm{z}$ direction structures are shown in figure 3.1. The left side shows the square array and the right side shows the triangular array in the $(x, y)$ plane. The black region represents the background material which we will take as silicon, $\varepsilon_{\mathrm{Si}}$, and the white circular regions represent cylindrical holes drilled into the silicon which may be 
air, $\varepsilon_{\text {air }}$, or as presented latter a polymer, $\varepsilon_{\text {polymer. }}$ The holes axis runs parallel to the $z$ coordinate axis. As indicated above, for this particular geometry the 6 field components in equations (3.6), (3.7), (3.8), (3.10), (3.11), and (3.12) separate into two sets. One set contains only the $\left(E_{z}\right)$ field component with $\left(H_{x}, H_{y}\right)$ in the plane. This triplet is called the transverse magnetic (TM) polarization. The other set contains only the $\left(H_{z}\right)$ field component with $\left(E_{x}, E_{y}\right)$ in the plane. This triplet is called the transverse electric (TE) polarization. Since the two polarizations are presented by equations which are independent of the other polarization's field components, the TM and TE polarizations can be treated independently of each other. This leads to separate band structure expressions for each polarization and are much simpler to solve that trying to work in full 3-D for an arbitrary 3-D photonic crystal.

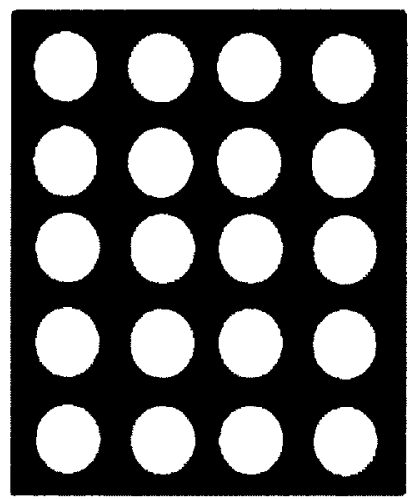

Square Lattice

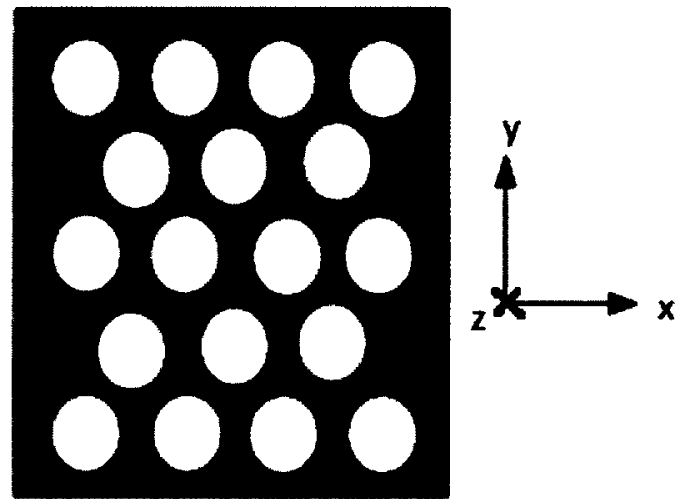

Triangle Lattice

Figure 3.1: Square (left) and triangle (right) lattice photonic crystals. Black and white represents different materials used in the crystals. 


\subsubsection{TM Polarization}

For the geometry and coordinate system shown in figure 3.1 , the TM polarization is characterized with the electric field parallel to the rods axis (in the z-direction). The electric field components are $\left(E_{x}=0, E_{y}=0, E_{z}\right)$. For a uniform and infinite medium in the $\mathrm{z}$ direction, derivatives of any field component with respect to $\mathrm{z}$ can be set to zero, $\frac{\partial}{\partial z} \rightarrow 0$. Based on these, equations (3.6), (3.7) and (3.12) can be isolated from the original 6 equations and can be rewritten as:

$$
\begin{gathered}
\left(\frac{\partial E_{z}}{\partial y}\right)=-\mu_{0} \frac{\partial H_{x}}{\partial t} \\
\left(-\frac{\partial E_{z}}{\partial x}\right)=-\mu_{0} \frac{\partial H_{y}}{\partial t} \\
\left(\frac{\partial H_{y}}{\partial x}-\frac{\partial H_{x}}{\partial y}\right)=\varepsilon(\vec{r}) \frac{\partial E_{z}}{\partial t}
\end{gathered}
$$

Taking the time derivatives of equation (3.15) gives:

$$
\left(\frac{\partial}{\partial t} \frac{\partial H_{y}}{\partial x}-\frac{\partial}{\partial t} \frac{\partial H_{x}}{\partial y}\right)=\varepsilon(\vec{r}) \frac{\partial^{2} E_{z}}{\partial t^{2}}
$$

Taking the y-derivative of equation (3.13)

$$
\left(\frac{\partial^{2} E_{z}}{\partial y^{2}}\right)=-\mu_{0} \frac{\partial}{\partial y} \frac{\partial H_{x}}{\partial t}
$$

Taking the $\mathrm{x}$-derivative of equation (3.14) 


$$
\left(-\frac{\partial^{2} E_{z}}{\partial x^{2}}\right)=-\mu_{0} \frac{\partial}{\partial x} \frac{\partial H_{y}}{\partial t}
$$

By combining equations (3.16), (3.17) and (3.18), and recognizing the temporal and special derivative order may be interchanged gives the wave equation for the $E_{z}$ field component:

$$
\frac{1}{\varepsilon_{r}(\vec{r})}\left(\frac{\partial^{2} E_{z}}{\partial x^{2}}+\frac{\partial^{2} E_{z}}{\partial y^{2}}\right)=\frac{1}{c^{2}} \frac{\partial^{2} E_{z}}{\partial t^{2}}
$$

Once the $E_{z}$ component is solved using equation (3.19), the field components $\left(H_{x}, H_{y}\right)$ can be solved using equation (3.13) and (3.14).

\subsubsection{TE Polarization}

For the geometry and coordinate system shown in figure 3.1, the TE polarization is characterized with the magnetic field parallel to the rods axis (in the $\mathrm{z}$-direction). The magnetic field components are $\left(H_{x}=0, H_{y}=0, H_{z}\right)$. For a uniform and infinite medium in the $\mathrm{z}$ direction, derivatives of any field component with respect to $\mathrm{z}$ can be set to zero, $\frac{\partial}{\partial z} \rightarrow 0$. Based on these, equations (3.8), (3.10) and (3.11) can be isolated from the original 6 equations and can be rewritten as:

$$
\begin{gathered}
\left(\frac{\partial E_{y}}{\partial x}-\frac{\partial E_{x}}{\partial y}\right)=-\mu_{o} \frac{\partial H_{z}}{\partial t} \\
\left(\frac{\partial H_{z}}{\partial y}\right)=\varepsilon(\bar{r}) \frac{\partial E_{x}}{\partial t}
\end{gathered}
$$




$$
\left(-\frac{\partial H_{z}}{\partial x}\right)=\varepsilon(\vec{r}) \frac{\partial E_{y}}{\partial t}
$$

Equation (3.21) and (3.22) can be written as:

$$
\begin{gathered}
\frac{1}{\mathcal{E}(\bar{r})}\left(\frac{\partial H_{z}}{\partial y}\right)=\frac{\partial E_{x}}{\partial t} \\
\frac{1}{\varepsilon(\vec{r})}\left(-\frac{\partial H_{z}}{\partial x}\right)=\frac{\partial E_{y}}{\partial t}
\end{gathered}
$$

Taking the time derivatives of equation (3.20) gives,

$$
\left(\frac{\partial}{\partial t} \frac{\partial E_{y}}{\partial x}-\frac{\partial}{\partial t} \frac{\partial E_{x}}{\partial y}\right)=-\mu_{o} \frac{\partial^{2} H_{z}}{\partial t^{2}}
$$

Taking the y-derivative of equation (3.23) gives

$$
\frac{\partial}{\partial y}\left(\frac{1}{\varepsilon(\bar{r})}\left(\frac{\partial H_{z}}{\partial y}\right)\right)=\frac{\partial}{\partial y} \frac{\partial E_{x}}{\partial t}
$$

Taking the $\mathrm{x}$-derivative of equation (3.24)

$$
\frac{\partial}{\partial x}\left(\frac{1}{\varepsilon(\vec{r})}\left(-\frac{\partial H_{z}}{\partial x}\right)\right)=\frac{\partial}{\partial x} \frac{\partial E_{y}}{\partial t}
$$

By combining equations (3.25), (3.26) and (3.27), and recognizing the temporal and special derivative order may be interchanged gives the wave equation for the $H_{z}$ field component.

$$
\frac{\partial}{\partial x}\left(\frac{1}{\varepsilon_{r}(\vec{r})}\left(\frac{\partial H_{z}}{\partial x}\right)\right)+\frac{\partial}{\partial y}\left(\frac{1}{\varepsilon_{r}(\vec{r})}\left(\frac{\partial H_{z}}{\partial y}\right)=\frac{1}{c^{2}} \frac{\partial^{2} \vec{H}_{z}}{\partial t^{2}}\right.
$$


Once the $H_{z}$ component is found, the $\mathrm{E}$ components $\left(E_{x}, E_{y}\right)$ can be solved for using equations (3.21) and (3.22). In equations (3.19) and (3.28) the time dependence is of the form $e^{-j \omega t}$, therefore the time derivative term will give $-\omega^{2} e^{-j \omega t} E_{z}$. The plane wave approach presented later in this chapter is used to solve for the frequency $(\omega)$.

\subsection{The Plane Wave Method}

Plane wave method is a method that is usually preferred when studying the band structure of periodic structures. However, this method is based on the assumption of studying infinite structures. By using Fourier series to represent the medium and the Bolch Wave Theorem to represent the electromagnetic field propagating through it, then inserting them in the twodimensional equations (3.19) and (3.28), an eigenvalue matrix equation is obtained. Solving the matrix will give eigenvalues for the frequencies $\omega$, permitted to propagate in the medium. The eigenvectors are obtained by reinserting individual eigenvalues in the matrix.

\subsubsection{One Dimension Photonic Crystal}

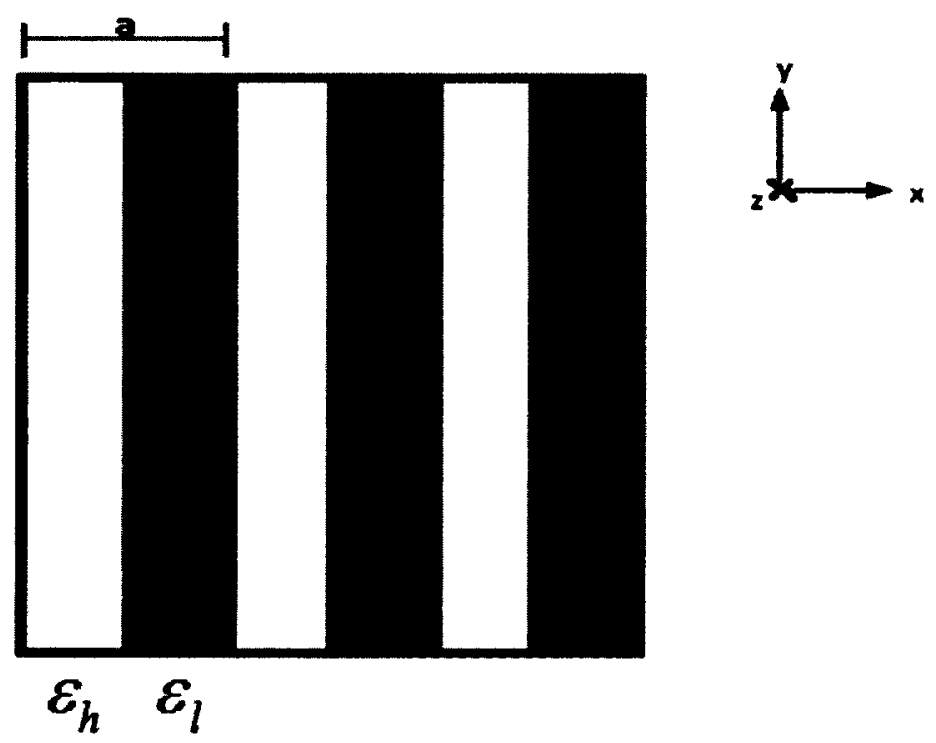


Figure 3.2: One dimension photonic structure. Black represent low dielectric and white represent high dielectric.

The one dimension photonic crystal, shown in figure 3.2 , is the easiest structure for studying and understanding the main properties of photonic crystal. For one dimension periodic structure, where the electric filed is parallel to the z-directions and to the layers, as shown in figure 3.2, Bloch solutions can be written as

$$
E_{z}(x, w)=\sum_{\bar{G}}^{\infty} K_{(\bar{k}, \bar{G})}^{E_{z}} e^{-j(\bar{k}+\bar{G}) x} e^{-j w t}
$$

where $\vec{G}=2 \pi / a$ is the reciprocal lattice, and $\vec{k}=2 \pi / \lambda \hat{x}$. These solutions are periodic with $\vec{k}$, the solutions of $\vec{k}$ is identical to the solutions of $\vec{k}+(2 \pi p / a) \hat{x}$, where $(p= \pm 0, \pm 1, \pm 2, \ldots)$. Since the solutions are identical for the set of wave numbers $\vec{k}, \vec{k}+(2 \pi p / a \hat{x}), \ldots$ all possible normal modes of the matrix equation can be obtained by letting $k$ vary from $-\pi / a$ to $\pi / a$ (the Brillouin zone for 1-D periodic system)

$$
\frac{-\pi}{a} \leq k \leq \frac{\pi}{a}
$$

Many of the properties employed in one-dimensional analysis can be applied in two or three dimensions. Solving the wave equations (3.19) and (3.28) with the additional notation that $\partial / \partial y \rightarrow 0$ gives a band structure similar to that in figure 2.3 .

\subsubsection{Two Dimensions Photonic Crystal}

Two dimension photonic crystals have the advantage of controlling the light in two directions. In addition, the two dimensions is easer in calculation than the three dimensions photonic crystals. 
These advantages and the fact that 2-D structures are easier to fabricate make it more suitable for applications.

For two dimensional square lattice photonic crystal, the Bravais lattice in the $(x, y)$ plane is shown figure 3.3. Its sites are given by $\bar{X} \vec{Y}_{I l}(l)=l_{1} \vec{a}_{1}+l_{2} \vec{a}_{2}$ where $l_{1}$ and $l_{2}$ are arbitrary integers and $\vec{a}_{1}, \vec{a}_{2}$ are the lattice vectors. The area of the unit cell of the lattice is given by:

$$
a_{c}=\left|\vec{a}_{1} \times \vec{a}_{2}\right|
$$

The dielectric constant of this system is a position-dependent function $\varepsilon\left(\bar{X} \vec{Y}_{/ \prime}\right)$ where:

$$
\bar{X}_{l /}=x \hat{x}+y \hat{y}
$$

Since the system is periodic, $\varepsilon\left(\bar{X} \bar{Y}_{\| l}\right)$ satisfies the relation

$$
\varepsilon(\bar{X} \vec{Y})=\varepsilon(\bar{X} \vec{Y}+\bar{X} \vec{Y}(l))
$$

Since equation (3.28) uses $1 / \varepsilon(\vec{r})$ then the inverse dielectric constant is represented using the Fourier series:

$$
\frac{1}{\varepsilon\left(\bar{X} \vec{Y}_{l l}\right)}=\sum_{\bar{G}_{l l}}^{\infty} K_{\bar{G}_{\| \prime}}^{\varepsilon} e^{-j \vec{G}_{\|} \bar{X}_{\| \prime}}
$$

With $K_{\bar{G}_{\| \prime}}^{\varepsilon}$ the expansion coefficients and $\bar{G}_{\| /}$the reciprocal lattice vectors. 

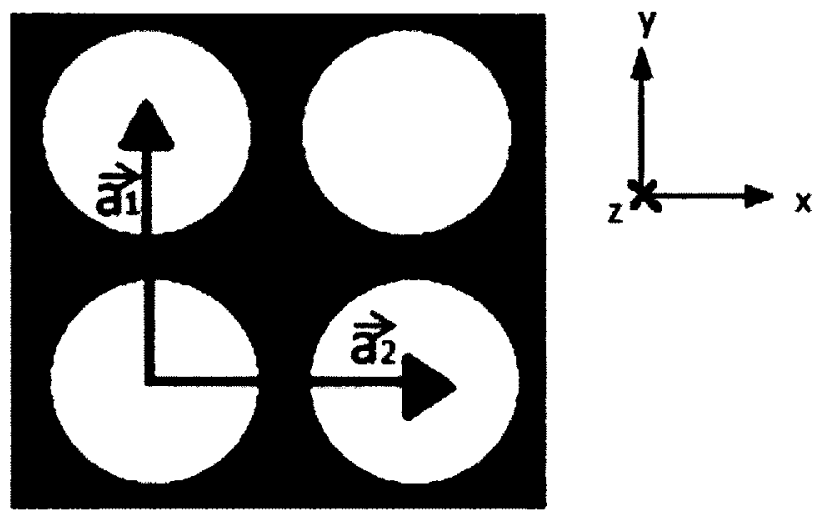

Figure 3.3: Bravais lattice for two dimensions square lattice photonic crystal. Black represent low dielectric and white represent high dielectric.

The Bloch solutions for two dimensions case considered here can be written as for TM and TE, respectively

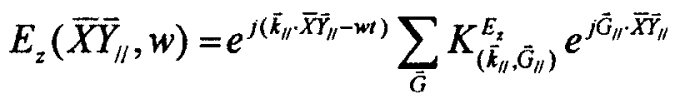

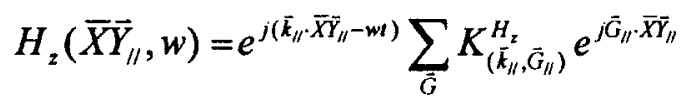

With $K_{\left(\bar{k}_{l}, \bar{G}_{l l}\right)}^{E_{z}}$ and $K_{\left(\bar{k}_{l}, \bar{G}_{l l}\right)}^{H_{z}}$ the expansion coefficients. Therefore, for two dimensions all possible normal modes for the equation can be found by letting the $\vec{k}$ vector vary from $\Gamma$ to $\mathrm{X}$ to $\mathrm{M}$ and back to $\Gamma$ along the straight lines in reciprocal space (see figure 3.4). 


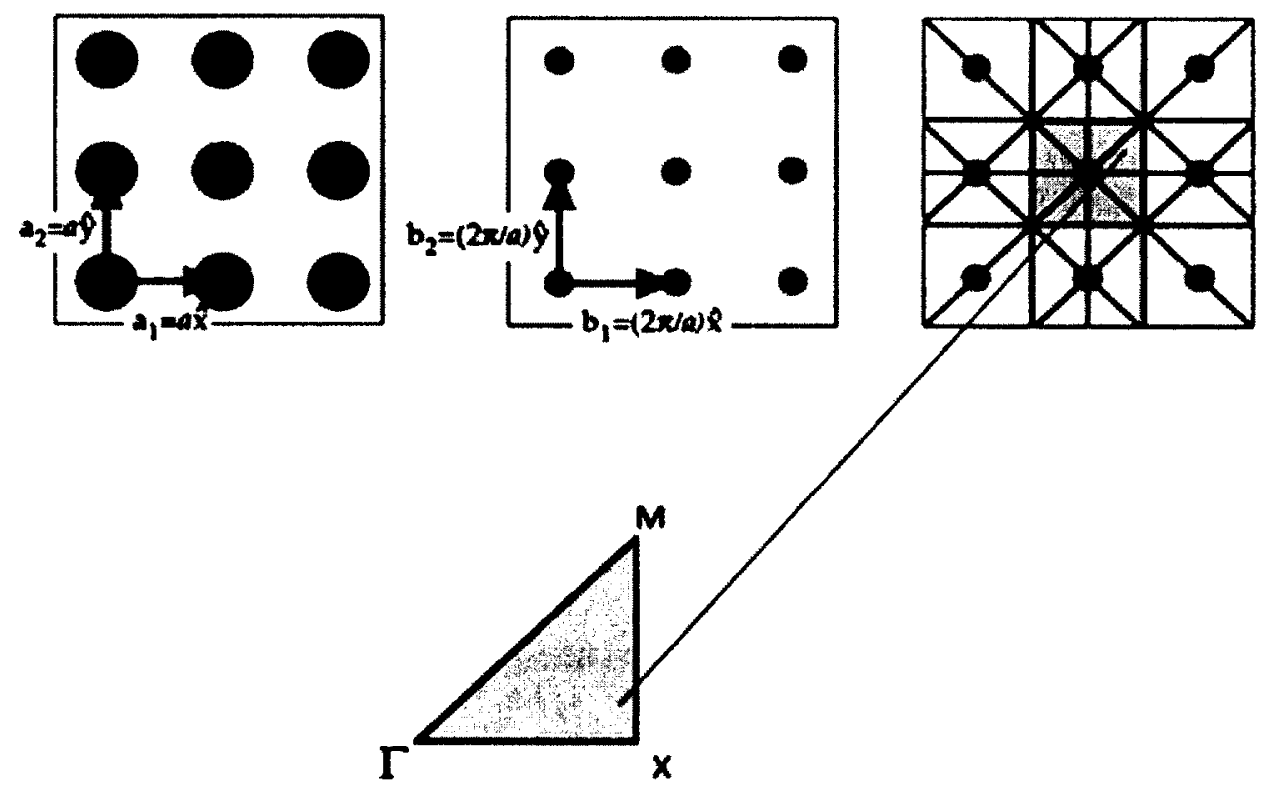

Figure 3.4: The symmetry points of the two dimensions square lattice ${ }^{2}$.

\subsubsection{TM Application}

For the TM-polarization, we obtain $\vec{E}$ and $\vec{H}$ in the following forms

$$
\begin{gathered}
\bar{E}(\vec{r}, t)=\left(0,0, E_{z}\left(\bar{X} \vec{Y}_{/ /}, w\right) e^{-j w t}\right. \\
\bar{H}(\vec{r}, t)=\left(H_{x}\left(\bar{X} \bar{Y}_{/ /}, w\right), H_{y}\left(\bar{X} \bar{Y}_{/ l}, w\right), 0\right) e^{-j w t}
\end{gathered}
$$

By substituting equations (3.34) and (3.35) into equations (3.19), multiplying both sides by $e^{-j G \cdot r}$ , integrating over the unit cell and making use of the orthogonalty properties of the expansion function, for nontrivial solutions the summation will be reduced (see appendix for the details in 
one dimension), allowing equation (3.19) to be written as an eigenvalue equation for the frequencies $\omega$ :

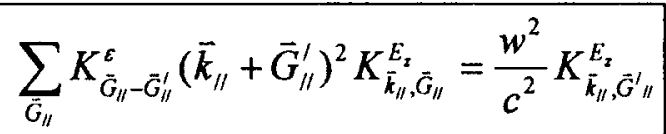

Where $\quad \vec{G}_{l l}=m \vec{b}_{1}+p \vec{b}_{2}, \quad \vec{k}_{l l}=\hat{x} k_{x}+\hat{y} k_{y}$, and the reciprocal lattice vectors $b_{1}$ and $b_{2}$ are defined by : $a_{i} \cdot b_{j}=2 \pi \delta_{i j}$ where $\delta_{i j}$ is the Kronecker delta symbol.

The equation (3.39) above can also be written as:

$$
\Rightarrow \sum_{m=-\infty}^{\infty} \sum_{p=-\infty}^{\infty} K_{n-m, q-p}^{\varepsilon_{r}} K_{m, p}^{E_{z}}\left[\left(\frac{2 \pi n}{a}+k_{x}\right)^{2}+\left(\frac{2 \pi q}{a}+k_{y}\right)^{2}\right]=\frac{w^{2}}{c^{2}} K_{m, p}^{E_{z}}
$$

Where the left side is a function of four variables that can be entered into an eigenvalue matrix, with $\mathrm{m}$ and $\mathrm{p}$ determining the columns and $\mathrm{n}$ and $\mathrm{q}$ determining the rows.

$$
\left[\begin{array}{cccc}
Q(m, p, n, q))_{m=-2, p=-2,(n=-2, \ldots+2, q=-2 \ldots+2)} & \cdots & \cdots & \cdots \\
\vdots & & & \\
\vdots & Q(m, p, n, q))_{m=2, p=2, n=2, q=2}
\end{array}\right]\left[\begin{array}{c}
K_{m=-2, p=-2}^{E_{z}} \\
K_{m=-2, p=-1}^{E_{z}} \\
\vdots \\
\vdots \\
K_{m=2, p=1}^{E_{z}} \\
K_{m=2, p=2}^{E_{z}}
\end{array}\right]=\frac{w^{2}}{c^{2}}\left[\begin{array}{c}
K_{m=-2, p=-2}^{E_{z}} \\
K_{m=-2, p=-1}^{E_{z}} \\
\vdots \\
\vdots \\
K_{m=2, p=1}^{E_{z}} \\
K_{m=2, p=2}^{E_{z}}
\end{array}\right]
$$

\subsubsection{TE Application}

For the TE polarization the $\bar{E}$ and $\bar{H}$ have the following form

$$
\vec{H}(\bar{r}, t)=\left(0,0, H_{z}\left(\bar{X} \bar{Y}_{/ /}, w\right) e^{-j w t}\right.
$$




$$
\vec{E}(\vec{r}, t)=\left(E_{x}\left(\bar{X} \vec{Y}_{/ l}, w\right), E_{y}\left(\bar{X} \vec{Y}_{\| /}, w\right), 0\right) e^{-j w t}
$$

By substituting equations (3.34) and (3.36) into equations (3.28), and using the same analysis as for TM polarization, the TE polarization eigenvalue equation will have the following form:

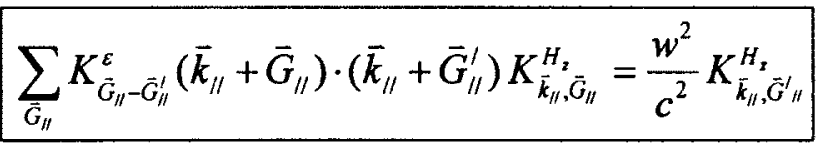

Using the plane wave expansion method the eigenvalue equations (3.39) and (3.43) are constructed. The matrix are populated using indices $(m, n, p, q)$ as outlined in equation (3.40a). These equations written into MATLAB will be used to generate the band structures for TM and TE optical modes respectively. In the next main section the transverse acoustic mode will be studied and the plane wave equation of that mode will be shown as well.

\subsubsection{MATLAB Programming of the Eigenvalue Equations}

In this work a MATLAB code have been created and used for all the calculations. Two main codes are presented in the Appendix B and C, for the optical and acoustic calculations, respectively. Each program has three parts. The first part is the discretising of the unit cell's. The second part is the Fourier transform of the unit cell's. The last part is the building and solving the eigenvalue matrix equation. As shown before, the method used for writing this code is plane wave method. This method is based on Fourier expansion of the unit cell, and the solutions are based on Bloch wave which are modulated by the periodicity of the structures. Therefore, the accuracy of the results depends on the number of the plane wave used. The results this code gives were checked with the results of some published papers and books ${ }^{2}$. For two dimensions photonic crystal structure for a square array of dielectrics columns with $r=0.2 a$, the dielectric parameters are given as $\varepsilon_{l}=1, \varepsilon_{h}=8.9$. The results band structure for TM and TE, shown in figure 3.5, respectively, were generated using my MATLAB program. The results match the result published in the reference [2]. 

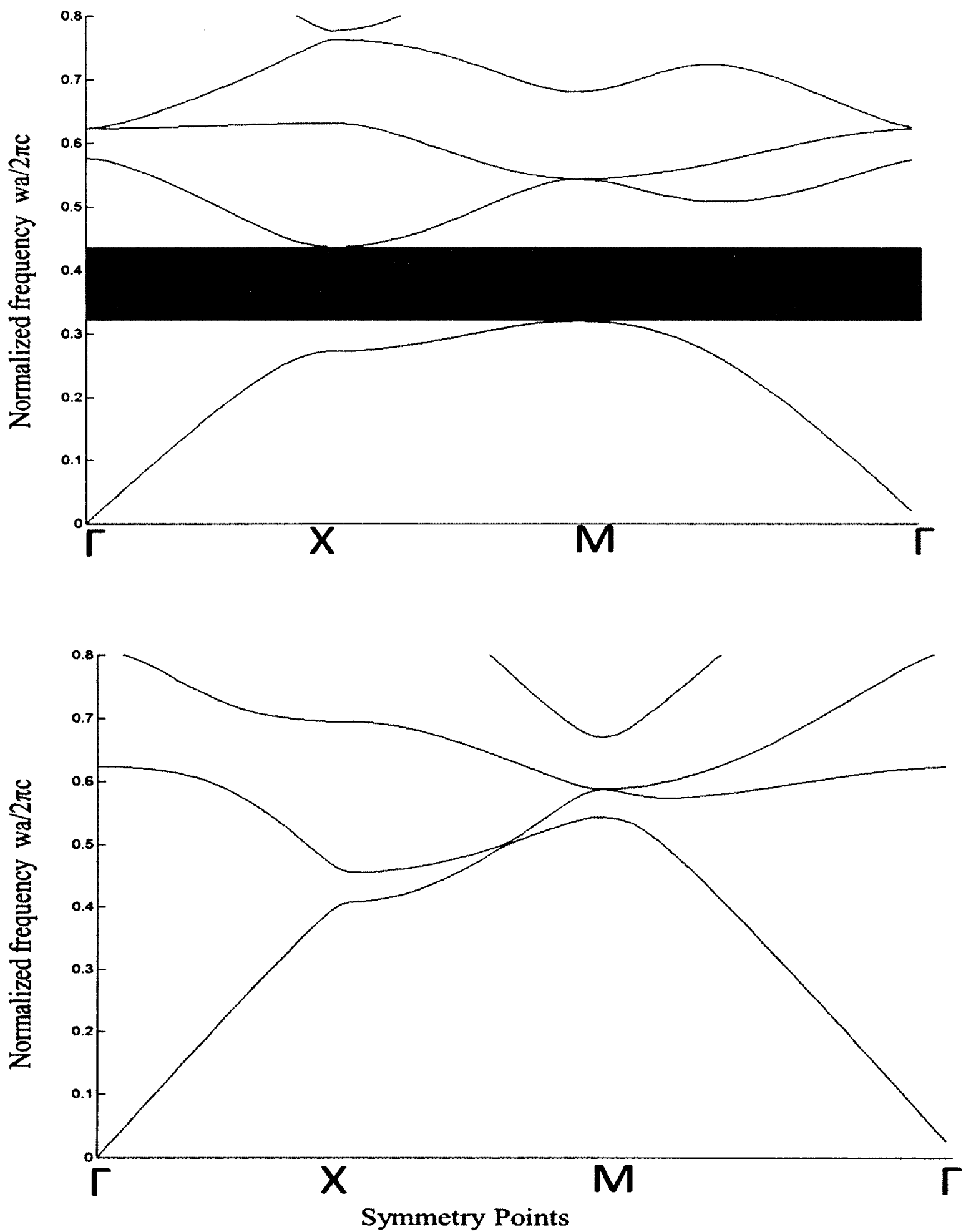

Figure 3.5: The band structure reproduce using my MATLAB code in Appendix B, for the same parameters as in (ref.2). (Top) :TM , (Bottom): TE 


\subsection{Acoustic Waves}

As mention before phononic crystal relies on a periodic arrangement of two different material that have two different acoustics properties (the mass density and speeds of sound) ${ }^{4}$. For formally analysis a simple quasi-one-dimensional structure of area (A) and of uniform elements $\mathrm{dx}$ will be considered, and $u(x, t)$ is the longitudinal displacement of these elements from their rest positions. The stress of this model can be written as ${ }^{28}$ :

$$
T=Y(x) e
$$

Where (e) is the strain $(e=d u / d x), \mathrm{Y}$ is Young's modulus, and $T(x)$ is the force per unit area A. Newton's second law can be used to describe the dynamics of the mass-containing element $\mathrm{dx}$ :

$$
\rho A d x \frac{\partial^{2} u(x, t)}{\partial t^{2}}=[T(x+d x)-T(x)] A
$$

Using Hooke's law, the right hand side of the equation (3.45), can be written as:

$$
T(x+d x)-T(x)=\frac{\partial T}{\partial x} d x=Y \frac{\partial^{2} u}{\partial x^{2}} d x
$$

By combining these two equations (3.45) and (3.46), the one dimensional equation is written as :

$$
\frac{\partial^{2} u}{\partial x^{2}}=\frac{\rho}{Y} \frac{\partial^{2} u}{\partial t^{2}}
$$

The generalization of this equation to three dimension and isotropic inhomogeneous medium, the Young's modulus $Y$ is replaced by the following matrix: 


$$
Y=c_{i j}=\left[\begin{array}{llllll}
c_{11} & c_{12} & c_{12} & 0 & 0 & 0 \\
c_{12} & c_{11} & c_{12} & 0 & 0 & 0 \\
c_{12} & c_{12} & c_{11} & 0 & 0 & 0 \\
0 & 0 & 0 & c_{44} & 0 & 0 \\
0 & 0 & 0 & 0 & c_{44} & 0 \\
0 & 0 & 0 & 0 & 0 & c_{44}
\end{array}\right]
$$

$c_{11}$ relates the compressive stress to the strain along the same direction, $c_{44}$ which relates the shear stress to the strain along the same direction and $c_{12}$ relates the compressive stress in one direction and the strain in another direction ${ }^{3}$. In addition, the mass density and the Young's modulus is position dependent due to the spatial distributions of the two constituents. In this work only the transverse mode is considered, thus the general wave equation reduces to $^{28}$ :

$$
\rho \frac{\partial^{2} \vec{u}_{z}(x, y, t)}{\partial t^{2}}=\nabla_{T} \cdot\left(\rho c_{t}^{2} \nabla_{T} \vec{u}_{z}(x, y, t)\right)
$$

The displacement in this equation is in the z-direction, which is perpendicular to the periodicity plane and parallel to the inclusions.

\subsubsection{Plane Wave Method for the Acoustic Wave}

When the structure has periodicity in $(\mathrm{x}, \mathrm{y})$ with two different materials present, the mass density and the longitude and transverse speed of sound are the $(x, y)$ plane dependent variables, $\rho\left(\bar{X}_{/ /}\right), c_{l}\left(\bar{X} \bar{Y}_{\| /}\right)$and $c_{t}\left(\bar{X}_{/ /}\right)$respectively and can be expanded using Fourier series ${ }^{40}$ :

$$
\begin{aligned}
& \rho\left(\bar{X} \bar{Y}_{\| /}\right)=\sum_{\bar{G}} K_{\bar{G}_{\| l}}^{\rho} e^{j \vec{G}_{\| \prime} \cdot \bar{X}_{Y^{\prime \prime}}} \\
& \rho c_{l}^{2}\left(\bar{X} \bar{Y}_{\| l}\right)=\sum_{\bar{G}} K_{\bar{G}_{l l}}^{\rho c_{l}^{2}} e^{j \bar{G}_{\| l} \cdot \bar{X}_{I^{\prime \prime}}}
\end{aligned}
$$




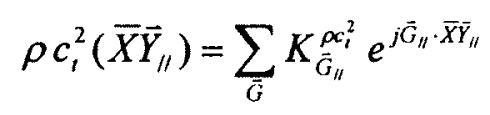

For such systems the Bloch theorem is satisfied for the displacement and may be expressed as :

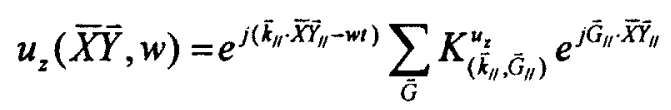

Where $K_{\left(\bar{k}_{u}, \bar{G}_{l l}\right)}^{u_{z}}$ are the expansion coefficients. By substituting equations (3.50), (3.52) and (3.53) into equation (3.49) then multiplying by the $e^{-j \vec{G} \vec{r}}$ and integrating over unit cell and using the orthogonally properties, the following equation is obtained:

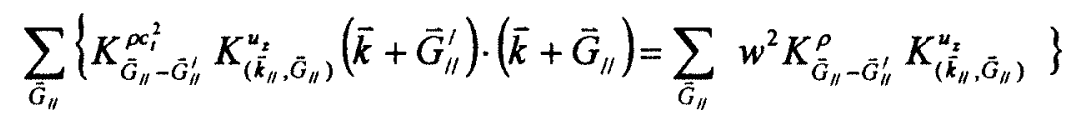

Equation (3.54), as written is not an eigenvalue equation due to the presence of the $K_{\bar{G}^{\prime \prime}-\bar{G}_{\| \prime}^{\prime \prime}}^{\rho}$ coefficients on the right hand side. The desired eigenvalue equations should have the following form:

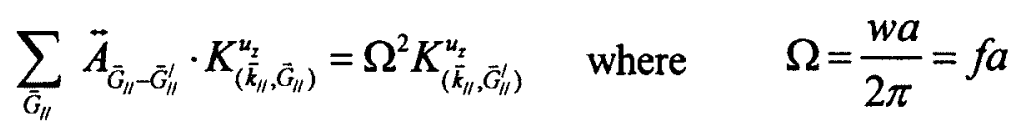

With this equation one can solve for each value of $k$ to give all solutions for ( $f a$ ), rather than solving for each value of $k$ to give each solution of $(f a)$.

Equation (3.54) for the transverse acoustic wave can be written as:

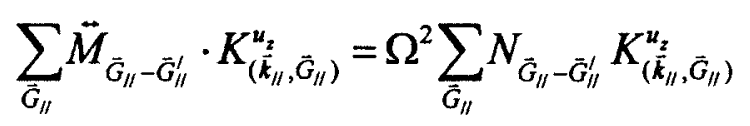




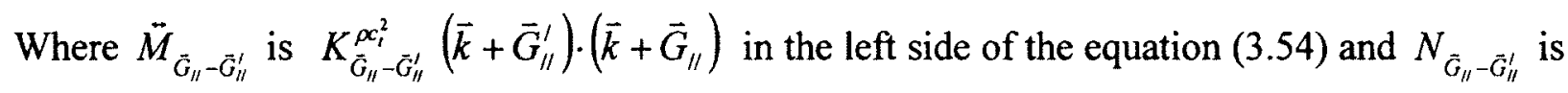
$K_{\bar{G}_{j}-\bar{G}_{n}^{\prime}}^{\rho}$ in the right hand side of the equation (3.54). This equation can be rewritten in a matrix form:

$$
\ddot{M} \cdot K_{\left(\bar{k}_{l l}, \bar{G}_{l l}\right)}^{u_{u}}=\Omega^{2} N K_{\left(\overline{\bar{k}_{l}}, \bar{G}_{l l}\right)}^{u_{z}}
$$

Therefore the matrix $\bar{A}$ defined in equation (3.55) is given by

$$
\vec{A}=N^{-1} \vec{M}
$$

This gives the form of plane wave equation (3.55) for the equation (3.54), the plane wave method can be apply to that equation easily and with less time consumtion.

\subsubsection{MATLAB Programming of Transverse Acoustical Eigenvalue Equations}

The eigenvalue equation (3.55) was implemented in MATLAB and a copy of the program is in Appendix C. Results for the transverse acoustic wave was checked against published papers ${ }^{40}$. For the square structure of material parameters $\rho_{b}=2.697 \mathrm{gm} / \mathrm{cm}^{3}, \rho_{a}=8.936 \mathrm{gm} / \mathrm{cm}^{3}$, $\left(\rho c_{t}\right)_{b}=2.79 \mathrm{gm}^{3} / \mathrm{s}^{2}$, and $\left(\rho c_{t}\right)_{a}=7.54 \mathrm{gm}^{3} / \mathrm{s}^{2}$. The band structure calculations using my program software for this phononic structure are shown in figure 3.6. The results well match the published paper results. 


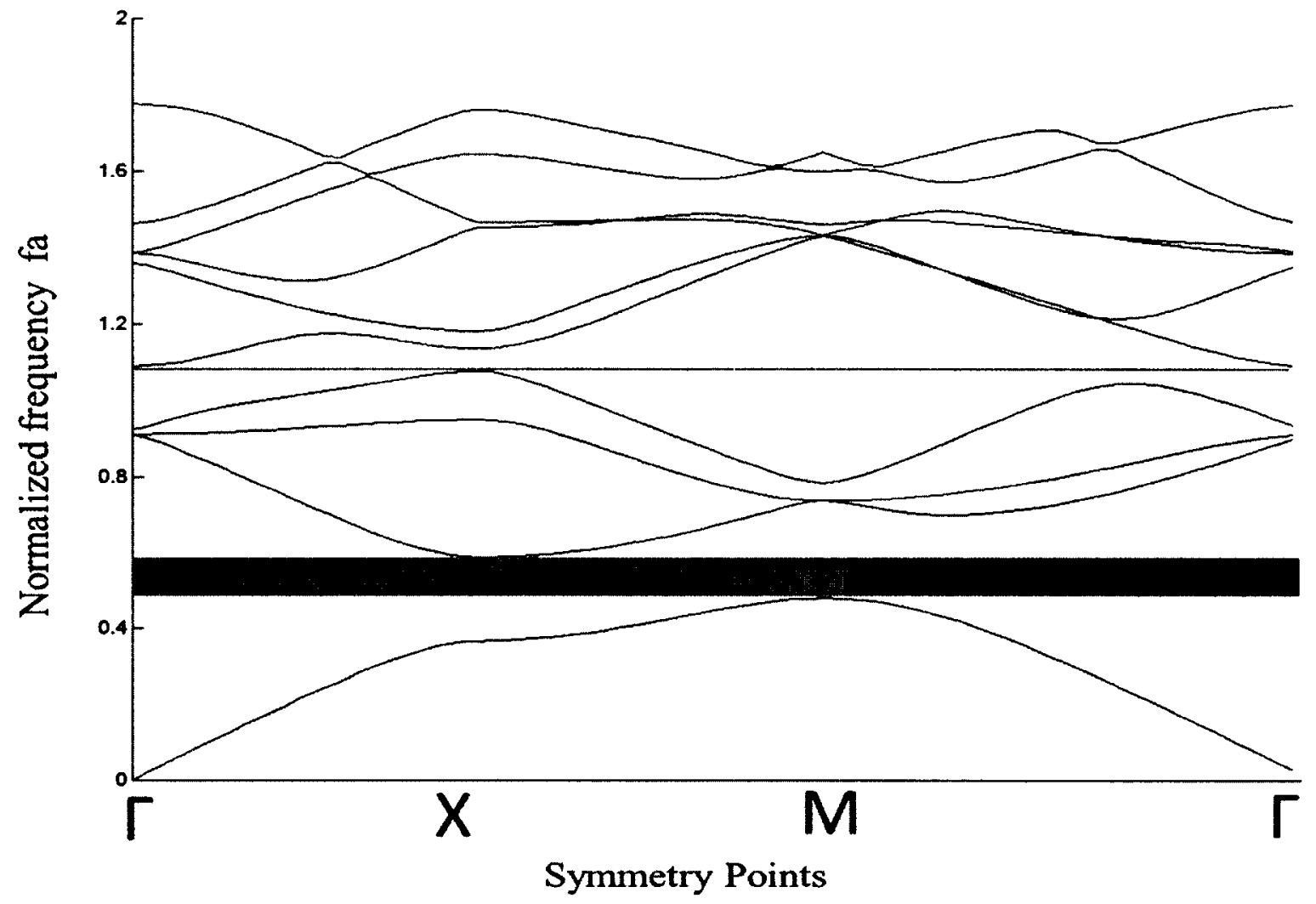

Figure 3.6: The band structure obtained using the MATLAB program of Appendix $C$ and the results published in Ref.40 .

\subsection{Acousto-Optic Interaction.}

When an acoustic wave propagates through an elastic medium, the medium's density is altered by the stresses induced by the wave ${ }^{41}$. This in turn affects the refractive index of the medium, altering its periodicity in a manner proportional to the acoustic frequency and amplitude intensity. Such acoustic-optical effects can be harnessed to create precision-tuneable photonic 
crystals $^{41}$. As the frequencies of acoustic waves are much lower than that of optical waves, the changes in refractive index induced by acoustic waves may be considered as steady relative to optical field. The change in refractive index is determined using the following equation ${ }^{41}$ :

$$
\Delta n_{i}=P_{i j} s \quad i, j=(1,2, \ldots, 6)
$$

where $P_{i j}$ is the photo-elastic coefficient tensor and $\mathrm{s}$ is the shape variation of the medium. For example, for an acoustic wave propagating in the $\mathrm{x}$ direction, the refractive index variation is given by ${ }^{41}$ :

$$
s=S \sin (w t-k z)
$$

Changing the amplitude intensity (S) of the acoustic wave changes the width of the photonic band gap, while changing of the frequency $(w)$ changes its location level. 


\subsection{Phoxonic Structure}

\subsection{Introduction}

In this chapter, the design process leading to a phoxonic crystal structure that contains a full optical band gap and transverse wave acoustical band gap is outlined. The design process consisted of first seeking out a structure that contained a full optical band gap, then determining if the same structure contained a band gap for the transverse acoustical wave. Modifications to the unit cell were performed in order to "optimize" the band gap presence. The base starting unit cell was composed of both high and low dielectric regions patterned into a square array. The starting unit cell was then modified to contain multiple and different inclusion shapes and to replace the air regions with a polymer. A composite, silicon-polymer, with multiple shape inclusions is a new approach to solid-solid phoxonic crystal structure design. As indicated in the introduction, one of the purposes of the research is to determine a square array structure with multiple inclusions in the unit cell which will support a full optical and transverse acoustical band gaps simultaneous, a phoxonic band gap. In the next chapter, waveguides are explored in the final structure of this chapter. It should be noted that the reverse engineering of a composite structure from a proposed band structure is not possible. Thus the process in band structure and band gap engineering always proceeds as a trial and adjust process starting from proposed unit cell and lattice geometry and then computing the band diagrams.

\subsection{Different Shape of Inclusions}

The first structure studied consisted of a simple pattern of square and circular cross-section silicon inclusions in an air matrix as shown in figure 4.1. In this structure the radius of the circle cross-section $r_{h}=0.2 \mathrm{a}$, and the half side length of square is $L=0.3 \mathrm{a}$. The inclusions are the 
high dielectric material (silicon $\varepsilon_{h}=12.11504$ ) and the background material is the low dielectric material air $\left(\varepsilon_{l}=1\right)$. The structure can be reconstructed using the square unit cell arranged in square array. Therefore, this structure has square lattice geometry. This structure and the unit cell are shown in figure 4.1 .

Using the MATLAB programs in Appendix B for optical, the TM and TE band diagrams of the structure shown in figure 4.1 were calculated. For accurate results 441 plane waves were used. The band gaps are shown as the grey regions in the band diagrams. As can be seen in Figure 4.2, this structure exhibits a complete optical band gap. It can be noticed that the complete band gap in this air-solid structure is not large. In addition, air-silicon composite cannot support a transverse displacement as the silicon is disconnected. Therefore, this structure needs to be modified. First the structure is converted to a solid-solid configuration that can support the transvers acoustical wave. Second, the cell structure is changed in order to find the full optical band gap widths.
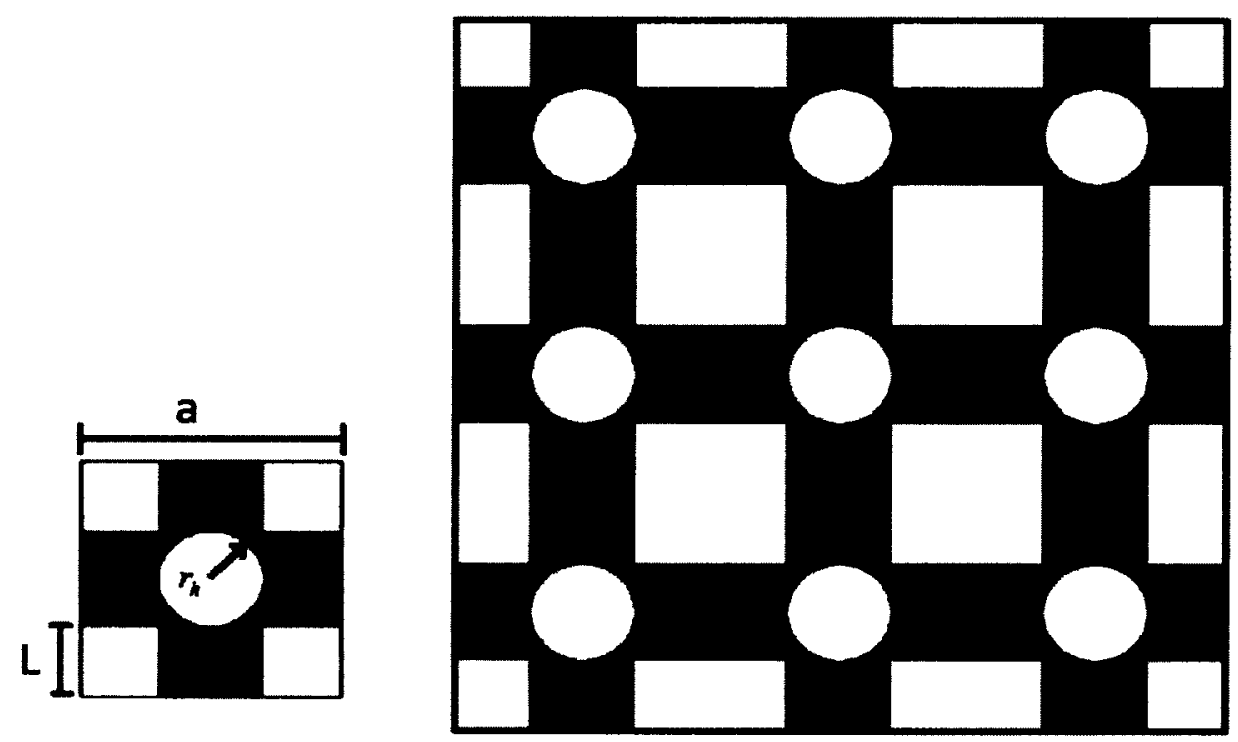

53 | Chapter 4: Phoxonic Structure 
Figure 4.1: Left: square and circle cross-section inclusions for one unit cell. Right: $3 \times 3$ array; white for high dielectric silicon $\left(\varepsilon_{h}=12.11504\right)$ and black for low dielectric air $\left(\varepsilon_{l}=1\right)$. The radius of the circle cross-section $r_{h}=0.2 \mathrm{a}$, and the half length of the square side is $L=0.3 \mathrm{a}$.

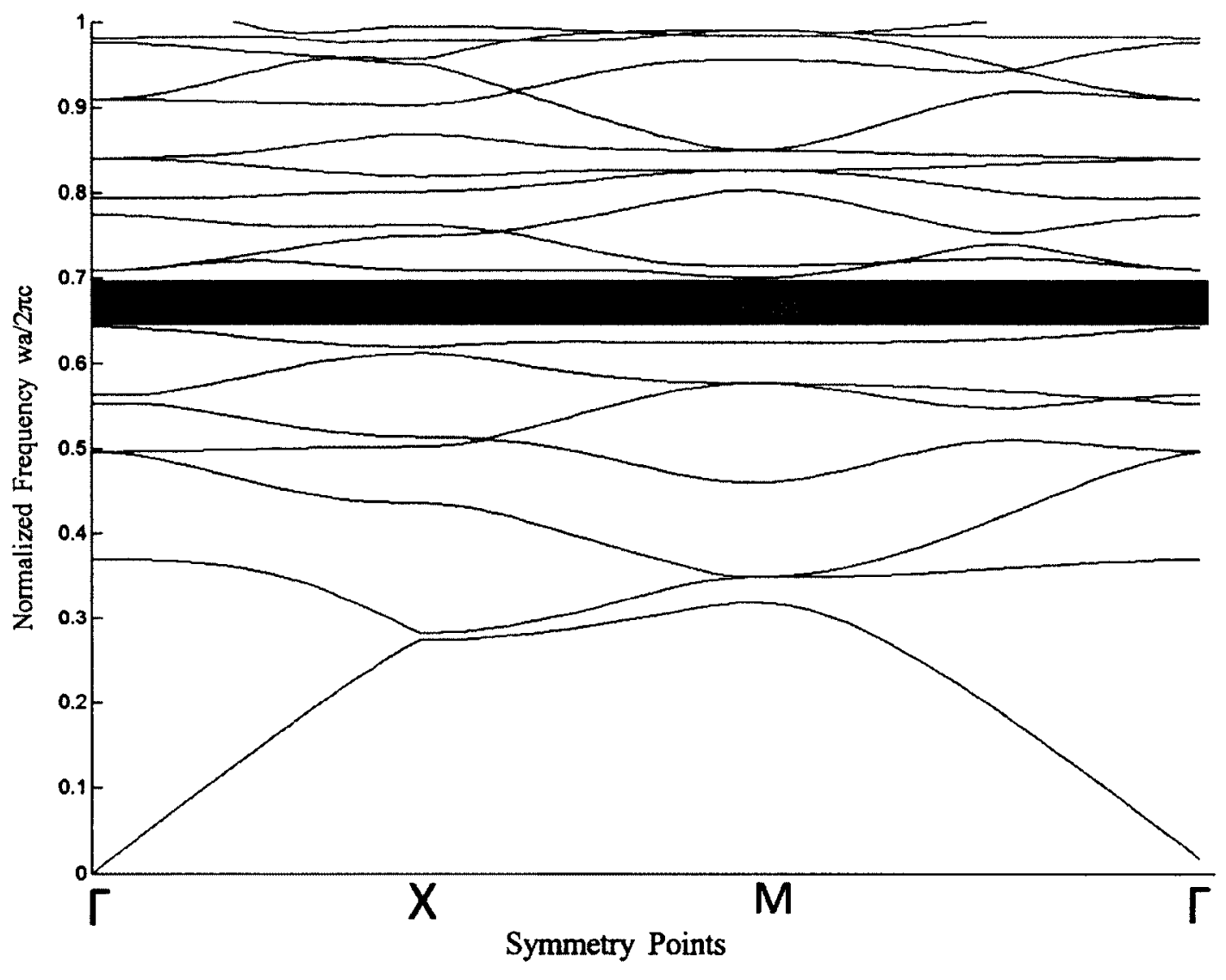




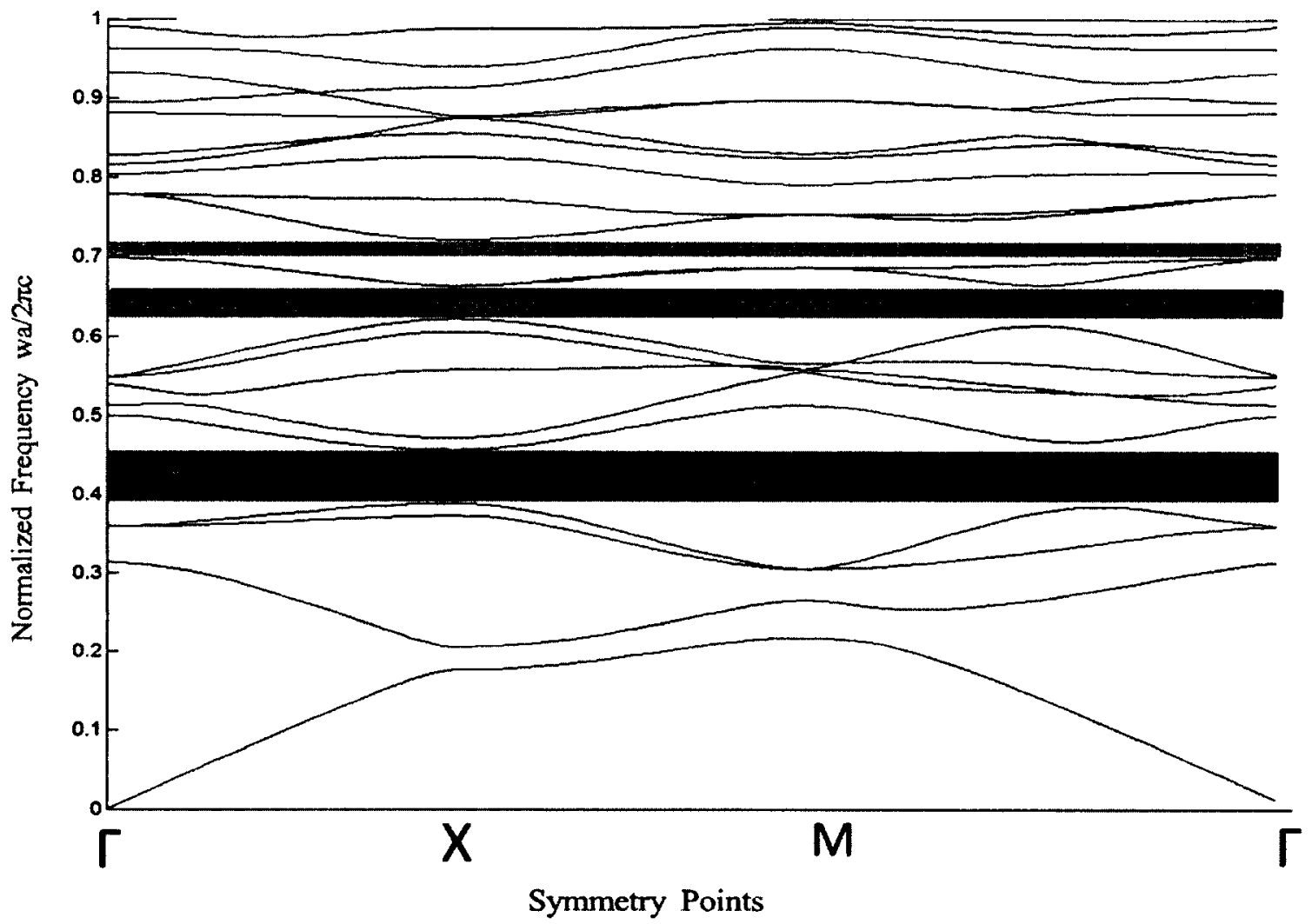

Figure 4.2: Complete optical band gap of the photonic crystal structure of figure 4.1. The radius of the circle cross-section $r_{h}=0.2 \mathrm{a}$, and the half length of the square side is $L=0.3 \mathrm{a}$. For high dielectric silicon $\left(\varepsilon_{h}=12.11504\right)$ and black for low dielectric air $\left(\varepsilon_{l}=1\right)$. Top: TE. Bottom: TM.

\subsection{Solid-Solid Structure (Silicon and polymer)}

Photonic band gaps are not commonly exhibited by solid-solid structures due to their low refractive index contrast. As we have seen, band gaps can be present in air-solid structures with square lattices by using different inclusion shapes. However, in a solid-solid structure complete band gaps tend to close. The same structure parameters as in figure 4.1 have used but the air is 
replaced by polymer, the complete band gap closed (see figure 4.3). To compensate the geometry of the lattice is modified in an attempt to obtain the desired optical band gap.

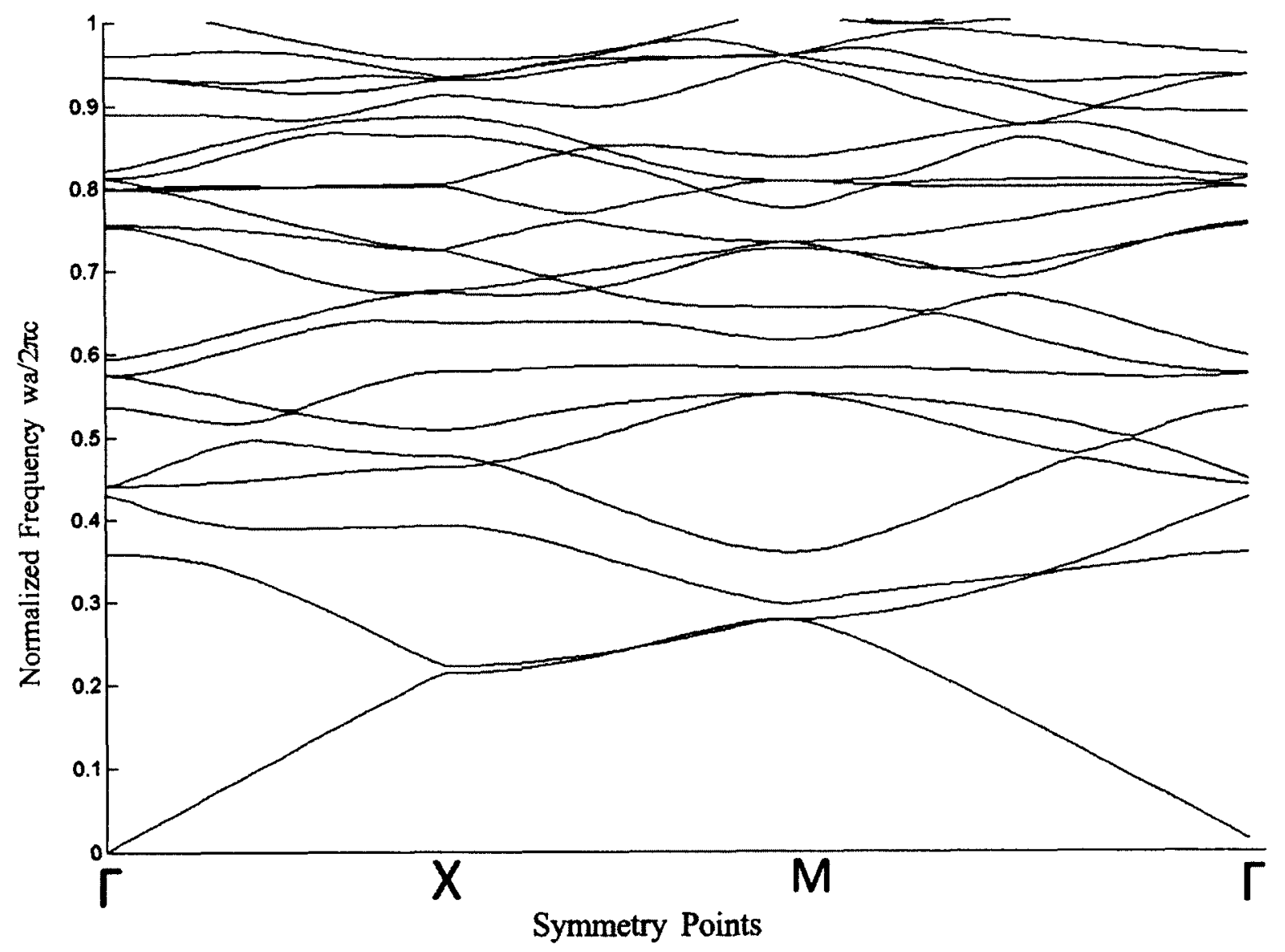

56/Chapter 4: Phoxonic Structure 


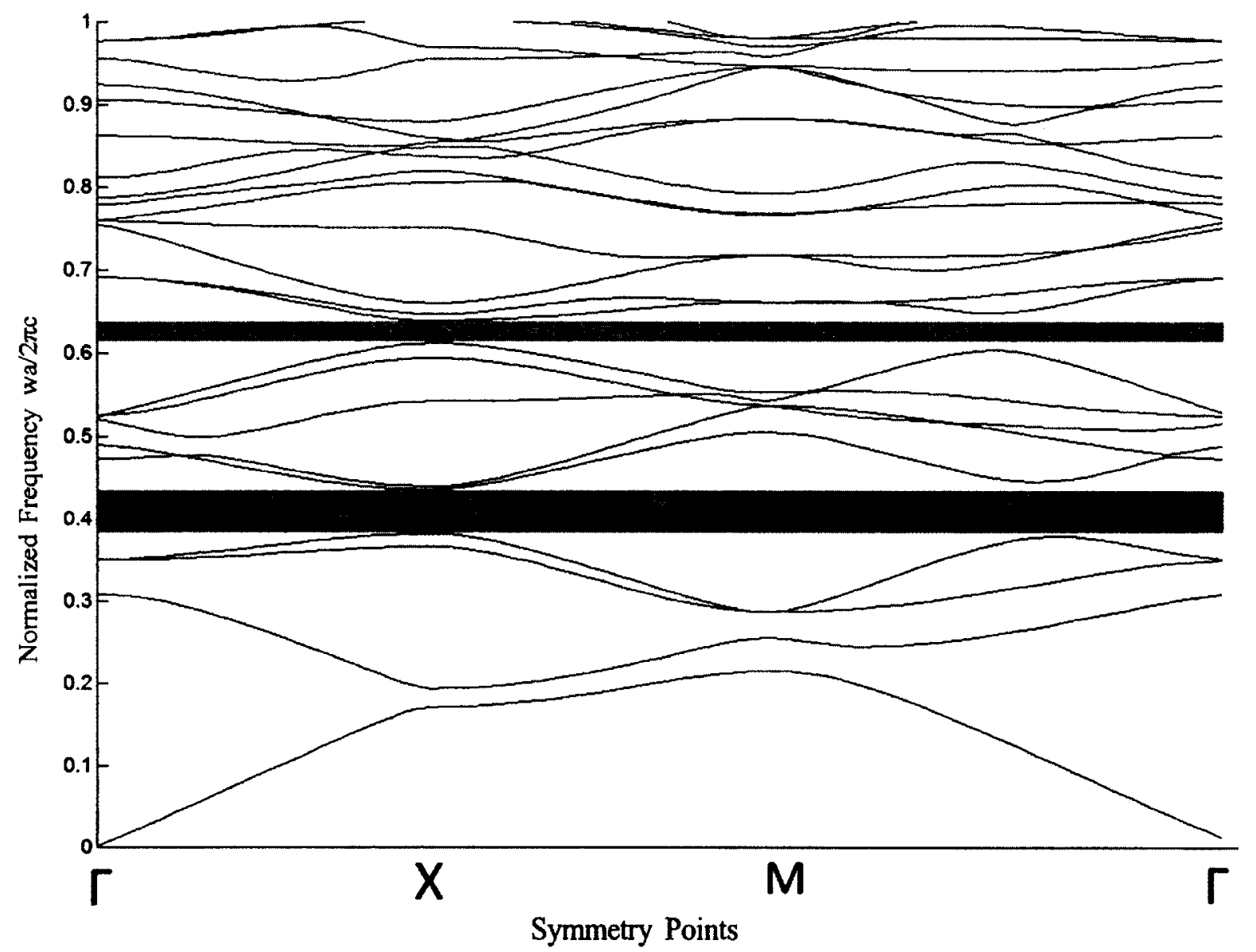

Figure 4.3: Optical band diagrams for the structure of figure 4.1. The radius of the circle crosssection $r_{h}=0.2 \mathrm{a}$, and the half length of the square side is $L=0.3 \mathrm{a}$. For high dielectric silicon ( $\left.\varepsilon_{h}=12.11504\right)$ and black for low dielectric air $\left(\varepsilon_{l}=2.4\right)$. Top: TE. Bottom: TM. Grey: band gap regions. No complete optical band gap.

A modified structure is shown in figure 4.4, left. The lattice has been rotated and the centre holes have been resized. In this structure the radius of the centre circle is $r_{h}=0.25 \mathrm{a}$, and the half length of square diagonal is $L=0.37 \mathrm{a}$. The white regions are the high dielectric material silicon $\left(\varepsilon_{h}=\right.$ 12.11504) where the black material is the low dielectric polymer $\left(\varepsilon_{l}=2.4\right)$. The $3 \times 3$ array is 
shown in figure 4.4, right. Using the MATLAB program in Appendix B the TM and TE band structures of the structure shown in figure 4.5 were calculated. For accurate calculations 441 plane waves were used. In figure 4.5 grey indicates the band gap regions and the black band in TM diagram indicate the overlap region.
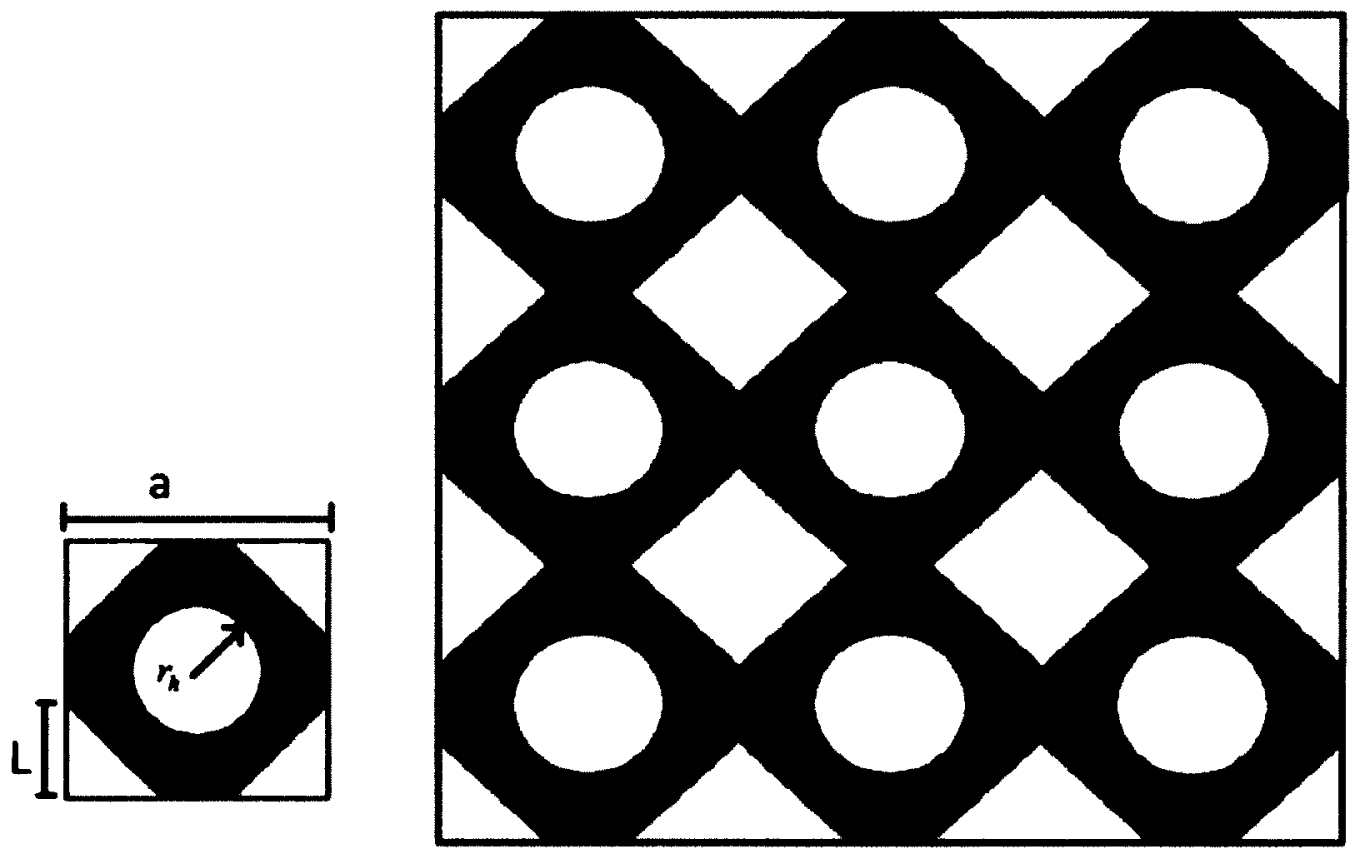

Figure 4.4: Left: rotated square and circle cross-section inclusions for one unit cell. Right: $3 \times 3$ array; white for high dielectric (silicon $\varepsilon_{h}=12.11504$ ), and black for low dielectric (polymer $\varepsilon_{l}=2.4$ ). Radius of the centre circle is $r_{h}=0.25 \mathrm{a}$, and the half length of square diagonal is $L$ $=0.37 \mathrm{a}$. 

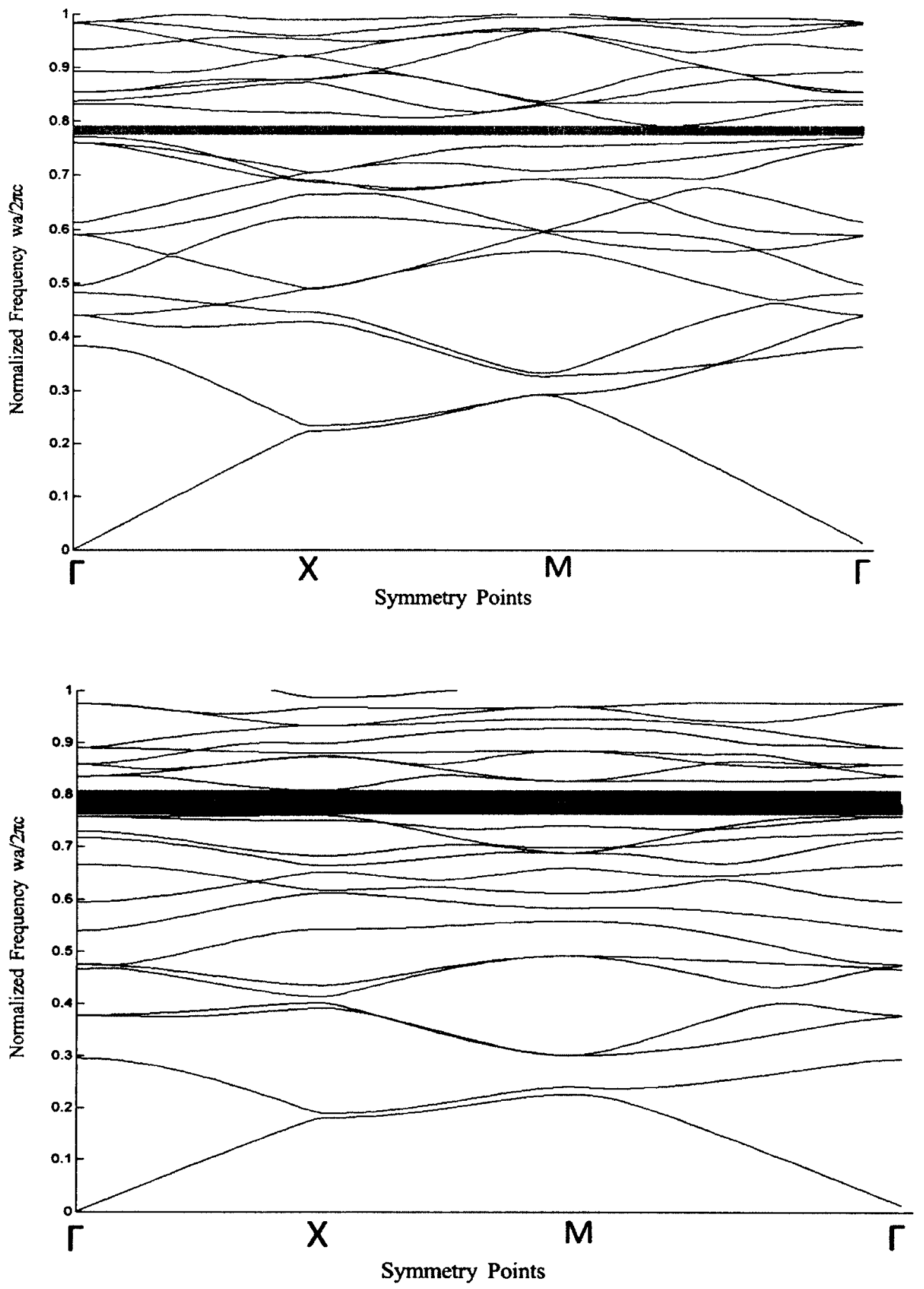

Figure 4.5: Optical band diagrams for the structure of figure 4.4. Radius of the centre circle is $r_{h}$ $=0.25 \mathrm{a}$, and the half-length of square diagonal is $L=0.37 \mathrm{a}$. For high dielectric silicon $\left(\varepsilon_{h}=\right.$ 59 /Chapter 4: Phoxonic Structure 
12.11504) and black for low dielectric air $\left(\varepsilon_{l}=2.4\right)$. Top: TE. Bottom: TM. Grey: band gap regions. Black: overlap band gap regions.

The complete band gap is small, but the modifications to the cell have resulted in a full band gap that is large with the polymer present than the air-solid full band gap of figure 4.2. Previous research indicates that solid-inclusion/gas matrix structures are superior for opening TM band gaps while gas inclusion/solid matrix structures are superior for TE band gap. In an attempt to further increase the size of the full band gap a circular hole in the rotated square was added.

The new unit cell structure is a hybrid of the two basic structures; however, further structure modification may be required as the TE and TM are not additive, as each sub-structure influences the other's band gap.

The modified structure is shown in Figure 4.6, left for a single cell and right for $3 \times 3$ array, featuring two different inclusion shapes: a hollow circular cross-section and a hollow square cross-section with an embedded solid circle.
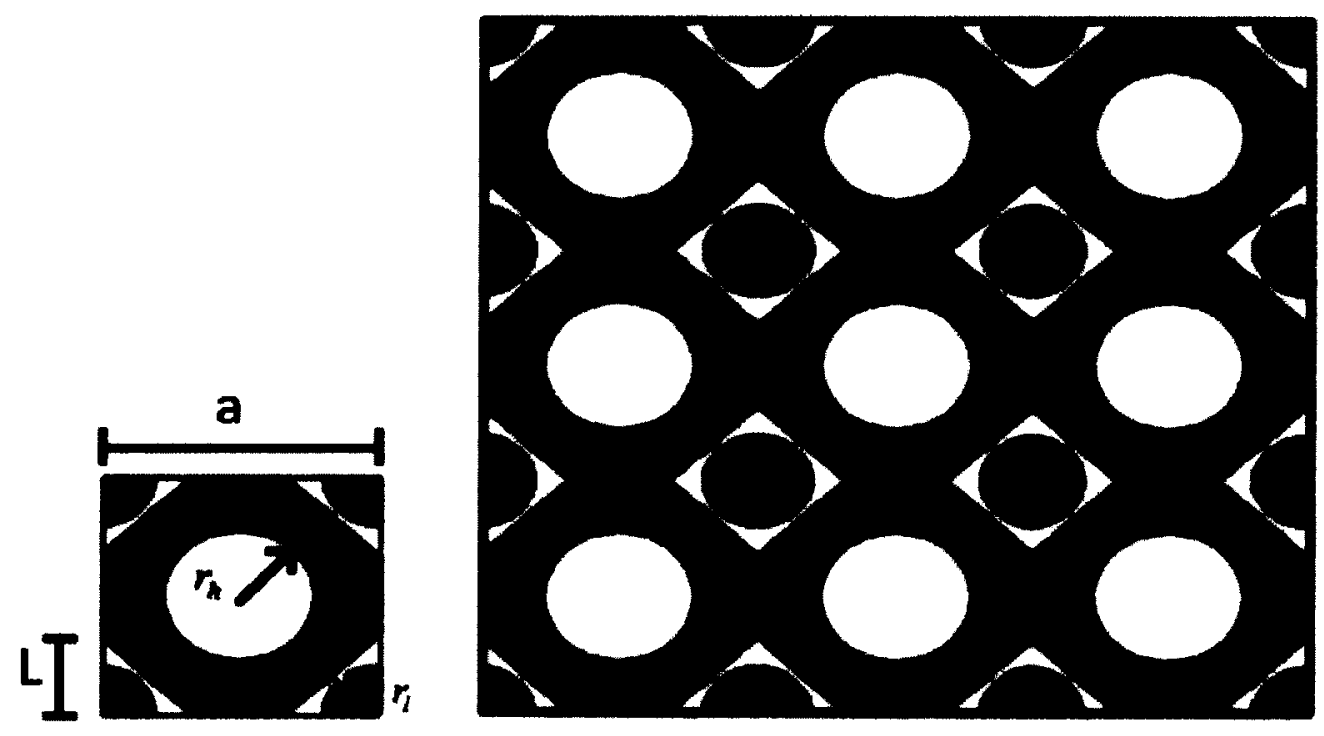

60|Chapter 4: Phoxonic Structure 
Figure 4.6: Modified hybrid crystal structure. The white regions are high-dielectric silicon $\left(\varepsilon_{h}=\right.$ 12.11504 , mass density $2.33 \mathrm{~g} / \mathrm{cm}^{3}$, speed of sound $5360 \mathrm{~m} / \mathrm{s}$ ), the black regions are lowdielectric epoxy of polymer $\left(\varepsilon_{l}=2.4\right.$, mass density $1.1 \mathrm{~g} / \mathrm{cm}^{3}$, speed of sound $\left.2650 \mathrm{~m} / \mathrm{s}\right)$. Left: one unit cell. Right: $3 \times 3$ arrays. Radius of the centre circle is $r_{h}=0.26 \mathrm{a}$, radius of the corner circle $r_{l}=0.2$, and the half-length of square diagonal is $L=0.32 \mathrm{a}$.

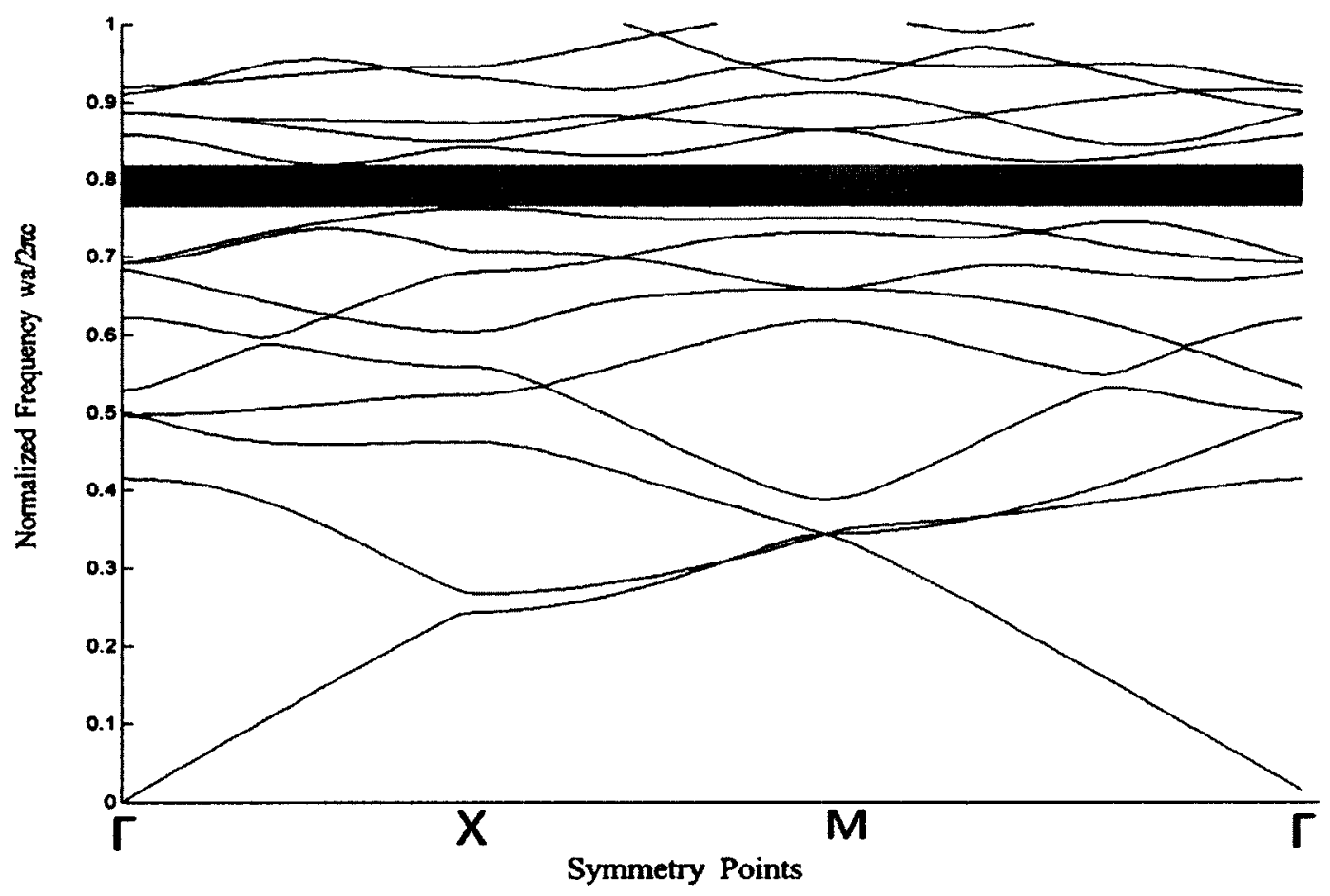

61 IChapter 4: Phoxonic Structure 


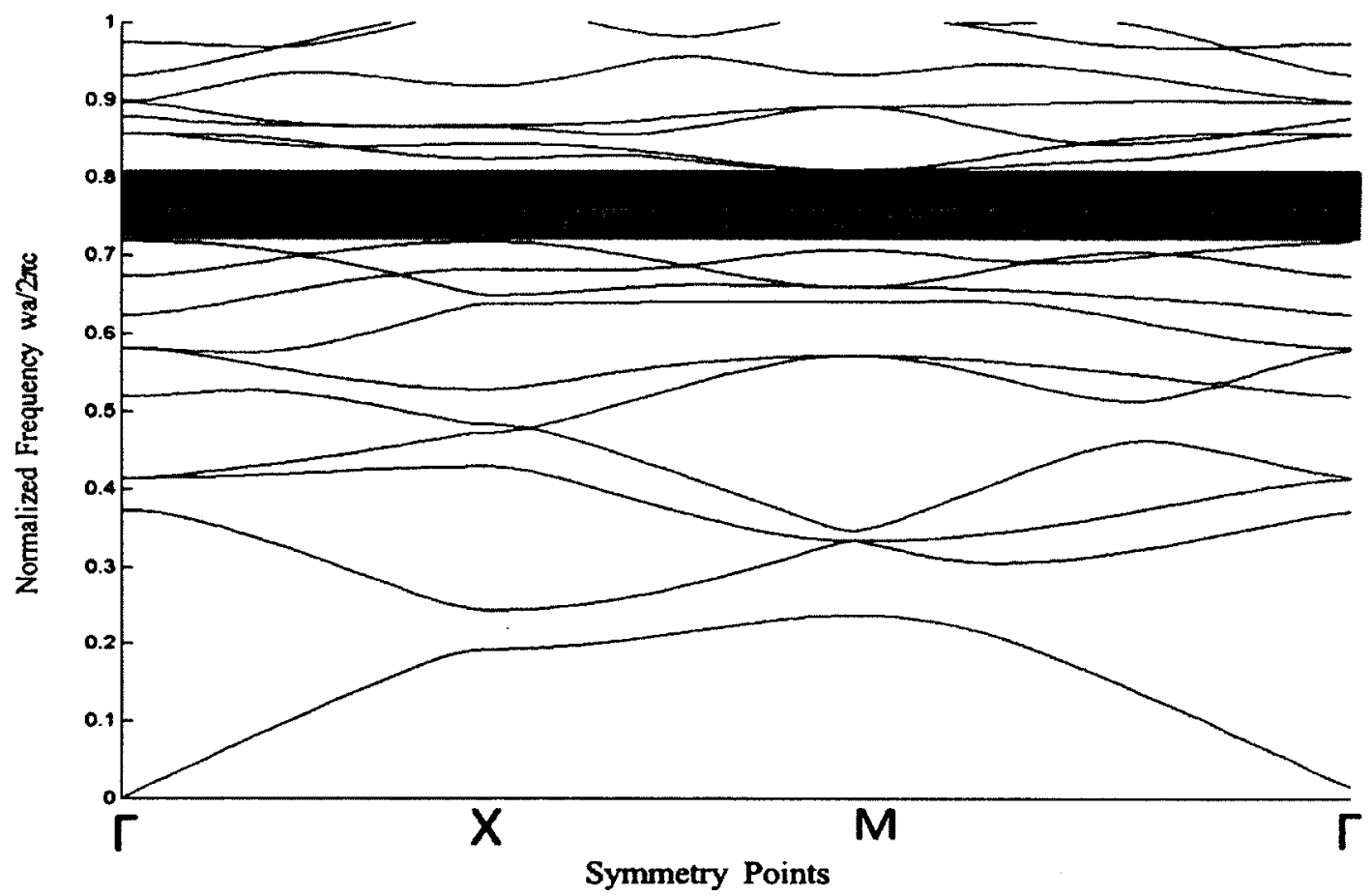

Figure 4.7: Band gap for the structure shown in figure 4.6. Radius of the centre circle is $r_{h}=$ $0.26 \mathrm{a}$, radius of the corner circle $r_{1}=0.2$, and the half-length of square diagonal is $L=0.32 \mathrm{a}$. Top: TE. Bottom: TM. Grey: band gap regions. Black: overlap band gap regions.

Three parameters may be modified $r_{h}, r_{l}, \mathrm{~L}$, and the full band gap determined for the parameters combination the structure showing the largest full band gap has used. In this structure the radius of the circle cross-section $r_{h}=0.26 \mathrm{a}$, the half-length of square diagonal is $L=0.32 \mathrm{a}$, and the circle cross-section of low dielectric embedded inclusion $r_{l}=0.2 \mathrm{a}$. The inclusions are the high dielectric silicon $\left(\varepsilon_{h}=12.11504\right)$, where the background material is the low dielectric polymer, ( $\left.\varepsilon_{l}=2.4\right)$. Figure 4.7 shows the band structures, band gaps and complete optical band gaps for TE and TM. 
The band gap width is calculated using the ratio of the band width over the centre band frequency $\Delta w / w_{c}$. The complete band gap width of the band diagram shown in figure 4.6 is about $7 \%$ of the frequency in the band gap centre. In the next section, the acoustical band diagram will be examined for the best parameters that used for optical band diagrams.

\subsection{The Square Lattice Phoxonic Structure}

The crystal structures described in previous sections exhibited band gaps for both polarizations of light. In order to have acoustic localization and enhance interaction between optical and acoustic waves, an acoustic band gap is also necessary. From equations (3.43) and (3.48), similarity of the TE mode optical equation and transverse acoustic equation can be noted. A band gap is thus expected for transverse acoustic waves. However, the equations are not identical, therefore, a structure with an optical band gap will not necessarily feature an acoustic band gap. In the figure 4.7, the optical band structure is shown. The best parameters of the inclusions size of the previous section are used, and the band structure for acoustic waves is shown in figure 4.8. 


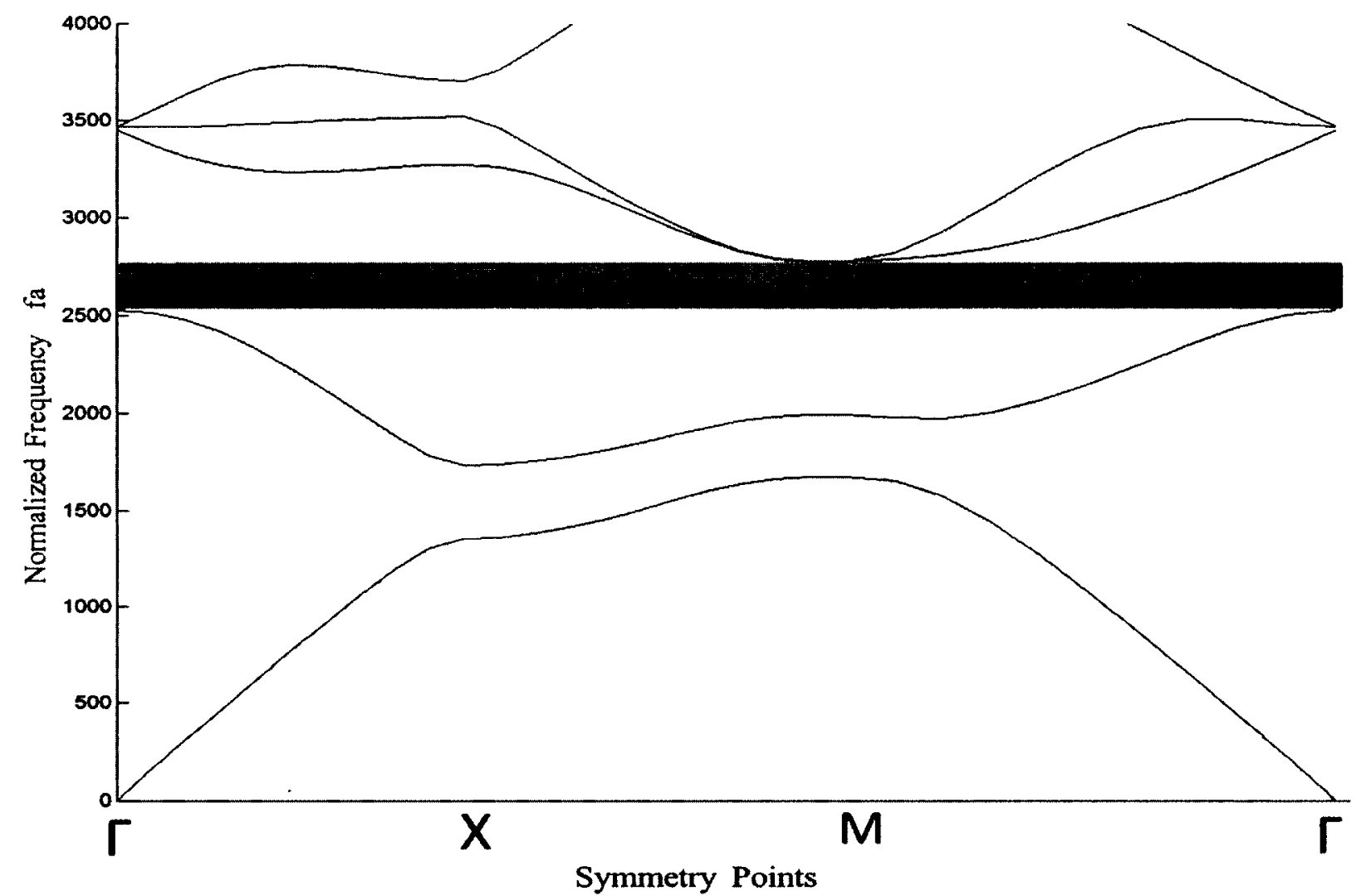

Figure 4.8: Transverse acoustic band gap for the structure shown in figure 4.6. Radius of the centre circle is $r_{h}=0.26 \mathrm{a}$, radius of the corner circle $r_{t}=0.2 \mathrm{a}$, and the half-length of square diagonal is $L=0.32 \mathrm{a}$.

For photonic crystals the band structure is represented as a function of the normalized frequency $w a / 2 \pi c$, for phononic crystals it is expressed as a function of normalized frequency $f a$, where $f$ is the acoustic frequency and (a) is the period of the crystal. By using two different shapes of inclusions in the structure, a complete optic band gap of $7 \%$ is presented. An acoustic band gap with a wide frequency range is also presented of $9 \%$ bandwidth of the frequency in the band gap centre. The complete optical band gap is found for normalized electromagnetic frequencies 
$w a / 2 \pi c$ between 0.75 and 0.81 , with the mid-gap frequency at 0.78 . For the telecommunication wavelength of $1550 \mathrm{~nm}$ and recognizing that normalized frequency $w a / 2 \pi c$ which may also be written in terms of the free space wavelength as $a / \lambda$, the lattice constant should be $1.2245 \mu \mathrm{m}$. The lattice constant of $1.2245 \mu \mathrm{m}$ yields a minimum separation distance between different dielectric elements of $37.73 \mathrm{~nm}$, well within the capabilities of nano-scale fabrication techniques (see figure 4.9). The phononic band gaps lie between 1700 to 1750 and 2550 to 2750 , respectively. The corresponding acoustic mid-gap lies in the low- normalized frequency range around 2650, making fabrication using available technology feasible. The acoustic frequency can be calculated by dividing the mid-gap normalized frequency value (about 2650) to the lattice constant $(1.2245 \mu \mathrm{m})$ and that will be $2.16 \mathrm{GHz}$. Therefore the acoustic wavelength is the speed of sound divided by the acoustic frequency $\lambda=c_{t} / f$, this will result as $\lambda=1.2 \mu \mathrm{m}$ for the polymer material .

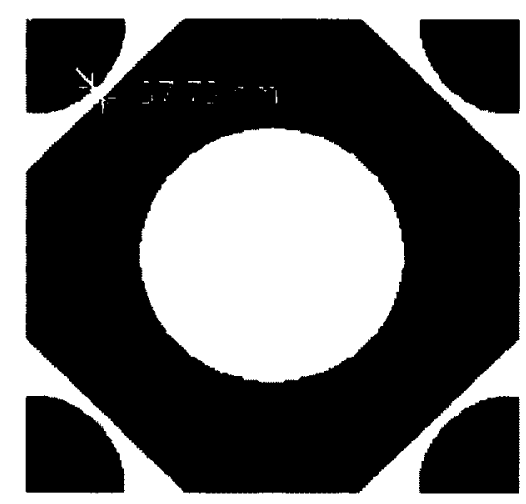

Figure 4.9: The lowest speration in the final unit cell. For nano scale fabrication this minimum is large enougth.

\subsection{Bandgap Maps for Optical and Acoustical Domain}

65/Chapter 4: Phoxonic Structure 
To explore the sensitivity of the full band gap to cell parameters variations band gap maps were made. In these maps the band gaps are calculated for each one of the parameter variation, such as the radius of the circle cross section of the high dielectric inclusion and the lattice constant, the radius of the circle cross section of the low dielectric inclusion and the lattice constant, or the half length of the diagonal of the square cross section inclusion and the lattice constant. These maps are shown for optical in figure 4.10,4.11 and 4.12, respectively. While for acoustic waves the gap maps are shown in figure $4.13,4.14$, and 4.15 , respectively.

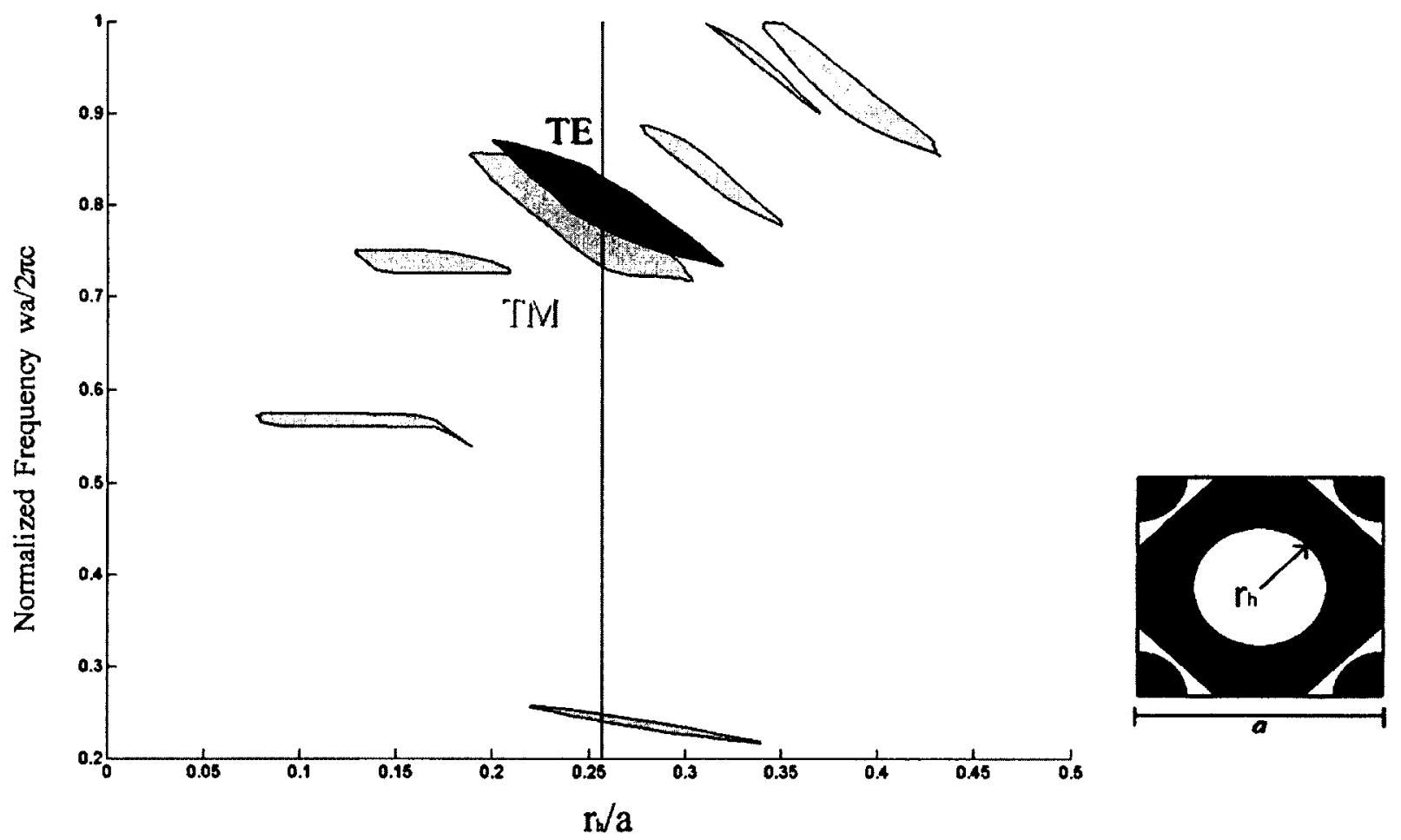

Figure 4.10: Optical band gap map for the structure shown in Figure 4.6, plotted as a function of the centre circle radius and the lattice constant. The half length of square diagonal is 
fixed at $L=0.32 \mathrm{a}$, and the corner circle is fixed at $r_{l}=0.2$. The vertical line is the design value for $r_{h} / a$.
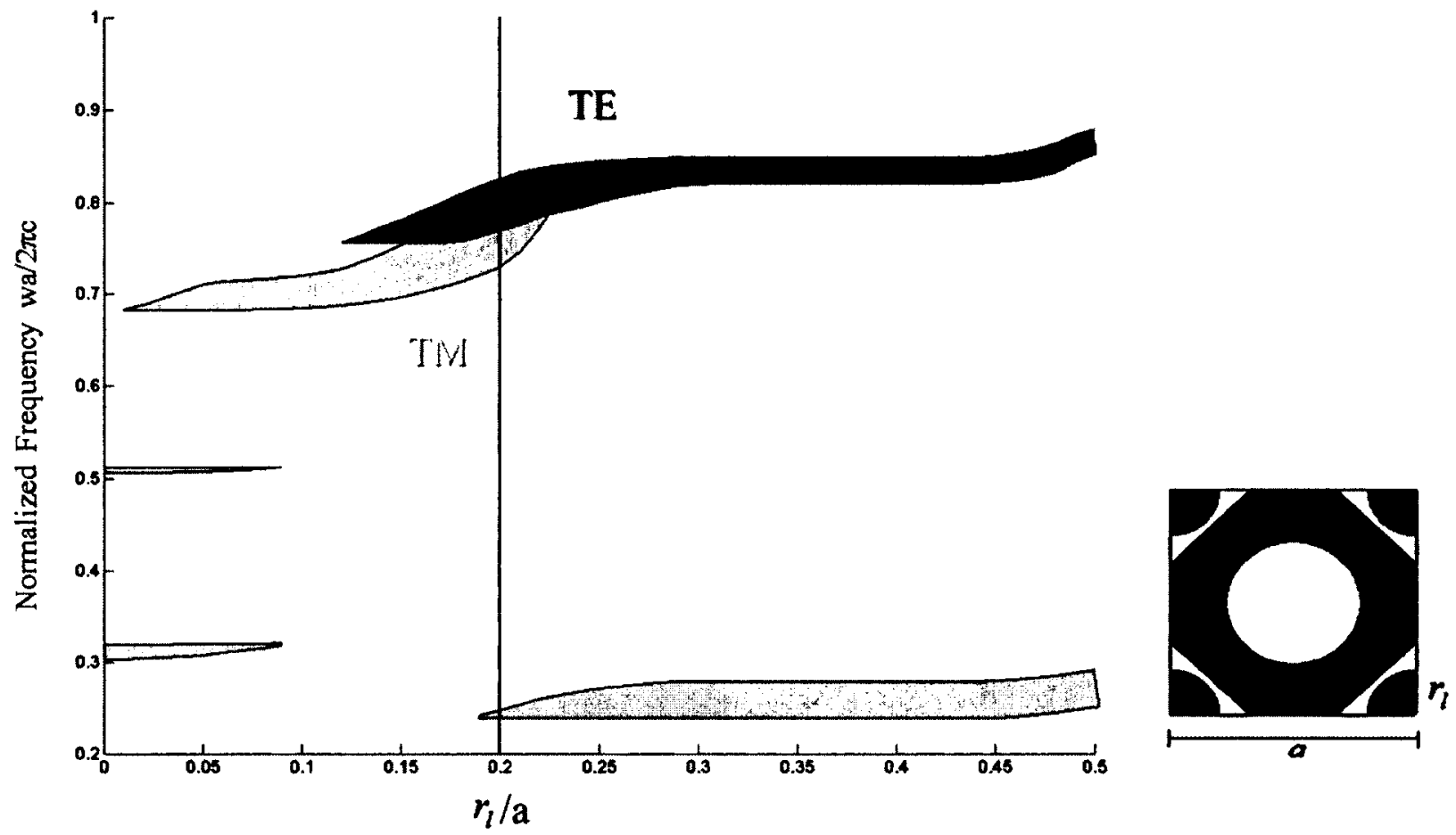

Figure 4.11: Optical band gap map for the structure shown in Figure 4.5, plotted as a function of the corner circle radius and the lattice constant. The half length of square diagonal is fixed at $L$ $=0.32 \mathrm{a}$, and the centre circle is fixed at $r_{h}=0.26$. The vertical line is the design value for $r_{l} / a$. 


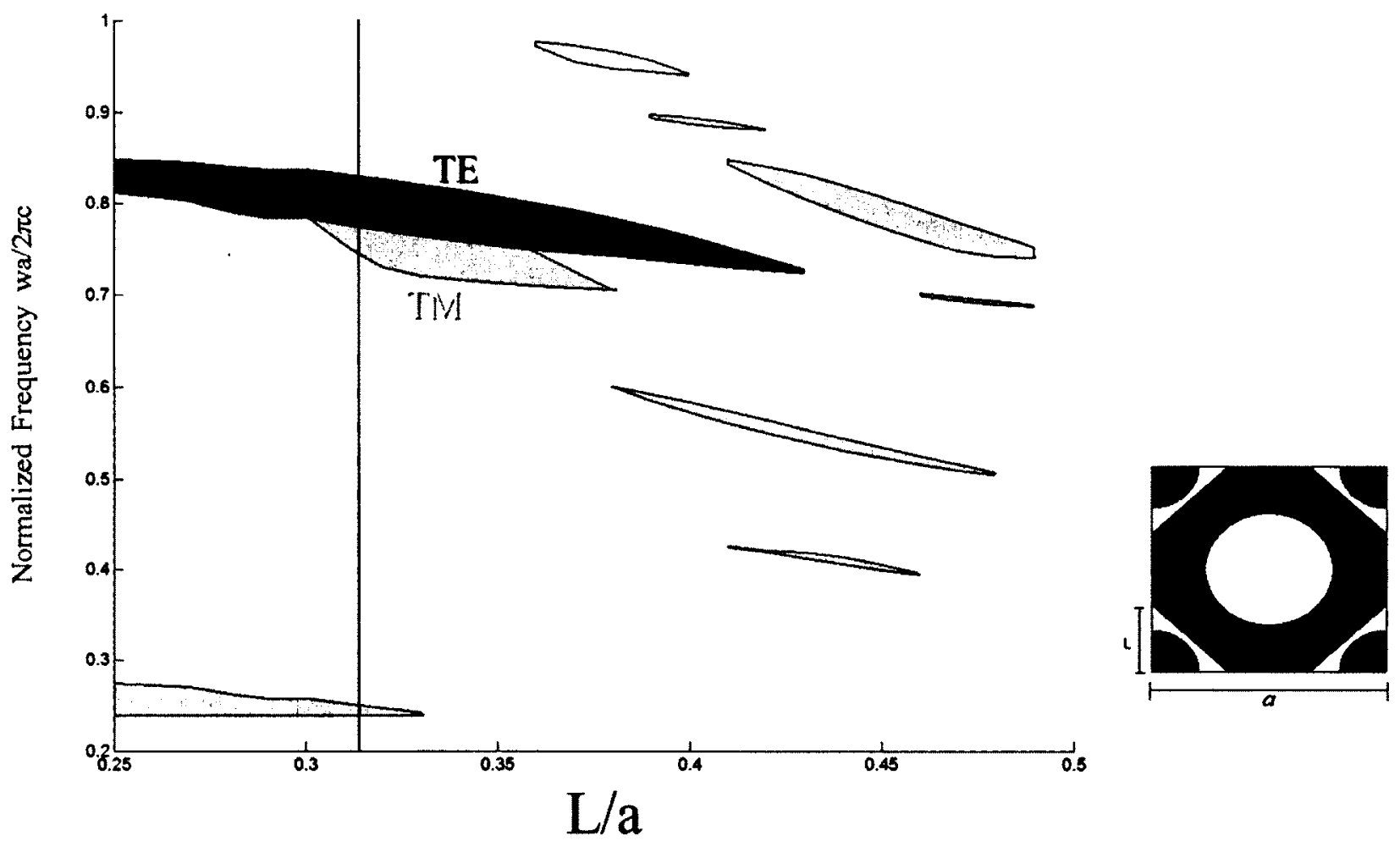

Figure 4.12: Optical band gap map for the structure shown in Figure 4.5, plotted as a function of the diagonal square size half length and the lattice constant $\mathrm{L} / \mathrm{a}$. The centre circle is fixed at $r_{h}=$ $0.26 \mathrm{a}$ and the corner circle is fixed at $r_{l}=0.2 \mathrm{a}$. The vertical line is the design value for $\mathrm{L} / \mathrm{a}$.

Figures 4.10., 4.11 and 4.12 show non-dimensional photonic map gaps reduced frequency $(w a / 2 \pi c)$ as a function of radius of the middle inclusion rod $r_{h}$ and the lattice constant, radius of the corner circle $r_{l}$ and the lattice constant, and length of half-length of square diagonal (L) and the lattice constant, respectively (where $\mathrm{c}$ is the speed of light). While for acoustic, figures $4.13,4.14$, and 4.15 show the acoustic gap maps. From the band gap maps, it 
can be seen that the optimal parameters are $r_{h}=0.26 \mathrm{a}, \mathrm{L}=0.32 \mathrm{a}$ and $r_{l}=0.2 \mathrm{a}$, As indicated by the vertical lines. The full band gap is relatively stable to small parameters variations.

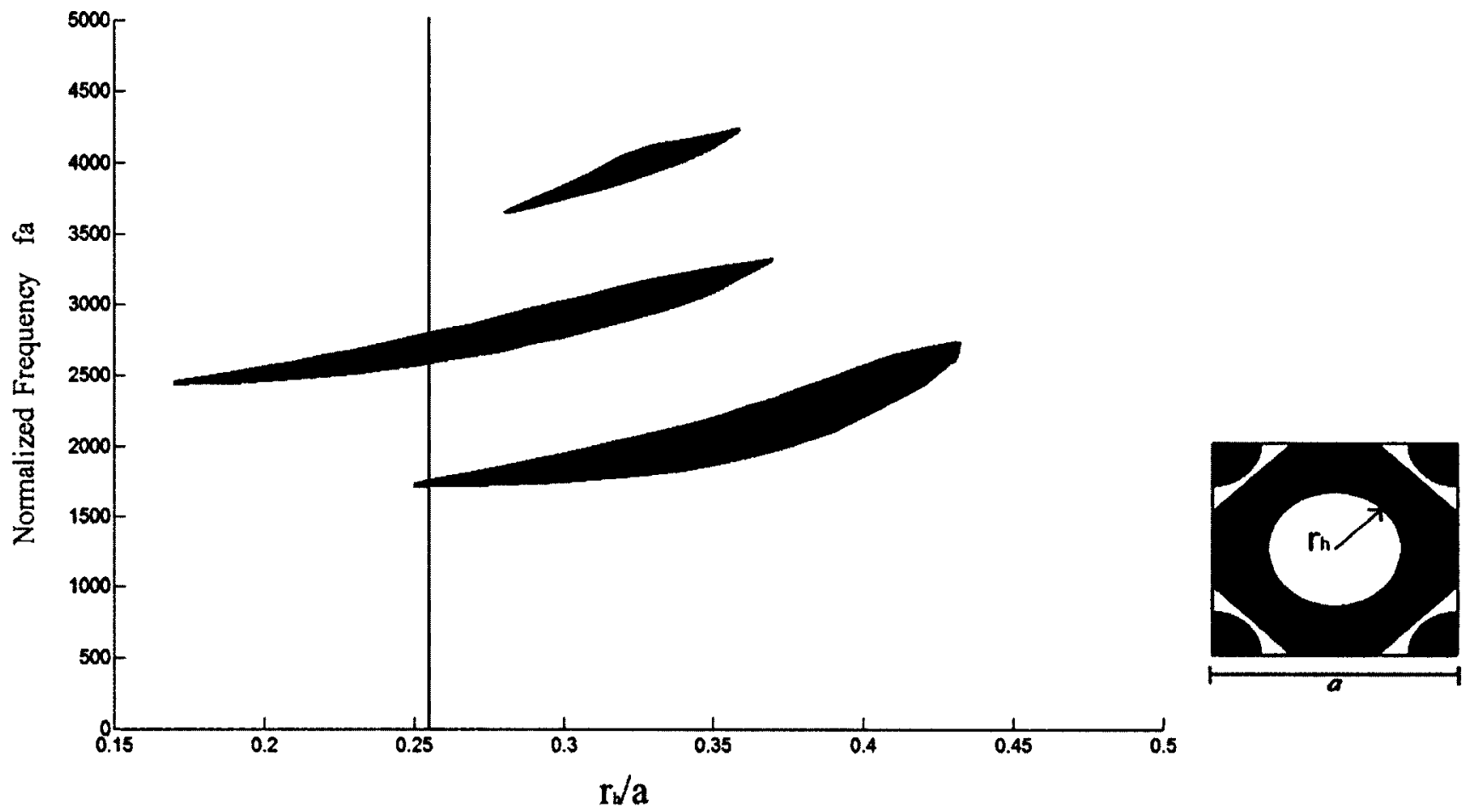

Figure 4.13: acoustics band gap map for the structure shown in Figure 4.6, plotted as a function of the centre circle radius and the lattice constant. The half length of square diagonal is fixed at $L=0.32 \mathrm{a}$, and the corner circle is fixed at $r_{l}=0.2$. The vertical line is the design value for $r_{h} / a$. 


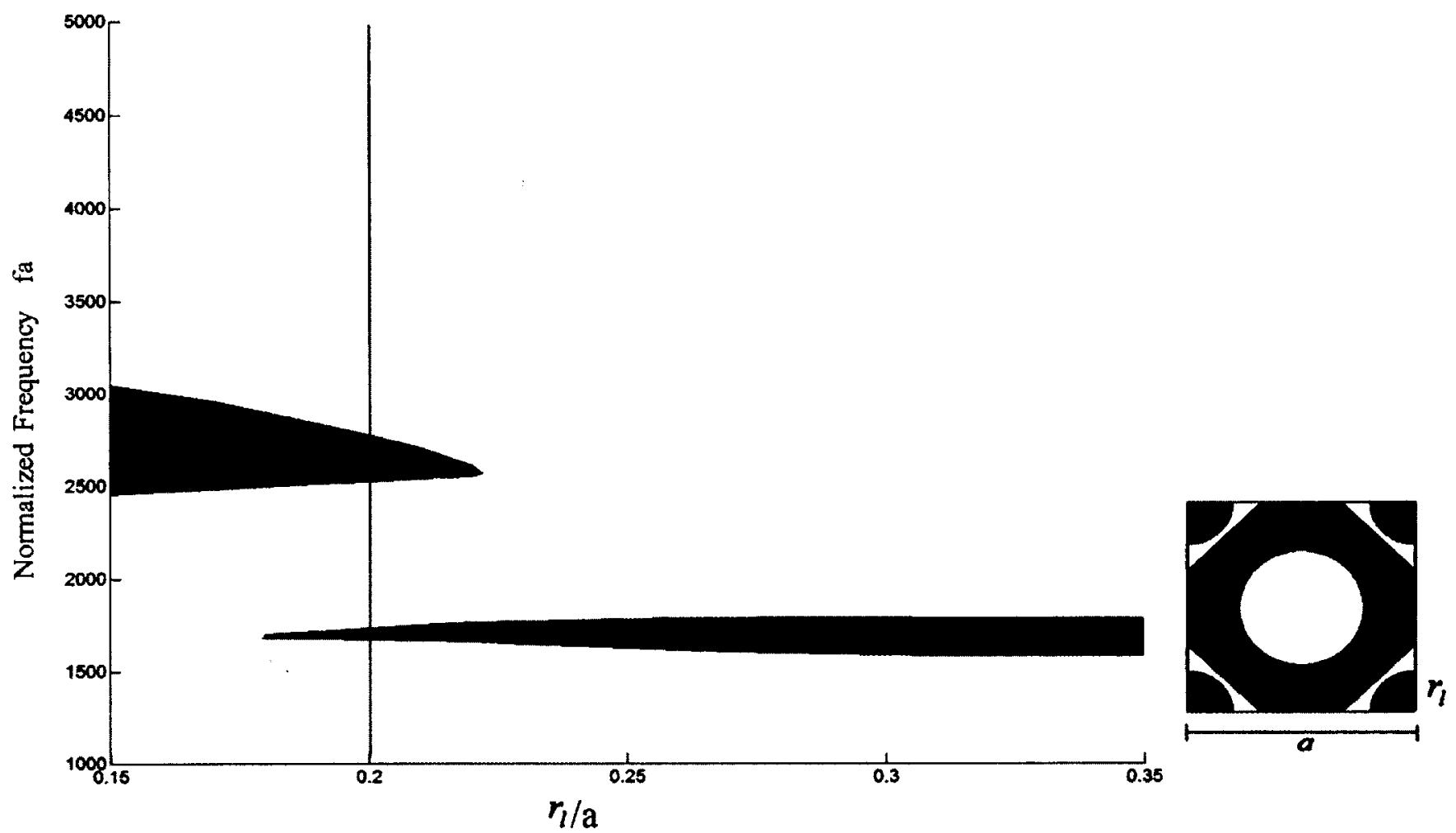

Figure 4.14: Acoustic band gap map for the structure shown in Figure 4.6, plotted as a function of the corner circle radius and the lattice constant. The half length of square diagonal is fixed at $L=0.32 \mathrm{a}$, and the centre circle is fixed at $r_{h}=0.26$. The vertical line is the design value for $r_{i} / a$. 


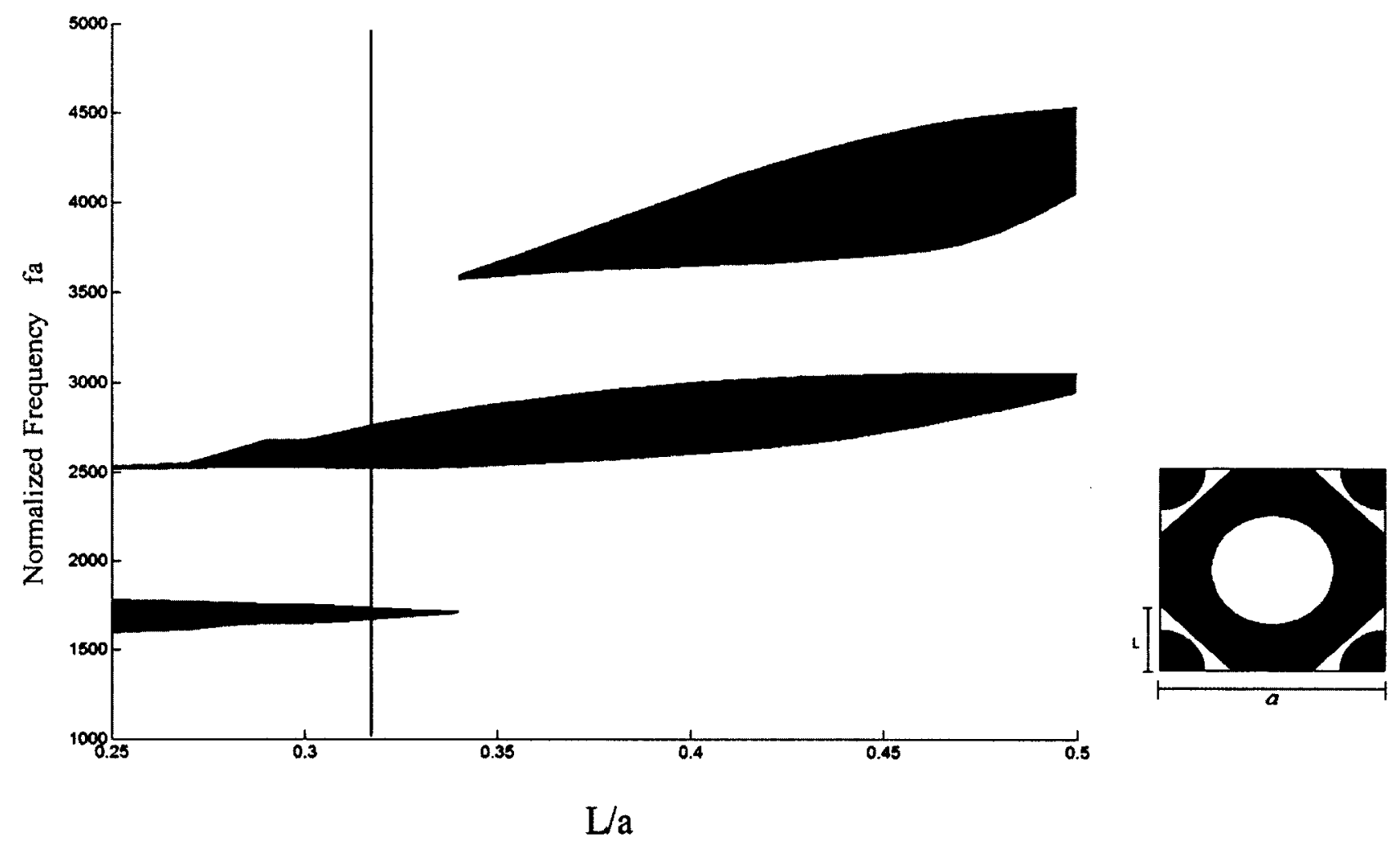

Figure 4.15: acoustic band gap map for the structure shown in Figure 4.6, plotted as a function of the diagonal square size half length and the lattice constant $\mathrm{L} / \mathrm{a}$. The centre circle is fixed at $r_{h}=$ $0.26 \mathrm{a}$ and the corner circle is fixed at $r_{l}=0.2 \mathrm{a}$. The vertical line is the design value for $\mathrm{L} / \mathrm{a}$.

As shown in gap map figures, for some of the best parameters, the small change would not affect highly the band gap width which makes this design less sensitive to the fabrication errors. However, actual structure fabrication will have some disorder and positional error. To study these imperfections, larger cells that have disorder fabrication error would be used. Of course, this process would take very long time for as numerous calculations would be required to obtain sufficiently converged results. Typically a $10 \%$ level of disorder can be tolerated before the band gap severely closes.

For solid-solid materials structure, a suitable geometry structure and the best parameters for that structure is determined In this chapter. Which exhibit full optical and transverse acoustic band 
gaps. In the next chapter, the waveguides for single mode for full optical and transverse acoustic waves will be examined. 


\section{Simultaneous Waveguide for Optical and Acoustic Waves}

\subsection{Introduction}

In the previous chapter a full optical and transverse acoustic band gaps exist for the same siliconpolymer square array structure. In this chapter, a line defect is introduced such that band gap based waveguides for optical and acoustic fields are produced.

\subsection{Supercell Method}

The plane wave method for band structure calculations requires that the unit cell will be repeated over all space. Therefore, to study the effect of a defect point or line in the photonic crystal structure using plane wave method, a supercell method is required. Using the supercell method, a line defect will be built into the structure to simultaneously localize the optical and acoustic modes. A supercell is a cell built of many original cells repeated in one or more directions and may have a defect in the centre of the repeated cell. Two such supercells are shown in figure 5.1 (left and middle), the artificial structure thus consists of periodic defects separated by regions of perfect photonic crystal (see figure 5.1 right). Of course, the unit supercell should have large dimensions to ensure good defect state calculations and mode localization. The expected band structure contains band gaps similar in width and level to the band gaps of the original photonic crystal structure, with one or more localized defect states (depending on the size of the defect) inside the band gaps. 

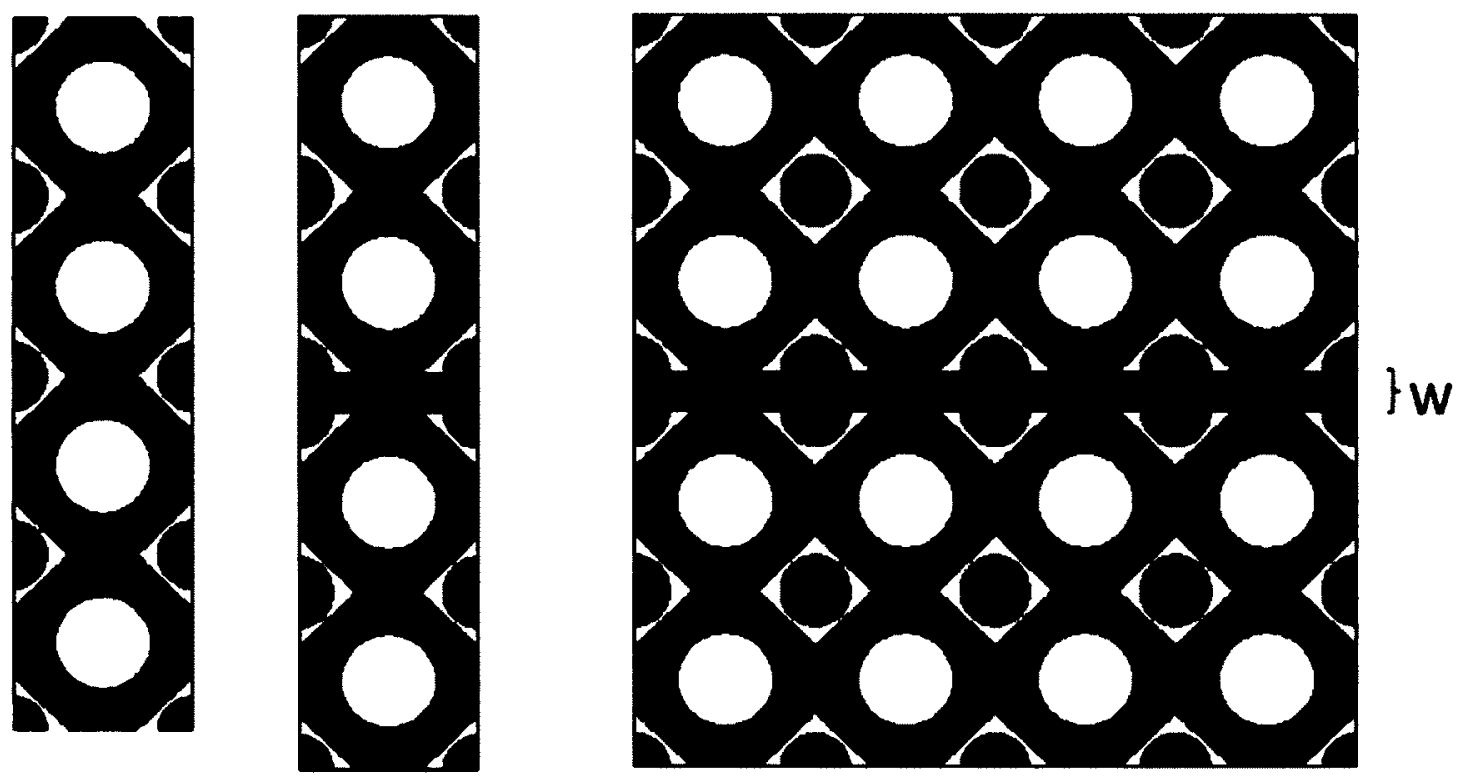

Figure 5.1: (left) $1 \times 4$ unit supercell, (middle) $1 \times 4$ supercell with a defect, and (right) the $4 \times 4$ array with the defect. Black represents the lower dielectric polymer, while white represents the higher dielectric material (silicon), and grey represents the waveguide material.

In figure 5.2 and 5.3, the band gaps for supercell without a defect (figure 5.1 left) for the TE and TM modes, respectively are shown. The band gaps are in the same location and are of the same width as for the single unit cell, see figure 4.6. The band gap for acoustic wave propagation in the supercell is shown in figure 5.4. As 441 plane waves were used to calculate the band structure of each basic unit cell, over 1600 waves were required to obtain accurate results for the band calculation for the supercell made up of 4 unit cells. Also, as the cell's number increase, more lines in the band diagrams will appear for the supercell compared to the unit cell. In addition, the symmetry points are compressed from point $\Gamma$ to $X$ and stretched for the symmetry points $X$ to $M$, and $M$ to $\Gamma$. That is because of the dimensions for the supercell is changed compared to the unit cell. 


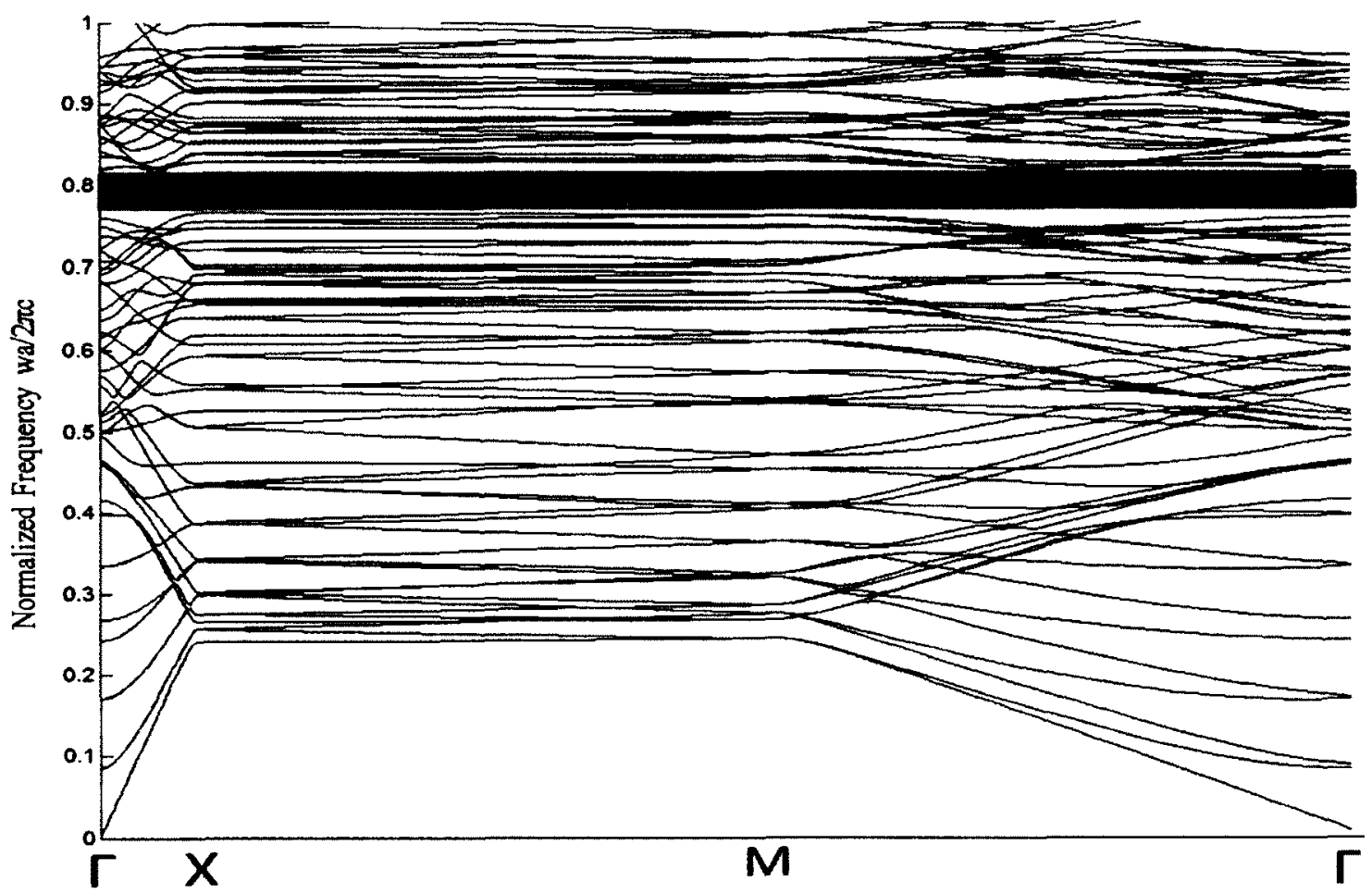

Figure 5.2: Optical TE band structure for a supercell (figure 5.1 left). The band gap is in the same location and of the same width as for the single unit cell (see figure 4.6 Top). 


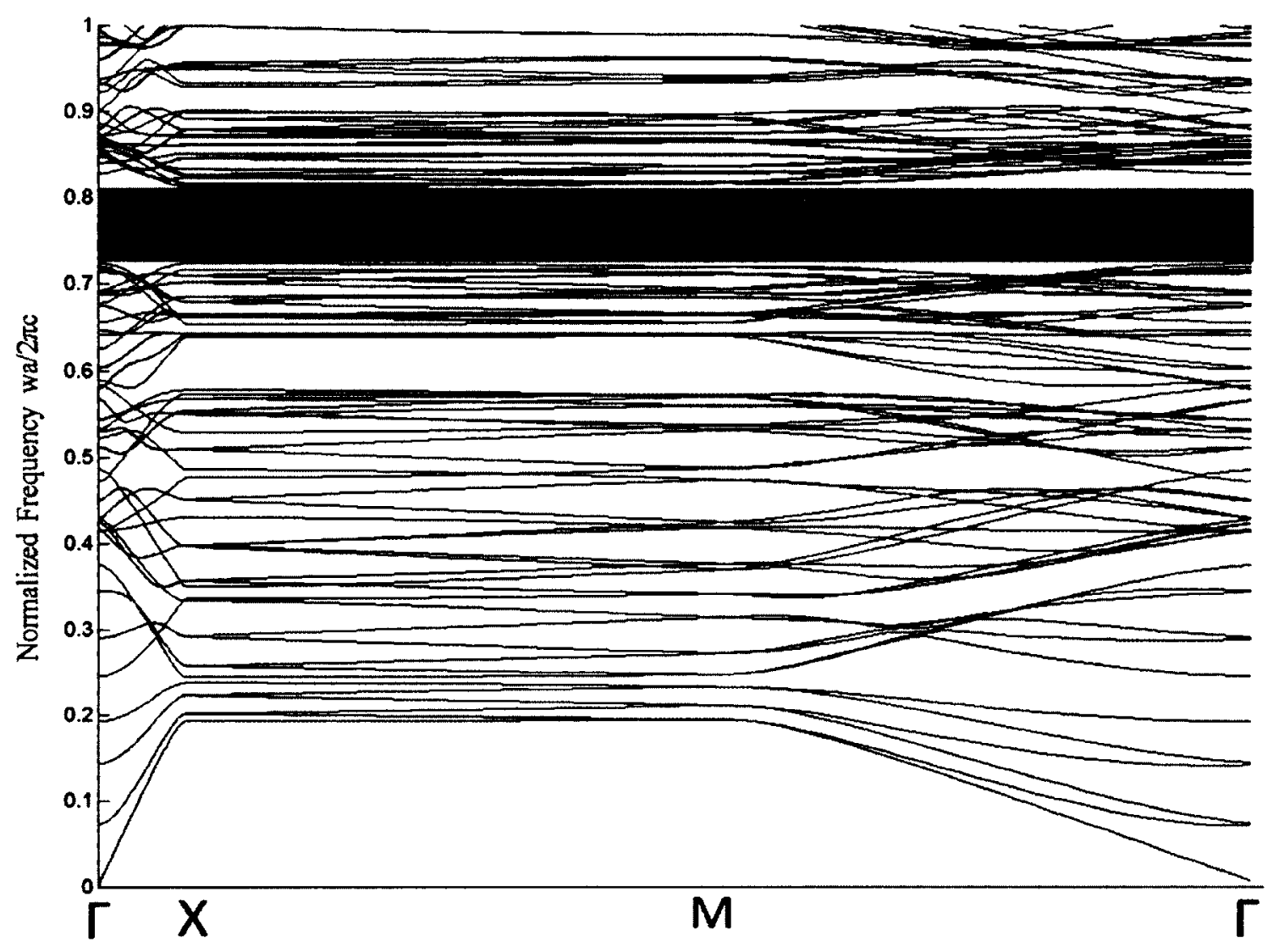

Figure 5.3: Optical TM band structure for a supercell (figure 5.1 left). The band gap is in the same location and of the same width as for the single unit cell (see figure 4.6 Bottom). 


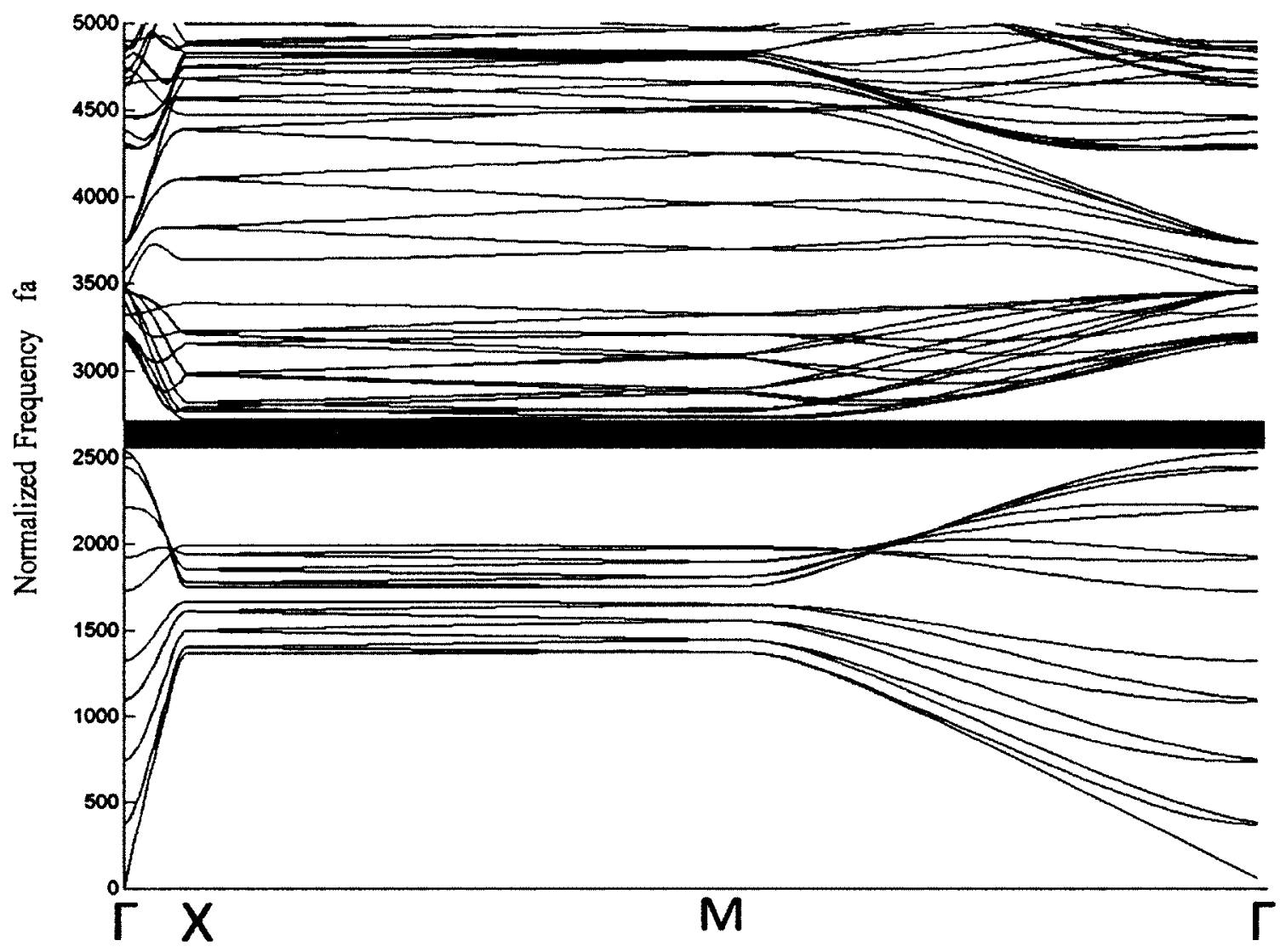

Figure 5.4: Acoustic band structure for a supercell without a defect state (figure 5.1 left). The band gap is in the same location and of the same width as for the single unit cell (see figure 4.7). 


\subsection{Line Waveguide}

Figure 5.1 (middle) shows a line defect (grey) in the supercell by introducing a gap along the center line of the structure. The line defect of width (W) can be filled with polymer, silicon or another material. The waveguide properties determined using the plane wave technique.The mode profile can be exmined by computing the eigenvector for the associated eigenfrequency

\subsection{Polymer as the Defect Material}

The width (W) of the line defect is a critical parameters of the waveguide. If $(W)$ is too small, no guided modes will be present. If $(\mathrm{W})$ is too large, the waveguide will be multimode. The material selected to fill the waveguide also plays a role and in this section is examined the polymer waveguide. A number of supercell computations were performed and the band structure determined verses waveguide width (W).the normalized frequncy 0.78 was selected and for $a=$ $1.2245 \mu \mathrm{m}$ gives $\lambda=1.55 \mu \mathrm{m}$ for optical waves and $\mathrm{f}=2.3 \mathrm{GHz}$ for acoustic. The plot of the

number of modes for TE, TM, and transverse acoustic is shown in figure 5.5. For single mode operation a width $\mathrm{W}=0.5$ was selected. 

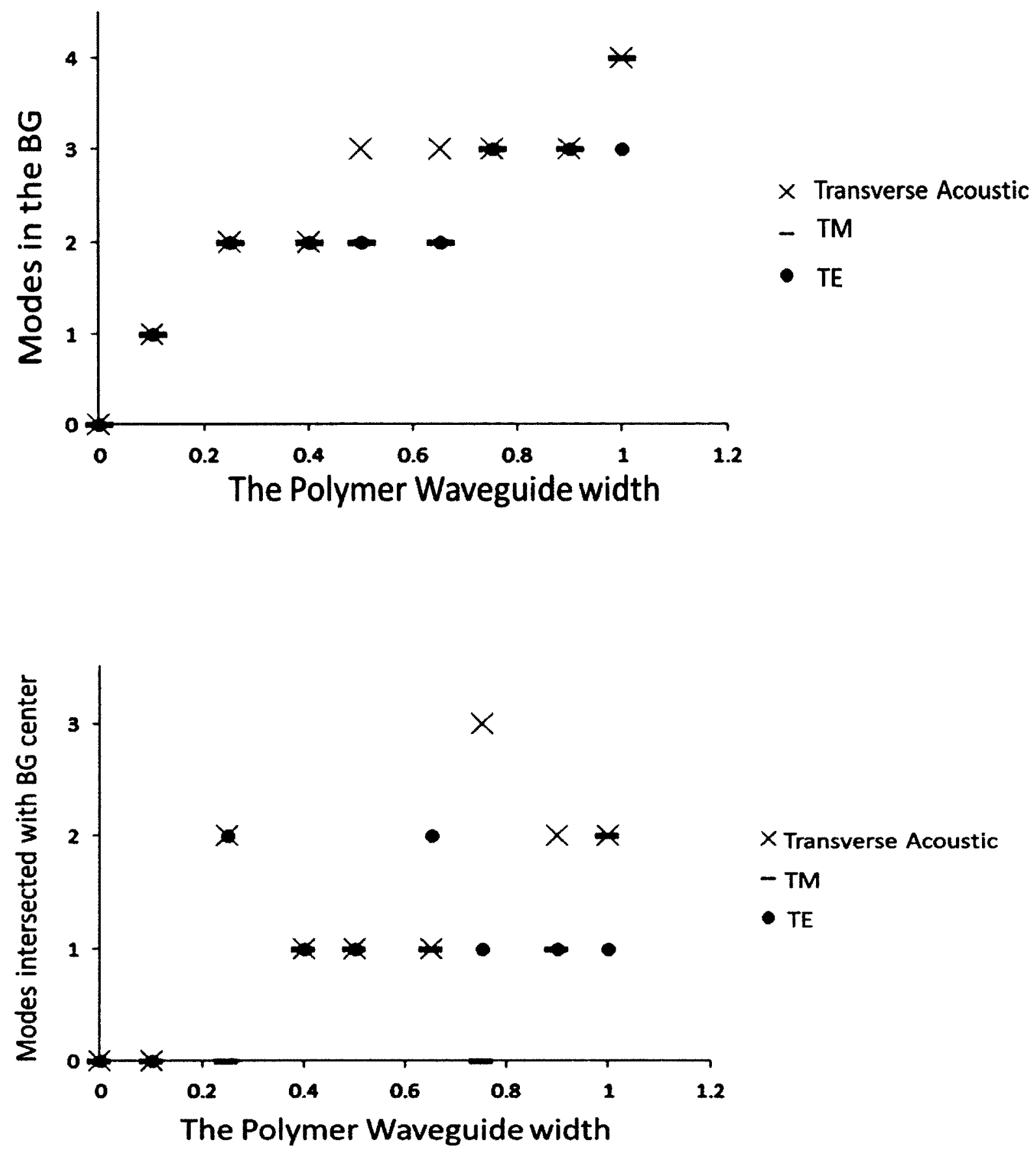

1

Figure 5.5: (Top) the number of modes inside the band gap as the polymer defect width increase. (Bottom) the number of modes intersected with the BG center as the polymer defect width increase. 
The band structure for TE and TM polarization in the supercell that has polymer in the $\mathrm{W}=0.5 \mathrm{a}$ width line defect region, are shown in figure 5.6 and figure 5.7 , respectively. The $\vec{k}$ vectors from the symmetry point $\Gamma$ to $\mathrm{X}$ are shown since the line defect along one of the lattice vectors. These figures show overlap band gaps and a single mode is presented within the band gaps. Also, the slopes are positive, which means the mode propagation is in the forward direction.

The eigenvectors can be found by substituting the specific eigenvalue into the eigenvalue equations (3.39), (3.43) and (3.55) for TE, TM, transverse respectively. For the TM guided mode, the normalized frequency of 0.78 corresponds to $\vec{k}$ vectors of $k_{0.78} \hat{x}$ and the expansion coefficients for this $\vec{k}$ vector associated with the eigenvalue are inserted. The eigenvector $K_{n, q}^{E_{z}}$ are used in:

$$
E_{z}(x, y)=\sum_{q=-\infty}^{\infty} \sum_{n=-\infty}^{\infty} K_{n, q}^{E_{z}} e^{-j \frac{2 \pi n}{a} x} e^{-j \frac{2 \pi q}{a} y} e^{-j k_{x} x}
$$

and produce the spatial field profile shown in figure 5.8. Grey scale image shows a mode strongly confined in the line defect region and small field value at the top and bottom of the supercell. on other side For TE, the eigenvector calculations process is the same, however, the calculations are performed for $H_{z}$ instead of $E_{z}$. The grey scale image figure 5.9 shows a confined mode in the line defect region. Examination of figures (5.8) and (5.9) shows that, the TM mode is more confined than the TE mode. This can be traced back to the band structure diagrams of figure (5.6) and (5.7). The TM band gap is large and the value of $0.78 \mathrm{a}$ corresponds to the gap center. 


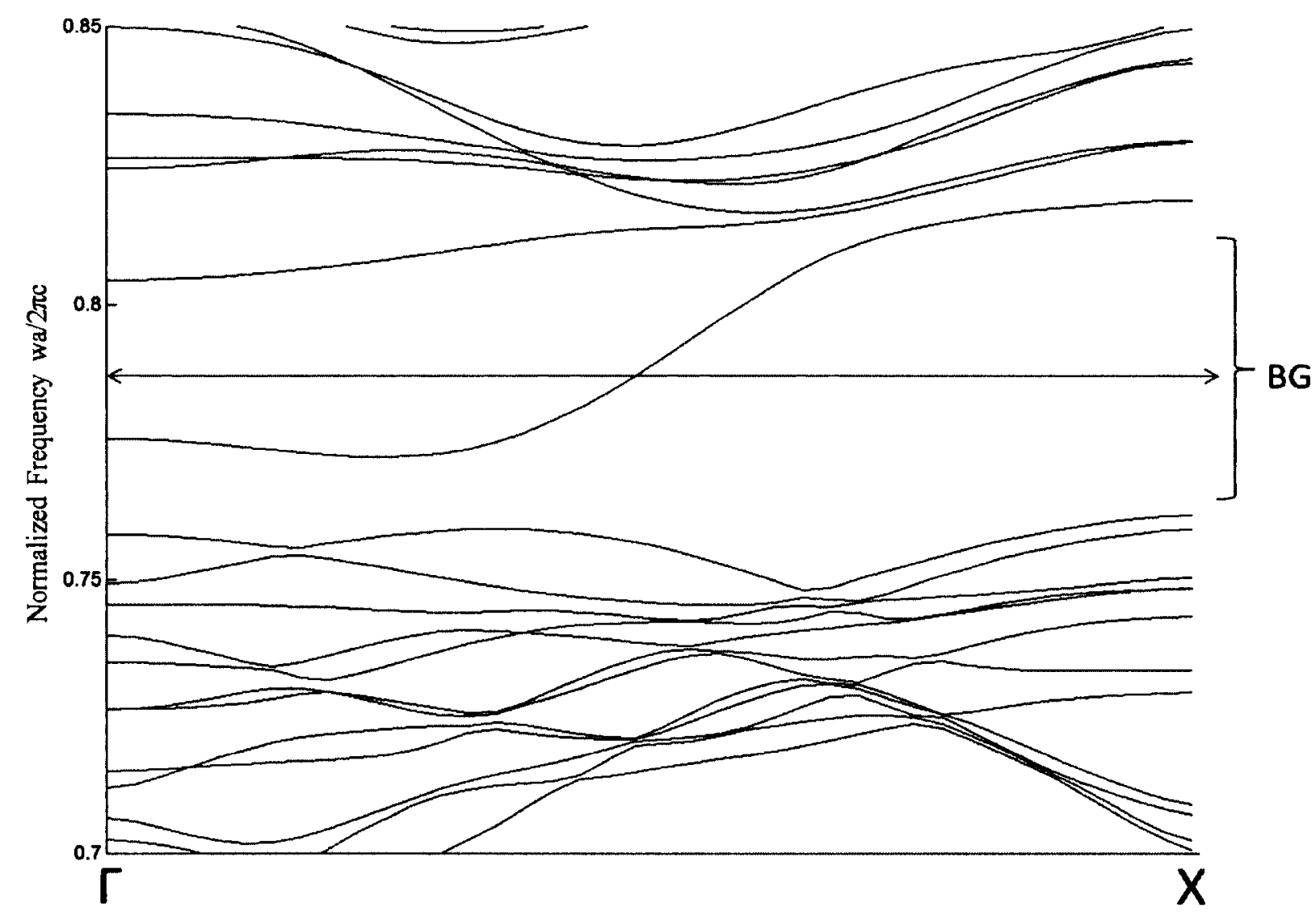

Figure 5.6: TE mode, $\Gamma$ to $\mathrm{X}$ band structure for the structure that has polymer in the defect region, the width of the defect is $\mathrm{W}=0.5 \mathrm{a} .3721$ plane waves were used for this calculation. 


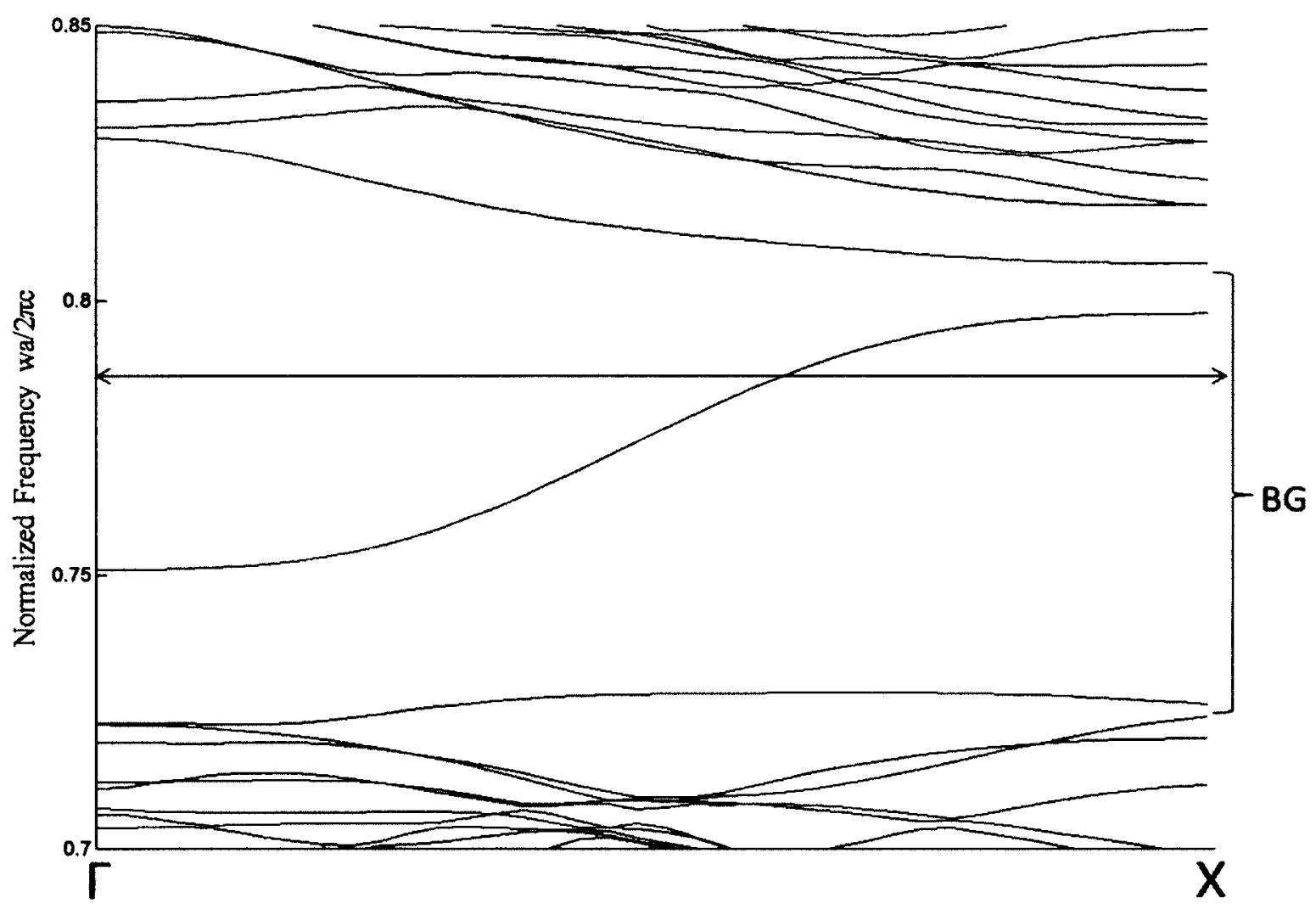

Figure 5.7: TM mode, $\Gamma$ to $\mathrm{X}$ band structure for the structure that has polymer in the defect region, the width of the defect is $\mathrm{W}=0.5 \mathrm{a} .3721$ plane waves were used for this calculation. 

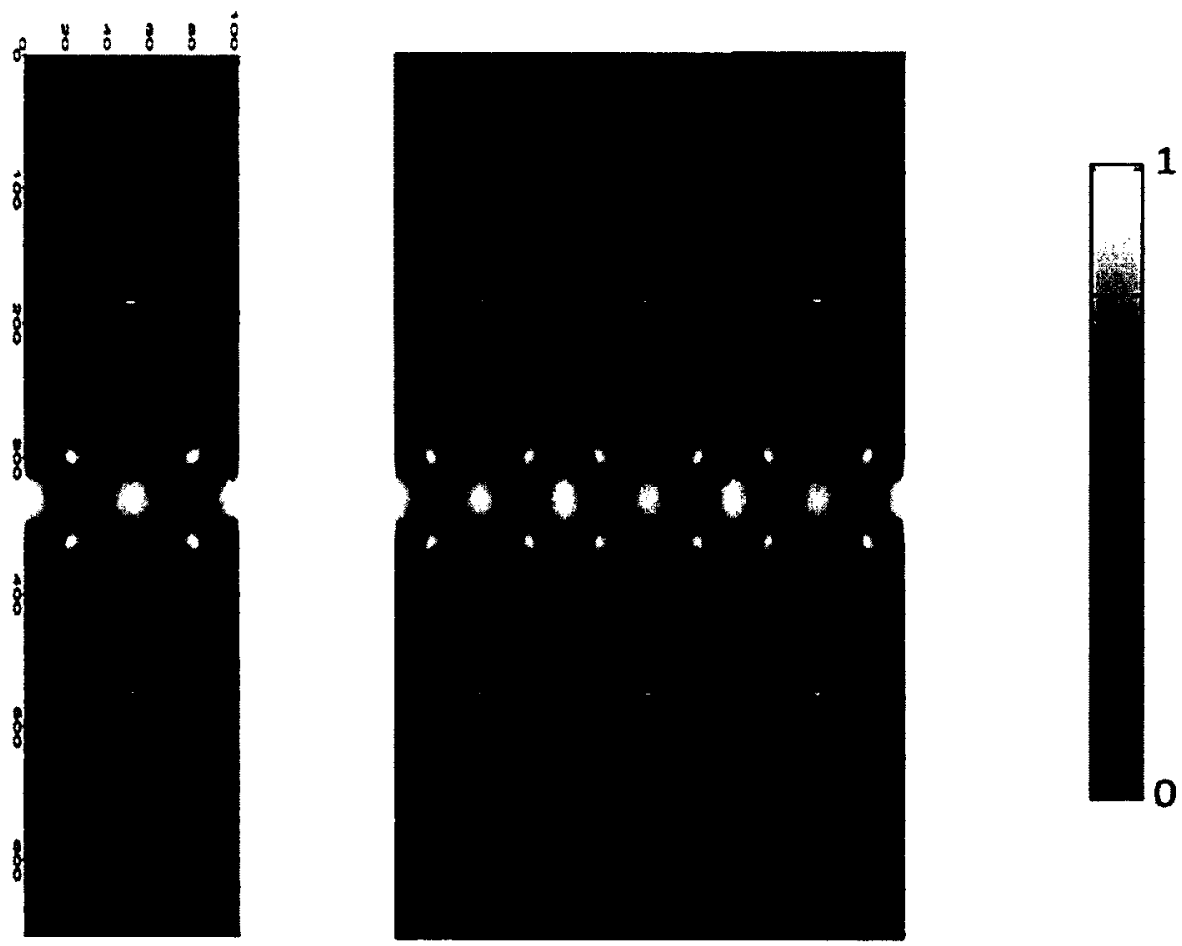

Figure 5.8: $\mathrm{TM}$ mode profile at $\mathrm{NOBG}=0.78$ for the structure that has polymer in the defect region, the width of the defect is $\mathrm{W}=0.5 \mathrm{a} .3721$ plane waves were used. 

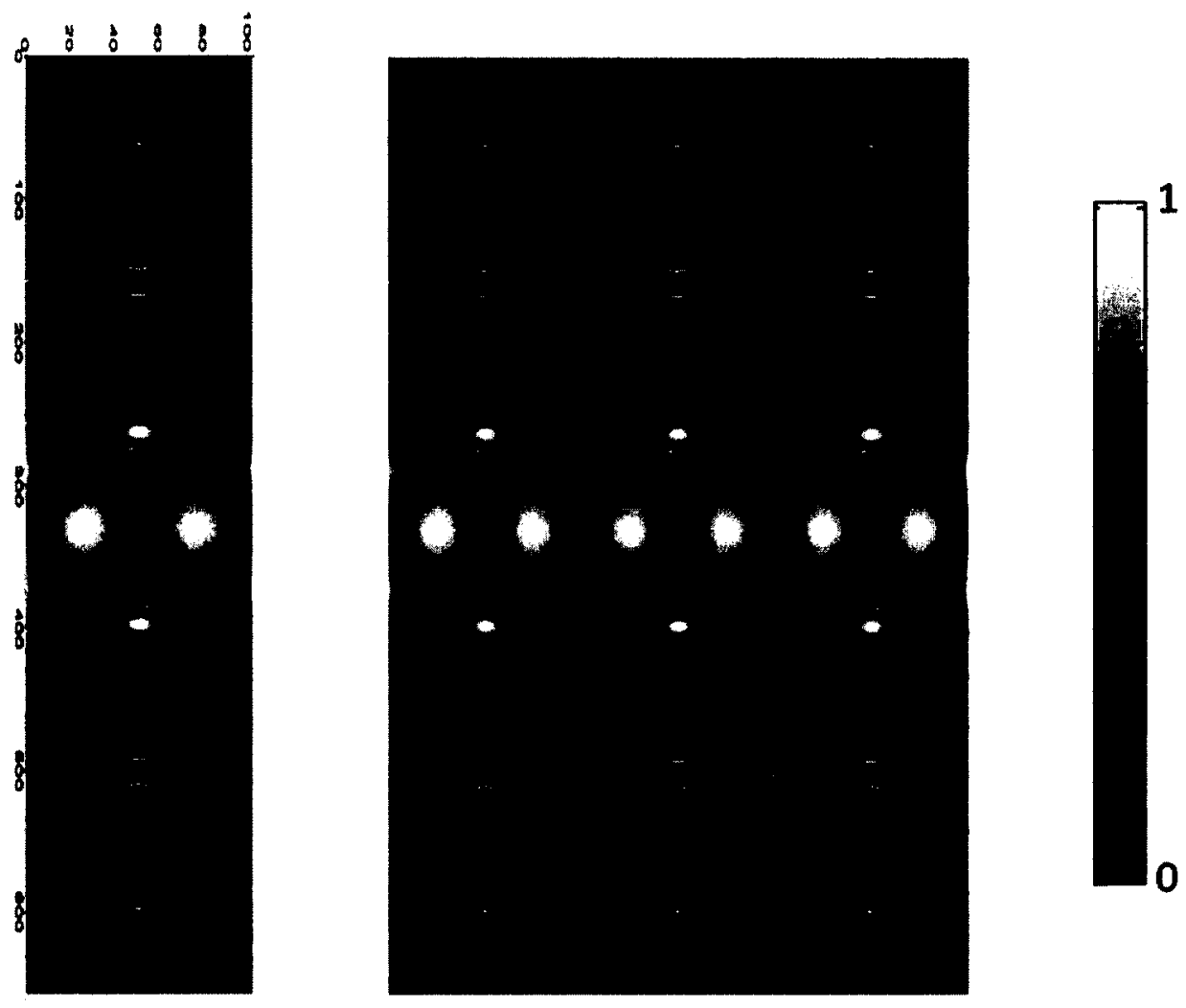

Figure 5.9: $T E$ mode profile at $\mathrm{NOBG}=0.78$ for the structure that has polymer in the defect region, the width of the defect is $W=0.5 \mathrm{a} .3721$ plane waves were used.

In the case of the transvers acoustical wave the supercells was increased to a size of $8 \times 1$. Figure 5.10 shows the $\Gamma$ to $\mathrm{X}$ band structure and single band line in the band gap for the transverses acoustic wave using 5041 plane waves. The mode profile shown in figure 5.11 is at normalized frequency $\mathrm{fa}=2680$ and is calculated by inserting the eigenvalue into equation 3 . 
With $\vec{k}=k_{2680} \hat{x}$. More plane waves were used to obtain accurate results (around 5041 plane wave).

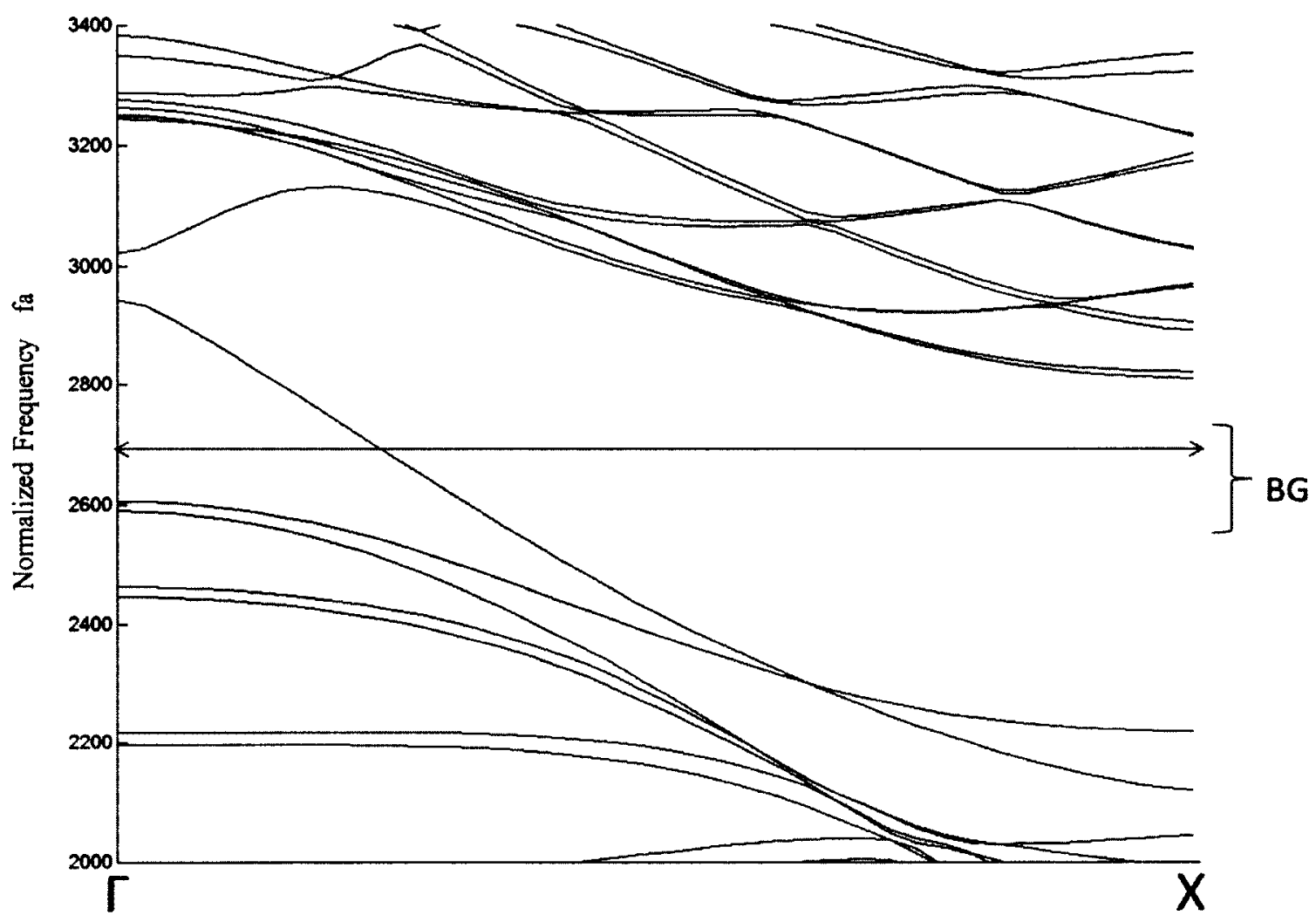

Figure 5.10: Acoustic transvers mode band structure for the structure that has polymer in the defect region, the width of the defect is $w=0.5 \mathrm{a} .5041$ plane waves were used. 

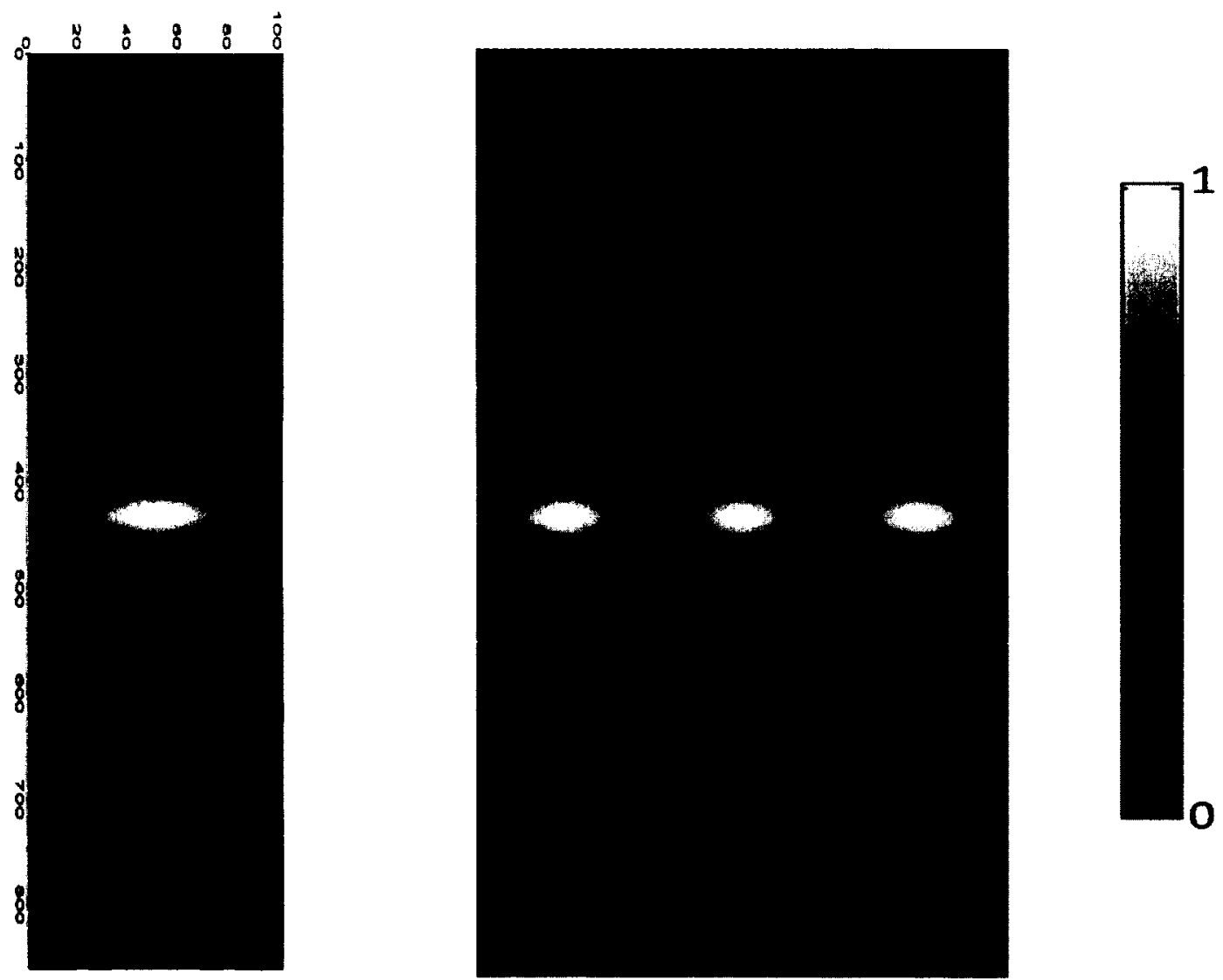

Figure 5.11: Acoustic transfer mode profile at $\mathrm{NABG}=2680$ for the structure that has epoxy in the defect region, the width of the defect is $w=0.5 \mathrm{a} .5041$ plane waves were used.

\subsection{Silicon as the Line Waveguide}

Silicon can be used as the defect material in the gap width and is convenient when the phoxonic crystal is connected to a silicon-waveguide, However, it was not easy to find the defect state for the acoustic wave encountering a small defect width. As shown in figure (5.12) a width of one unit cell was thus used to find the single mode state for acoustic waves. This means that many modes will exist for optical waves (TE and TM ). 

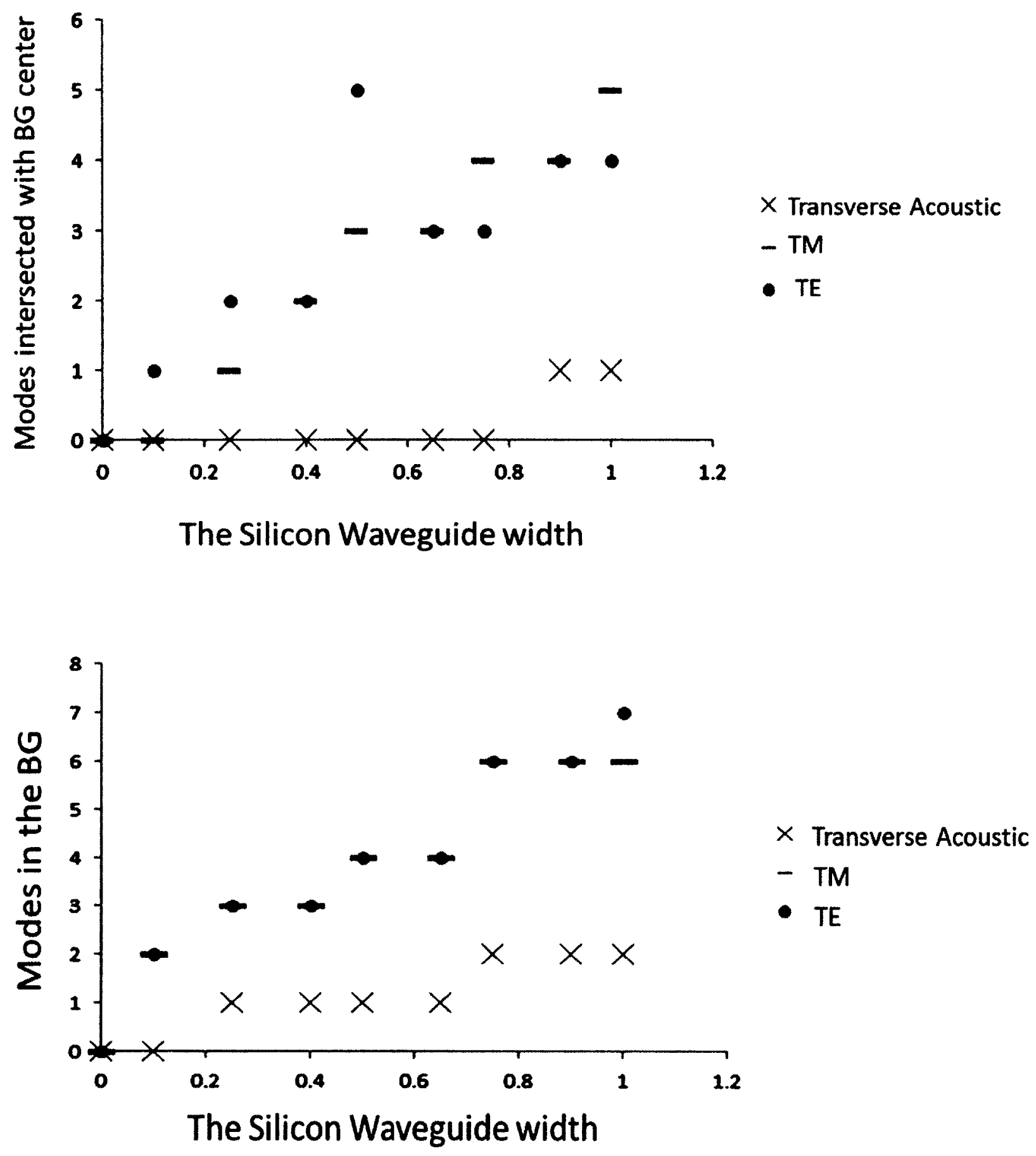

87| Chapter 5: Simultaneous Waveguide for Optical and Acoustic Waves 
Figure 5.12: (Top) the number of modes inside the band gap as the silicon defect width increase. (Bottom) the number of modes intersected with the BG center as the silicon defect width increase.

About 5041 plane waves were used to generate the acoustical data in the figures 5.5 and 5.12 , and at normalized frequency value $(f a=2667.2)$ the intersection of the modes is considerd. While for optical date 3741 plane waves are used, and the normalized value $w a / 2 \pi c=0.78$ is the value to which the intersection with mode is considered. 


\section{Conclusions}

The main purpose of this thesis was to study the effectiveness of different crystal structures in opening large simultaneous optical and acoustic band gaps, and to study the defect state and waveguide properties of such structures. The main structure studied was a siliconpolymer (solid-solid, low-contrast dielectric) composite. As complete optical band gaps are normally very difficult to open in this material, many different structures had to be evaluated before the desired properties were obtained.

The Plane Wave was used to obtain more accurate results. The results obtained by this method were found largely to be in agreement with published results. The similarity of the equations used for optical and the transfer acoustic wave propagation suggests that the optical propagation simulation code can easily be adapted - with a few modifications - to the study of acoustic propagation.

By using a composite unit cell composed of both high and low dielectric materials, the difficulties of opening complete simultaneous optical and acoustic band gaps are overcome. The composite structure studied has the appearance of two phoxonic structures - one exhibiting a TM mode band gap, the other a TE mode band gap - combined together. The full structure exhibits a complete optical band gap and transverse acoustic band gap.

The defect state and waveguide properties were investigated by increasing the local separation between adjacent unit cells along a lattice vector and filling the gaps with epoxy, or silicon. A good localized TM mode was found for a high-normalized frequency of $0.78 \mathrm{a}$. Frequency is scalable, so by changing the lattice constant the localized frequency can be specified. In addition, a low-normalized acoustic frequency was localized in the same 
waveguide. However, because this frequency is in $\mathrm{GHz}$ range, more cells normal to the lattice vector are needed to insure a tighter mode for low frequencies.

Studying the band structure of the finite thickness (plane) of the structure or for different geometry lattice structure such as triangle can be the future work that can develop the result of this work. Finite thickness phoxonic structure is more realizable for practical applications. Also, plane phoxonic structure will insure the confinement of optical and acoustic wave in third dimension. On other hand, the different lattice geometry could help to open larger band gaps. This is because of the reduction of the symmetry the structure will have with different geometry structure. In addition, studying complete acoustic polarizations band structure is required to give more valuable enhancement for this work. 


\section{Appendix A}

Simplifying and reduction of the summation terms of the Eigenvalue Equations

$$
\begin{gathered}
\frac{1}{\varepsilon_{r}}\left(\frac{\partial^{2} \vec{E}_{y}}{\partial z^{2}}\right)=\frac{1}{c^{2}} \frac{\partial^{2} \vec{E}_{y}}{\partial t^{2}} \\
\frac{1}{\varepsilon_{r}}=\sum_{m=-\infty}^{\infty} K_{m}^{\varepsilon_{r}} e^{-j \frac{2 \pi m}{a} z} \\
E_{y}=\sum_{n=-\infty}^{\infty} K_{n}^{E_{y}} e^{-j \frac{2 \pi n}{a} z} e^{-j k_{z} z} e^{-j w t}
\end{gathered}
$$

Inserting (A2) and (A3) in (A1)

$$
\begin{aligned}
& \sum_{m=-\infty}^{\infty} K_{m}^{\varepsilon_{r}} e^{-j \frac{2 \pi m}{a} z} \sum_{n=-\infty}^{\infty} K_{n}^{E_{y}}\left(\frac{2 \pi n}{a}+k_{z}\right)^{2} e^{-j \frac{2 \pi n}{a} z} e^{-j k_{z} z} e^{-j \omega t}=\frac{w^{2}}{c^{2}} \sum_{n=-\infty}^{\infty} K_{n}^{E_{y}} e^{-j \frac{2 \pi n}{a} z} e^{-j k_{z} z} e^{-j w t} \\
\Rightarrow & \sum_{m=-\infty}^{\infty} \sum_{n=-\infty}^{\infty} K_{m}^{\varepsilon_{r}} K_{n}^{E_{y}} e^{-j \frac{2 \pi m}{a} z}\left(\frac{2 \pi n}{a}+k_{z}\right)^{2} e^{-j \frac{2 \pi n}{a} z} e^{-j k_{z} z} e^{-j w t}=\frac{w^{2}}{c^{2}} \sum_{n=-\infty}^{\infty} K_{n}^{E_{y}} e^{-j \frac{2 \pi n}{a} z} e^{-j k_{z} z} e^{-j w t}
\end{aligned}
$$

The common terms on the right side and left side can be canceled, therefore the equation above can be written as 


$$
\sum_{m=-\infty}^{\infty} \sum_{n=-\infty}^{\infty} K_{m}^{\varepsilon_{r}} K_{n}^{E_{y}} e^{-j \frac{2 \pi m}{a} z}\left(\frac{2 \pi n}{a}+k_{z}\right)^{2} e^{-j \frac{2 \pi n}{a} z}=\frac{w^{2}}{c^{2}} \sum_{n=-\infty}^{\infty} K_{n}^{E_{y}} e^{-j \frac{2 \pi n}{a} z}
$$

Multiplying this equation by orthogonal function $e^{j \frac{2 \pi p}{a} z}$, where $\mathrm{p}$ is integer number, and then

$$
\begin{aligned}
& \text { integrating each side over a unit cell } \frac{1}{a} \int_{-\frac{a}{2}}^{\frac{a}{2}} \cdots d z \\
& \qquad \sum_{m=-\infty}^{\infty} \sum_{n=-\infty}^{\infty} K_{m}^{\varepsilon_{r}} K_{n}^{E_{y}}\left(\frac{2 \pi n}{a}+k_{z}\right)^{2} e^{-j \frac{2 \pi}{a}(n+m-p) z}=\frac{w^{2}}{c^{2}} \sum_{n=-\infty}^{\infty} K_{n}^{E_{y}} e^{-j \frac{2 \pi}{a}(n-p) z} \\
& \frac{1}{a} \sum_{m=-\infty}^{\infty} \sum_{n=-\infty}^{\infty} K_{m}^{\varepsilon_{r}} K_{n}^{E_{y}}\left(\frac{2 \pi n}{a}+k_{z}\right)^{2} \int_{\frac{-a}{2}}^{\frac{a}{2}} e^{-j \frac{2 \pi}{a}(n+m-p) z} d z=\frac{1}{a} \frac{w^{2}}{c^{2}} \sum_{n=-\infty}^{\infty} K_{n}^{E_{y}} \int_{\frac{-a}{2}}^{\frac{a}{2}} e^{-j \frac{2 \pi}{a}(n-p) z} d z
\end{aligned}
$$

Using the orthognality of the expansion function the real solution will be only for $n+m=p$ and $\mathrm{n}=\mathrm{p}$. For nontrivial solution, $\mathrm{p}$ can take only one value. Therefore,the summations can be reduced from three summation to one summation.

$$
\sum_{n=-\infty}^{\infty} K_{m-n}^{\varepsilon_{r}} K_{n}^{E_{x}}\left(\frac{2 \pi n}{a}+\vec{k}_{z}\right)^{2}=\frac{w^{2}}{c^{2}} K_{m}^{E_{x}}
$$


93 Appendix A: Simplifying and reduction of the summation terms of the Eigenvalue Equations 


\section{Appendix B}

MATLAB Code for Opical calculation TE and TM.

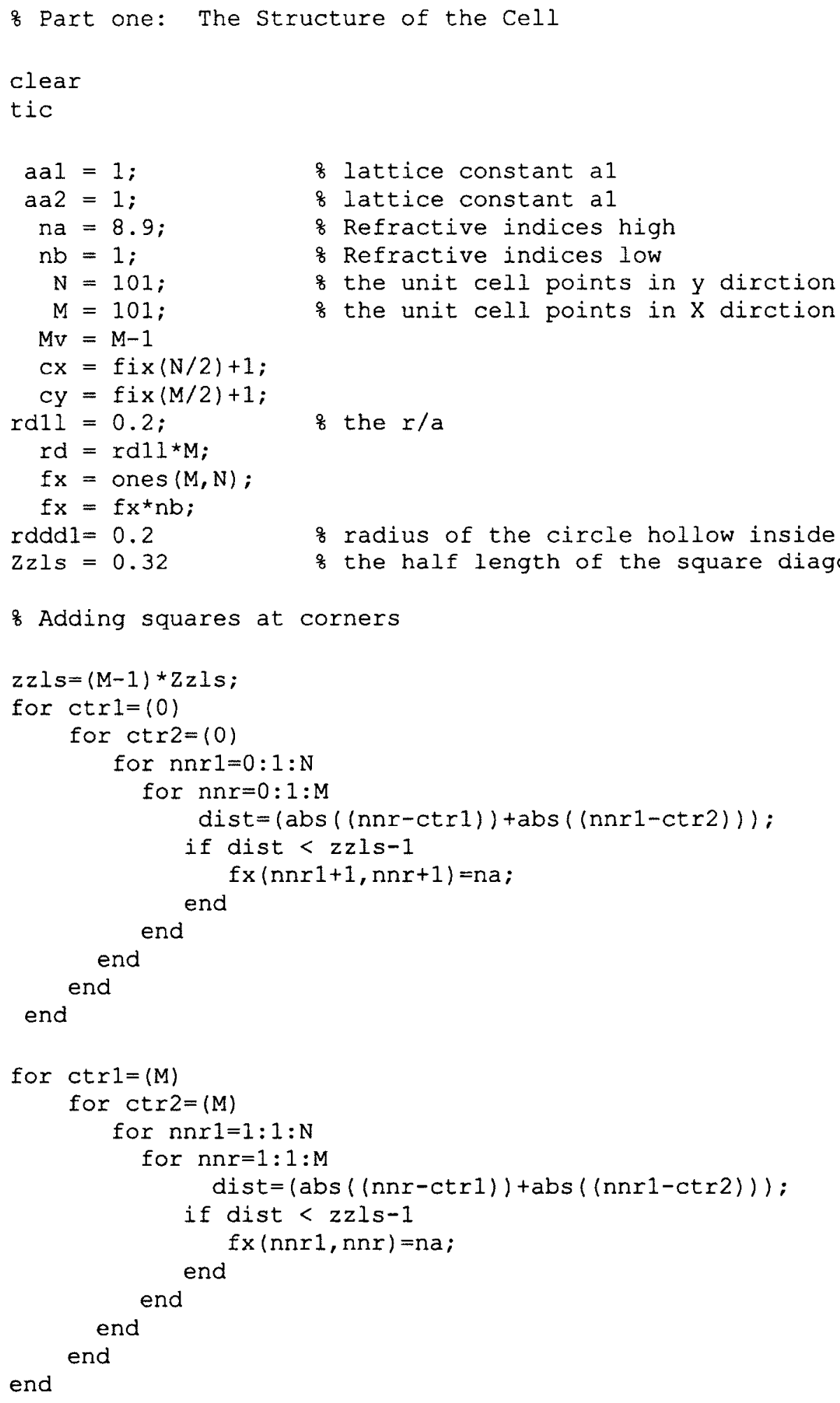

94 |Appendix B: MATLAB Code for Opical calculation TE and TM. 


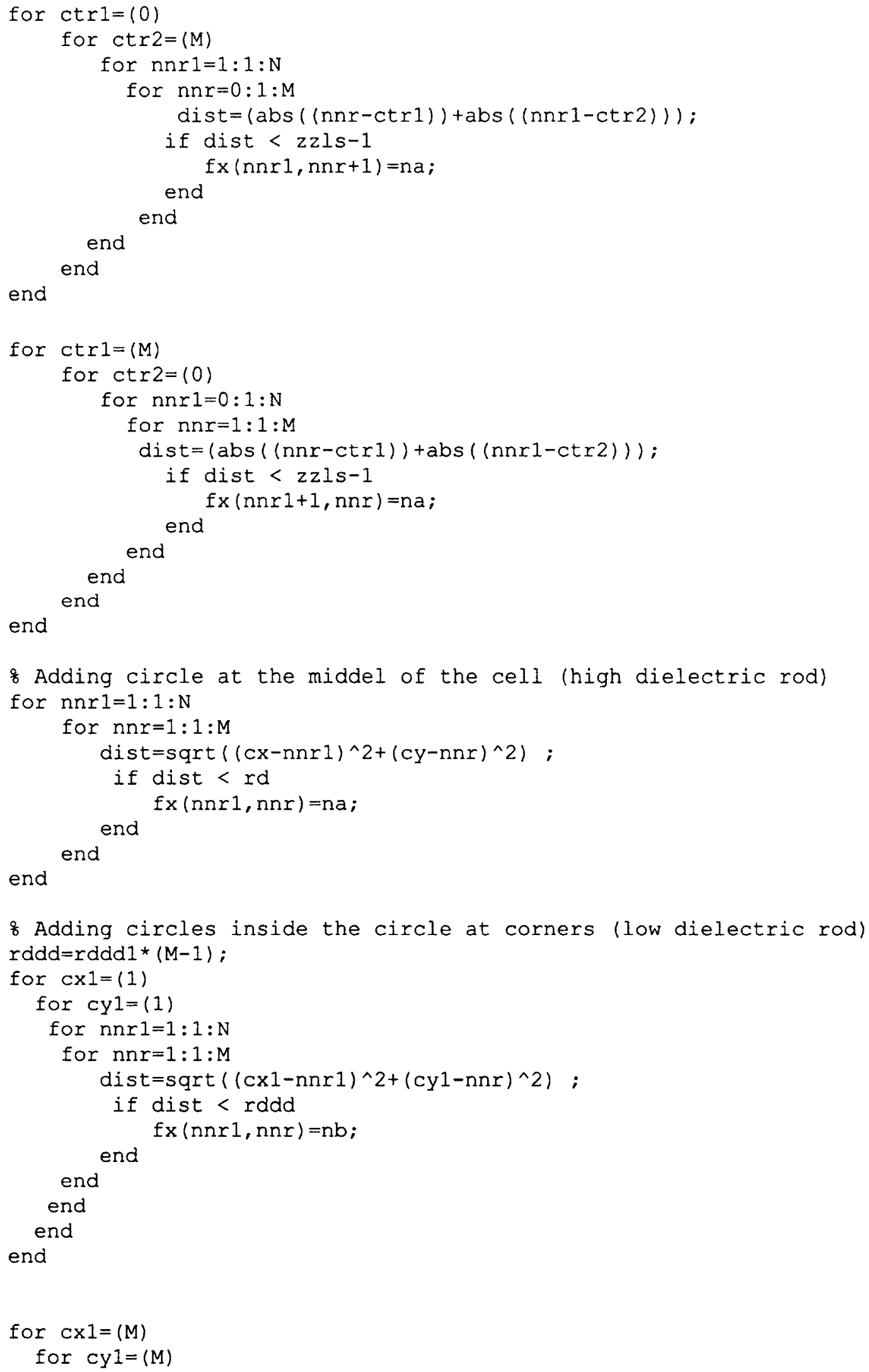

95 |Appendix B: MATLAB Code for Opical calculation TE and TM. 


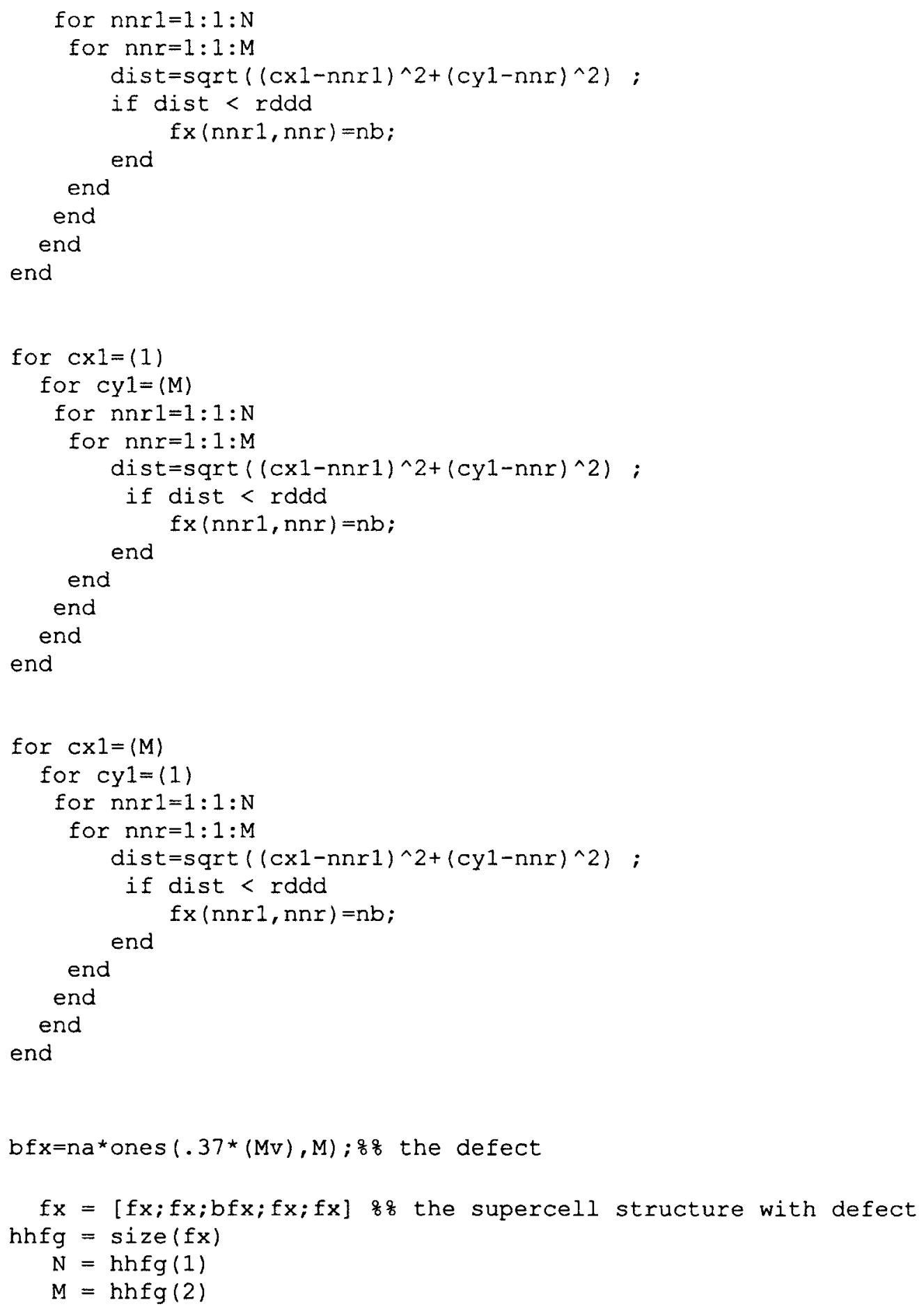




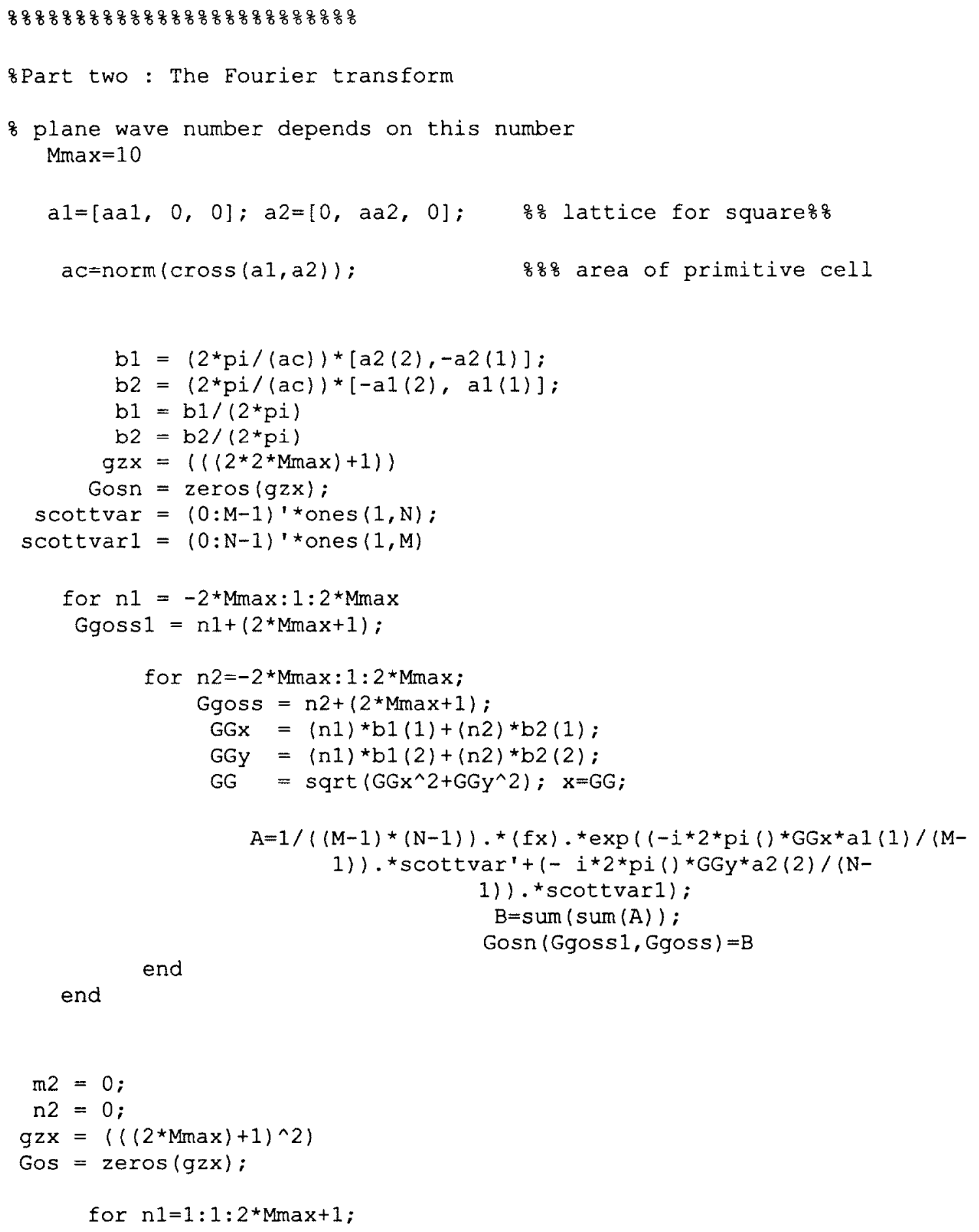

97 |Appendix B: MATLAB Code for Opical calculation TE and TM. 


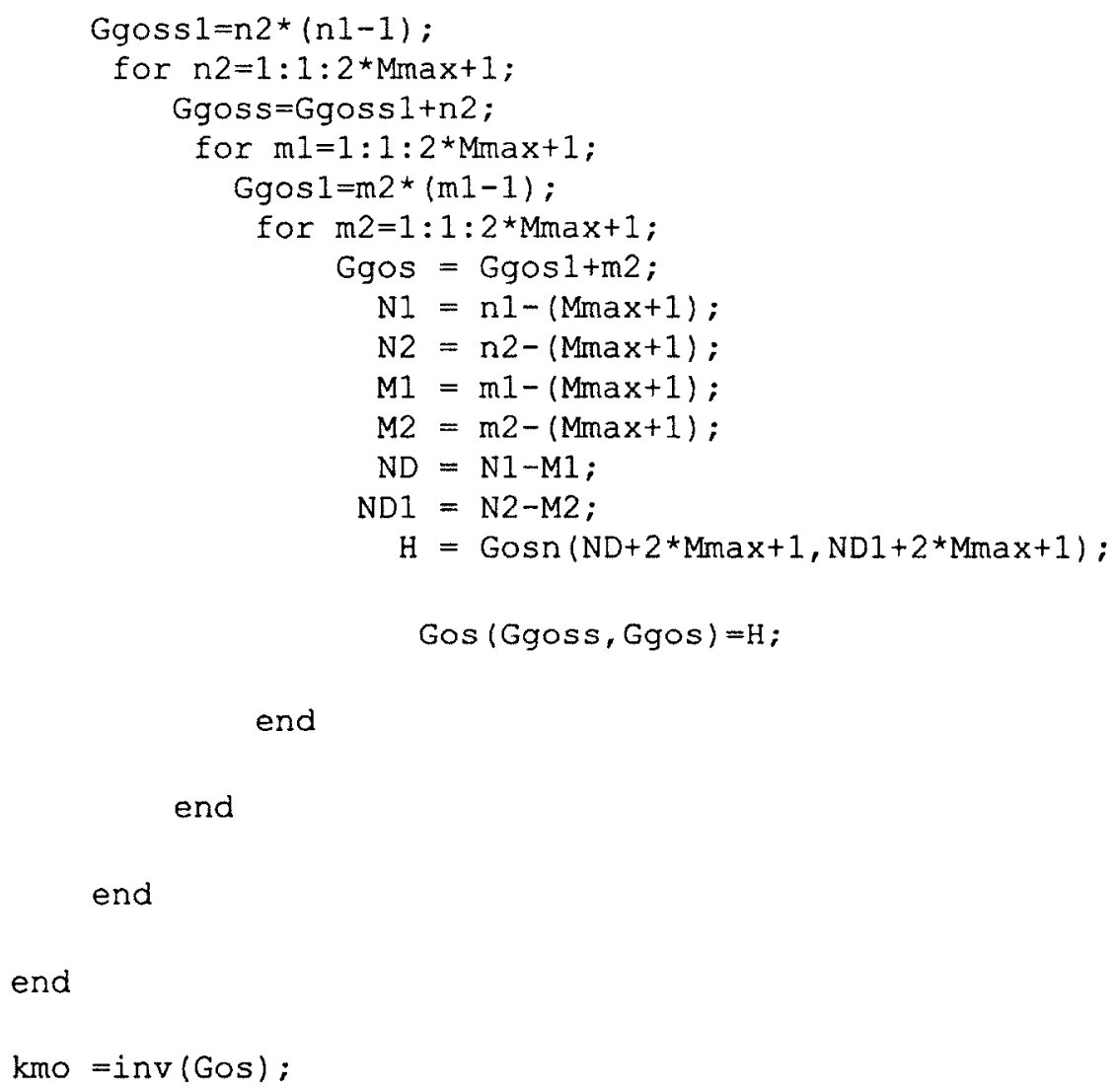




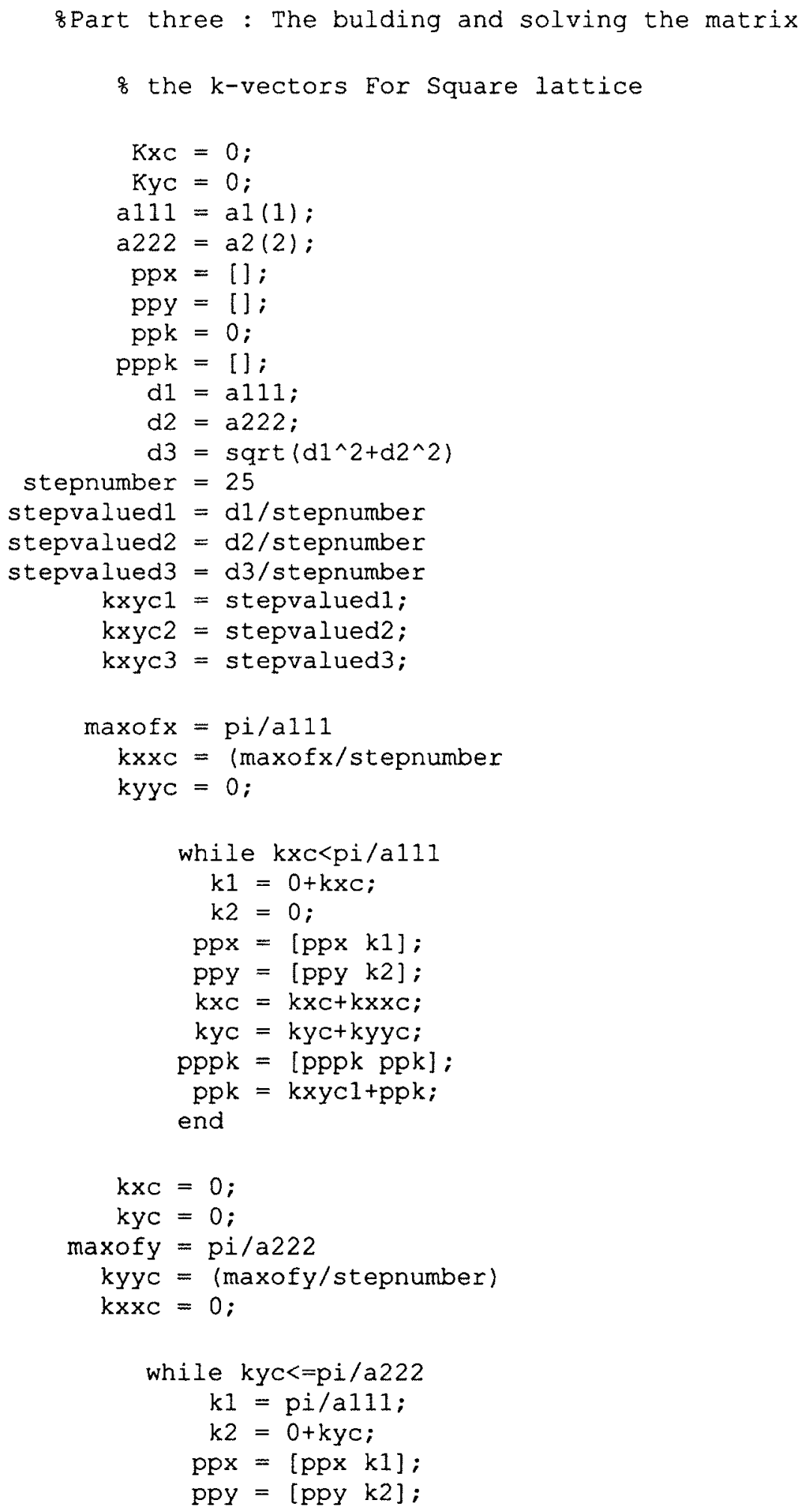

99 |Appendix B: MATLAB Code for Opical calculation TE and TM. 


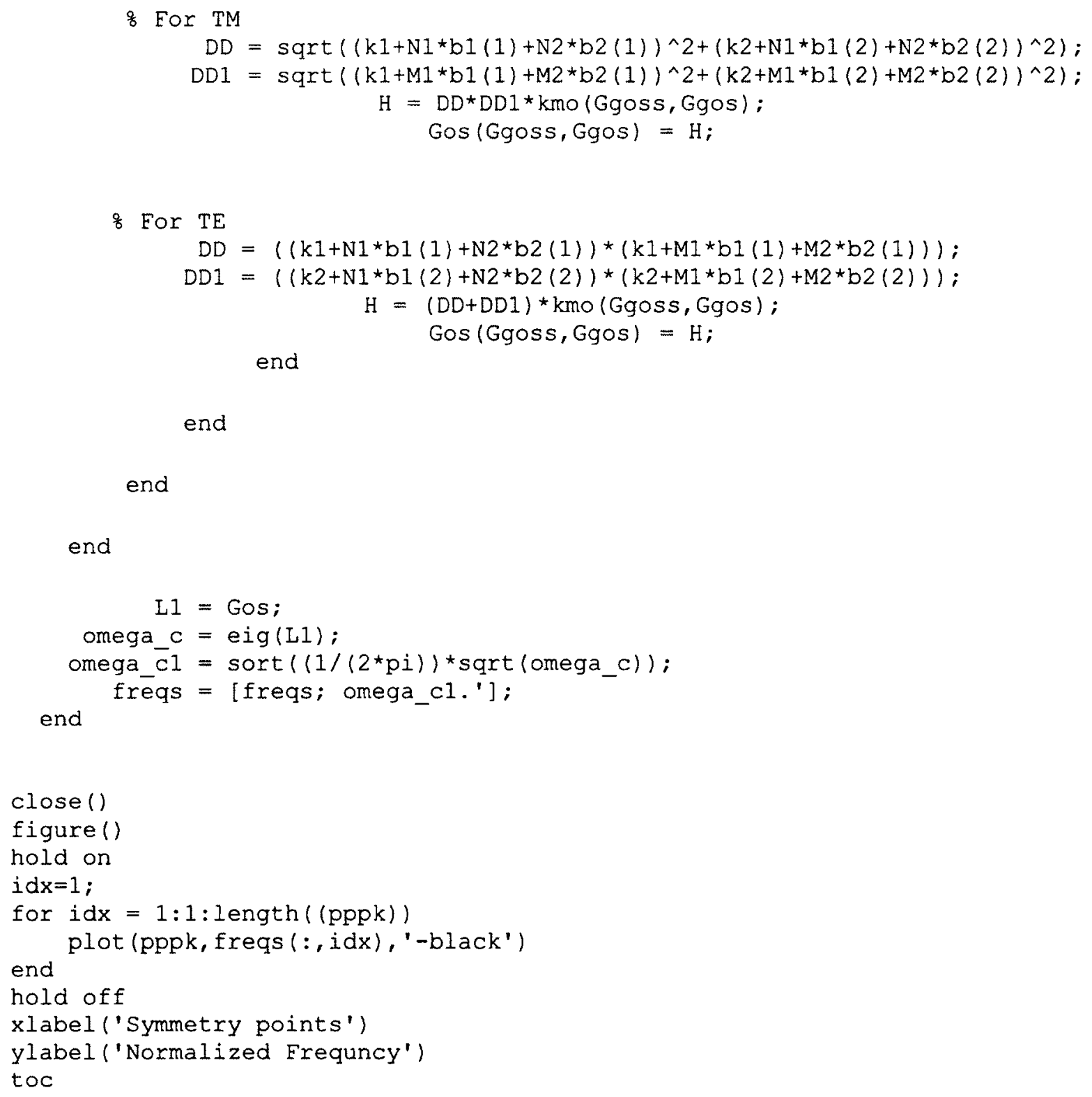




\section{Appendix C}

MATLAB Code for Acoustic calculation shear mode.

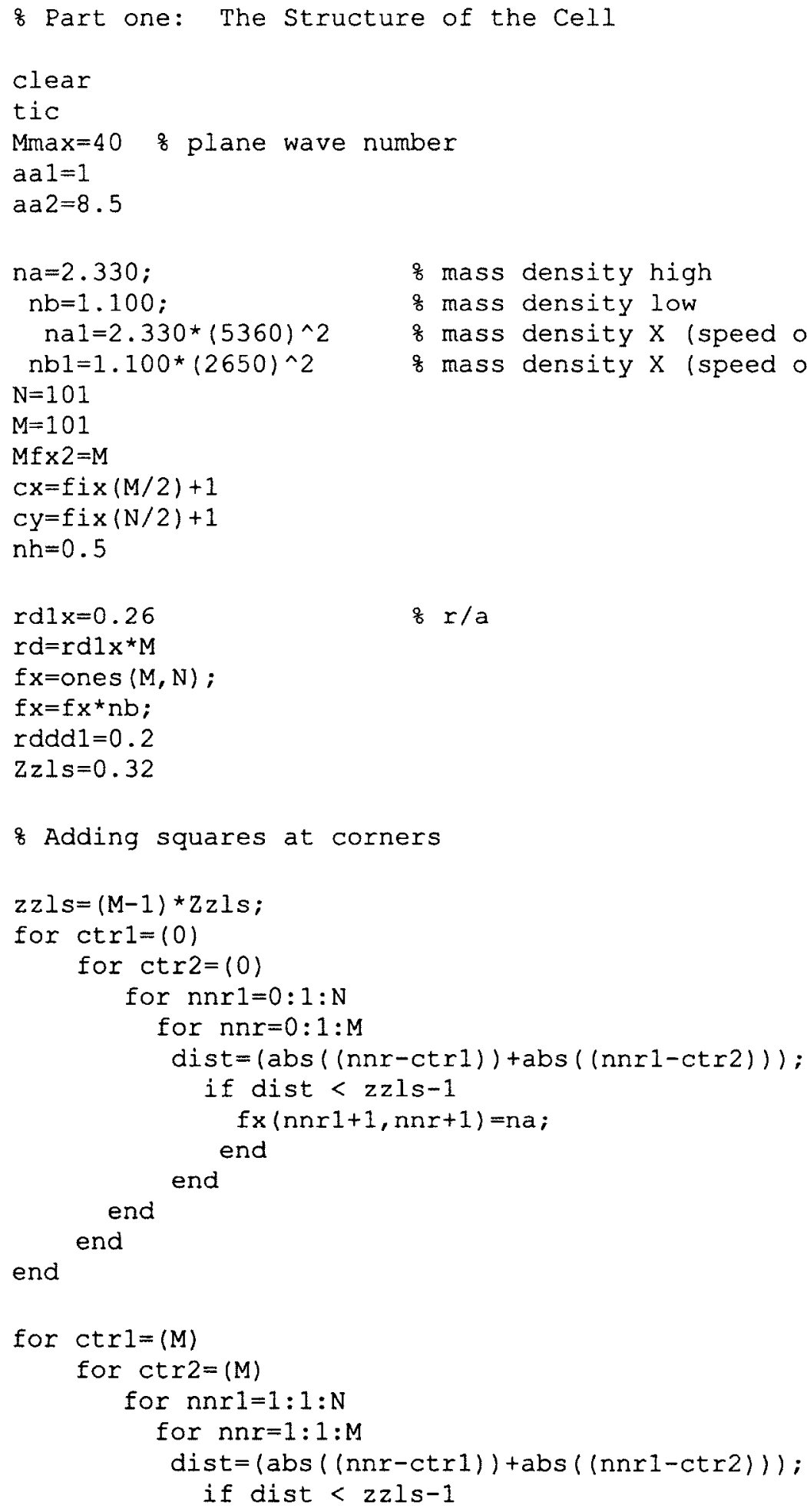

102 Appendix C: MATLAB Code for Acoustic calculation shear mode. 


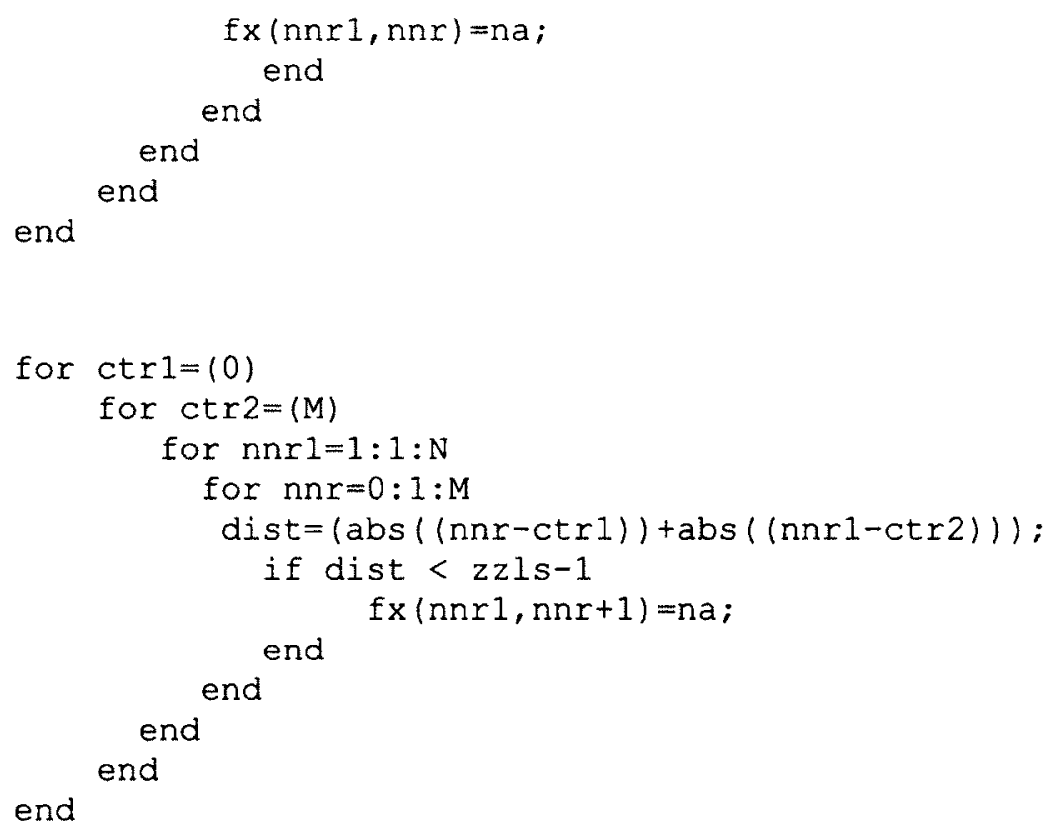

103 |Appendix C: MATLAB Code for Acoustic calculation shear mode. 


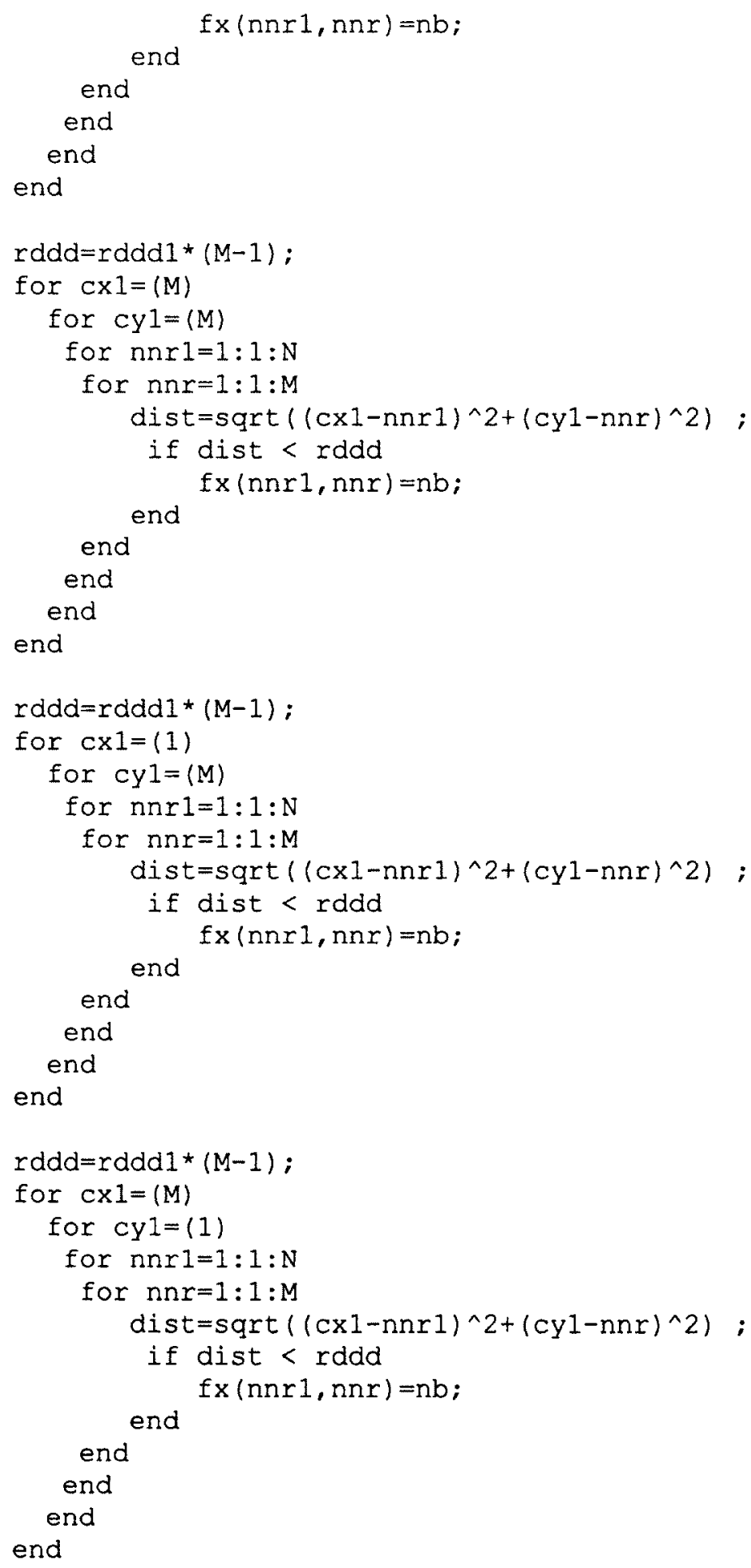

104 IAppendix C: MATLAB Code for Acoustic calculation shear mode. 


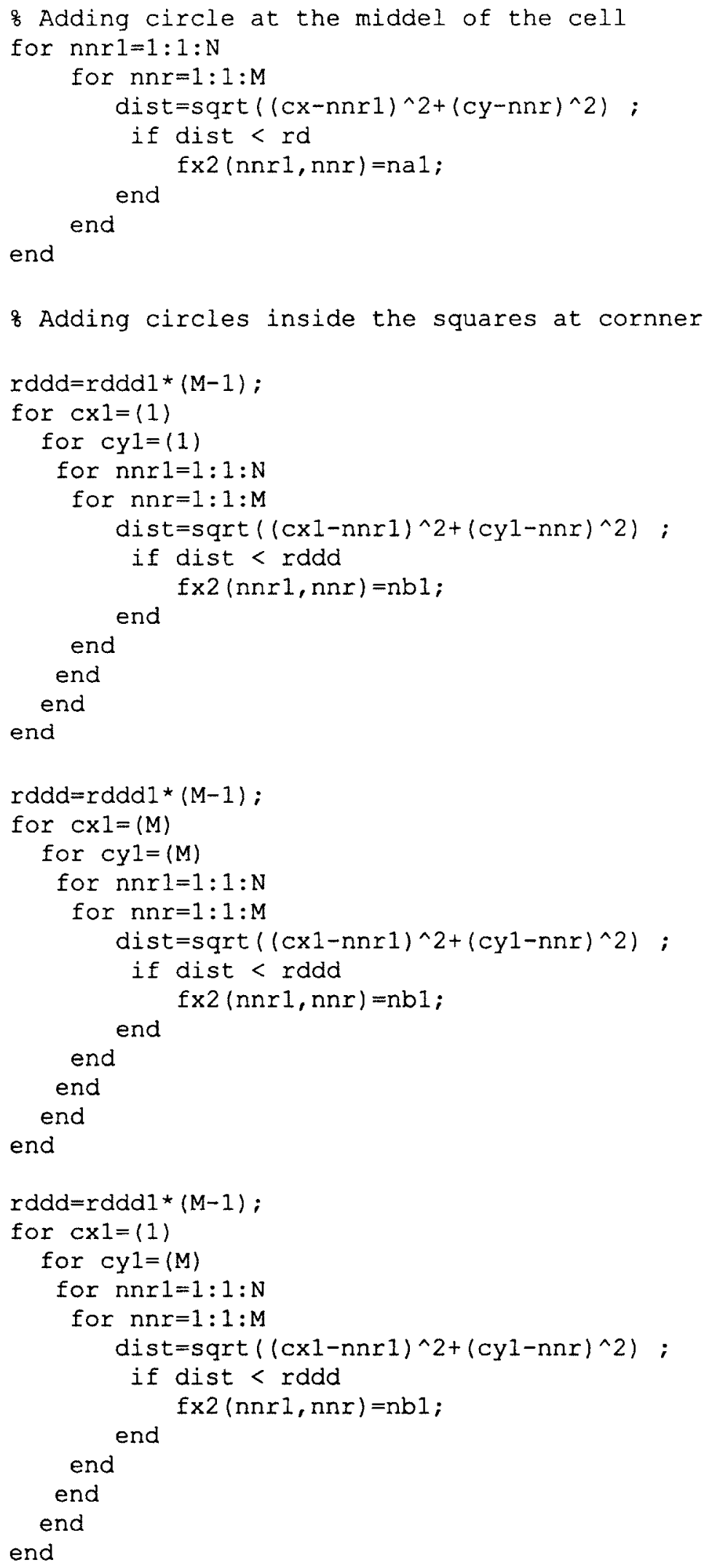

106 Appendix C: MATLAB Code for Acoustic calculation shear mode. 


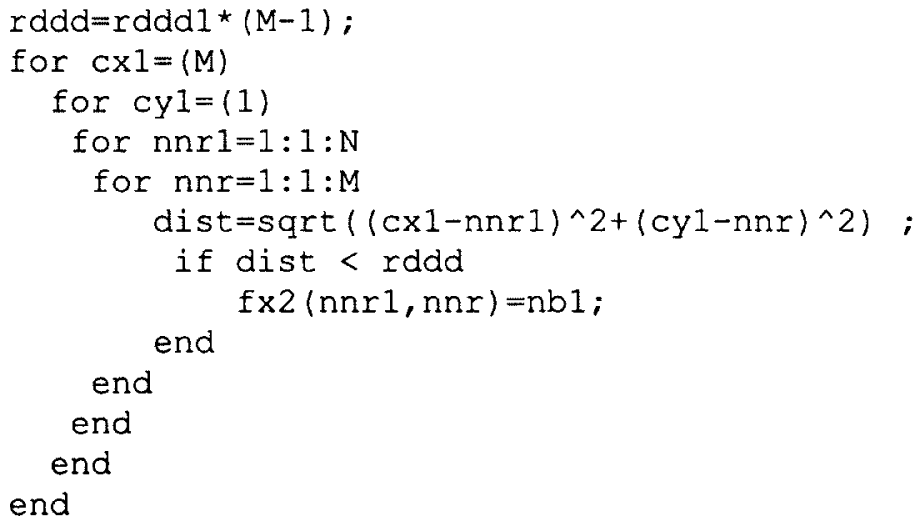




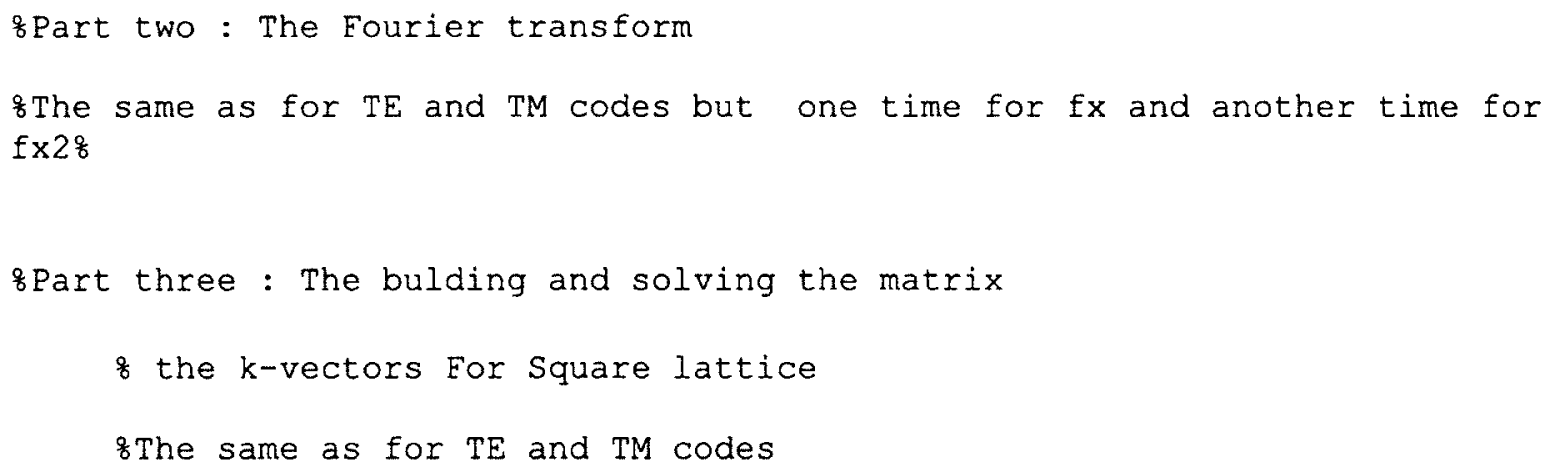

\& This part is written based on what polarizations is needed $\mathrm{DD}=((\mathrm{k} 1+\mathrm{N} 1 * \mathrm{~b} 1(1)+\mathrm{N} 2 * \mathrm{~b} 2(1)) *(\mathrm{k} 1+\mathrm{M} 1 * \mathrm{~b} 1(1)+\mathrm{M} 2 * \mathrm{~b} 2(1)))$;

end

end

end

108 |Appendix C: MATLAB Code for Acoustic calculation shear mode. 


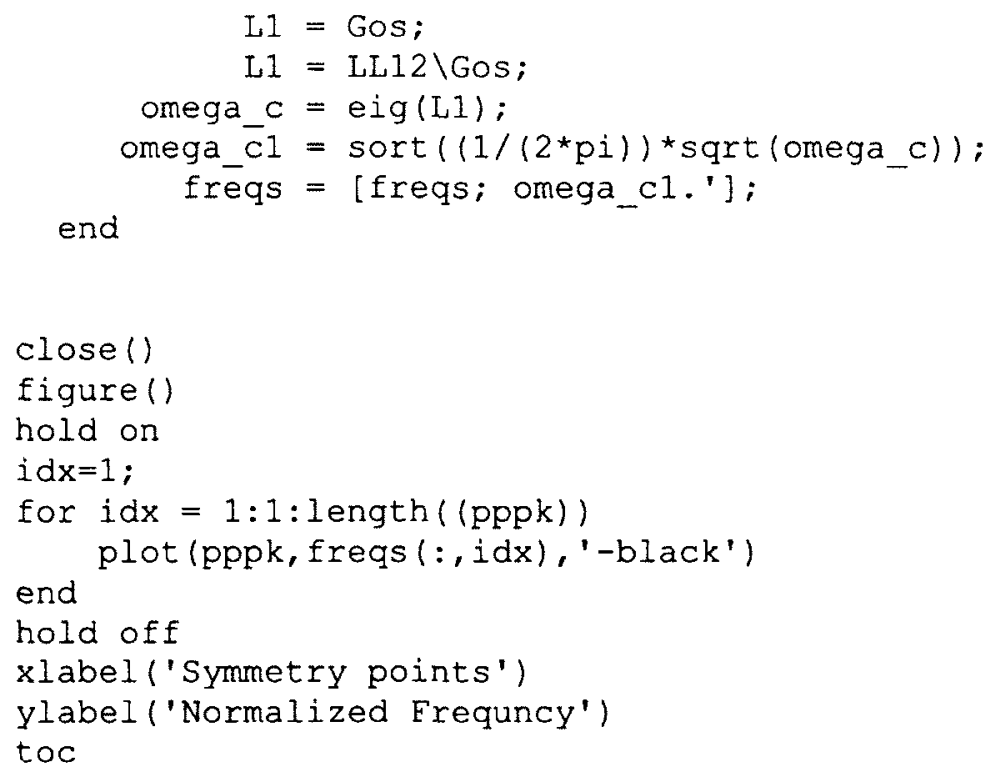

109 |Appendix C: MATLAB Code for Acoustic calculation shear mode. 


\section{References}

[1] Yablonovitch, E., "Inhibited Spontaneous Emission in Solid-State Physics and Electronics," Phys. Rev. Lett., Vol. 58, 2059-2062 (1987).

[2] Joannopoulos D., J., Meade D., R., Winn N., J., Photonic Crystals: Molding the Flow of Light, Princeton University Press (1995).

[3] Ben S., Sanjay B., Solid State Electronic Devices, Pearson Prentice-Hall(2009).

[4] V. Narayanamurti, H. L. Störmer, M. A. Chin, A. C. Gossard, and W. Wiegmann," Selective Transmission of High-Frequency Phonons by a Superlattice: The "Dielectric" Phonon Filter" Phys. Rev. Lett. 43, 2012-2016 (1979).

[5] Jean-Michel L., Henri B., Dominique P., Vincent B., Jean-Michel G.,and Daniel M., Photonic Crystals: Towards Nanoscale Photonic Devices, Springer-Verlag Berlin Heidelberg (2008).

[6] M. Loncar, D. Nedeljkovi, T. Doll, J. Vuckovic, A. Scherer, and T. P. Pearsall, "Waveguiding in planar photonic crystals," Appl. Phys. Lett. 77(13), 1937-1939 (2000).

[7] Y. Akahane, T. Asano, B. S. Song, and S. Noda, "High-Q photonic nanocavity in a twodimensional photonic crystal," Nature 425(6961), 944-947 (2003).

[8] M. Koshiba, "Wavelength division multiplexing and demultiplexing with photonic crystal waveguide couplers," J. Lightwave Technol. 19(12), 1970-1975 (2001).

[9] Zhi-Yuan Li, Lan-Lan Lin, Ben-Yuan Gu!, Guo-Zhen Yang "Photonic band gaps in anisotropic photonic crystals," Physica B 279, 159-161(2000).

[10] Nobuhiko Susaa, "Large absolute and polarization-independent photonic band gaps for various lattice structures and rod shapes" J. Appl. Phys., 91( 6), 3501-3510( 2002)

[11] M. Maldovan, and E. L. Thomas, "Simultaneous complete elastic and electromagnetic band gaps in periodic structures," Appl. Phys. B 83(4), 595-600 (2006).

[12] M. Maldovan, and E. L. Thomas, "Simultaneous localization of photons and phonons in two-dimensional periodic structures," Appl. Phys. Lett. 88(25), 251907 (2006).

[13] S. Sadat-Saleh, S. Benchabane, F. I. Baida, M. P. Bernal, and V. Laude, "Tailoring simultaneous photonic and phononic band gaps," J. Appl. Phys. 106(7), 074912 (2009).

[14] T. Carmon, H. Rokhsari, L. Yang, T. J. Kippenberg, and K. J. Vahala, "Temporal behavior of radiation-pressureinduced vibrations of an optical microcavity phonon mode," Phys. Rev. Lett. 94(22), 223902 (2005). 
[15] M. Eichenfield, R. Camacho, J. Chan, K. J. Vahala, and O. Painter, "A picogram- and nanometre-scale photoniccrystal optomechanical cavity," Nature 459(7246), 550-555 (2009).

[16] A. V. Akimov, Y. Tanaka, A. B. Pevtsov, S. F. Kaplan, V. G. Golubev, S. Tamura, D. R. Yakovlev, and M. Bayer, "Hypersonic modulation of light in three-dimensional photonic and phononic band-gap materials," Phys. Rev. Lett. 101(3), 033902-033905 (2008).

[17] D. Caballero, J. Sanchez-Dehesa, C. Rubio, R. Martinez-Sala, J. V. Sanchez-Perez, F. Meseguer,and J. Llinares, "Large two-dimensional sonic band gaps" Rev. E. 60(6), 6316-6319 (1999).

[18] K. M. Ho, C. T. Chan, and C. M. Soukoulis, "Existence of a photonic gap in periodic dielectric structures," Phys.Rev.Lett. 65, 3152-3155 (1990).

[19] Y. S. Chan, C. T. Chan, and Z.Y. Liu., "Photonic Band Gaps in Two Dimensional Photonic Quasicrystals"Phys. Rev. Lett. 80,5(1998)

[20] S. John," Strong Localization of Photons in Certain Disordered Dielectric Superlattices “ Phys. Rev. Lett. 58, 2486 (1987).

[21] L. Rayleigh. On the maintenance of vibrations by forces of double frequency, and on the propagation of waves through a medium endowed with a periodic structure. Phil. Mag, 24(147):145-159, (1887)

[22] Z. Zhang and S. Satpathy," Electromagnetic Wave Propagation in Periodic Structures: Bloch Wave Solution of Maxwell's Equations" Phys. Rev. Lett. 65, 2650 (1990).

[23] W. M. Robertson, G. Arjavalingam, R. D. Meade, K. D. Brommer, A. M. Rappe, and J. D. Joannopoulos, "Measurement of photonic band structure in a two-dimensional periodic dielectric array "Phys. Rev. Lett. 68, 2023 (1992).

[24] Woldering L A, Tjerkstra R W, Jansen H V, Setija I D and Vos W.," Periodic arrays of deep nanopores made in silicon with reactive ion etching and deep UV lithography" Nanotechnology 19145304 (2008).

[25] Cheng C C, Arbet-Engels V, Scherer A and Yablonovitch E., "Nanofabricated three dimensional photonic crystals operating at optical wavelengths" Phys. Scr. T68 17 (1996).

[26] R. H. Olsson III and I. El-Kady. "Microfabricated phononic crystal devices and applications," Measurement science and technology, page 012002, (2009). 
[27] Campbell M, Sharp D N, HarrisonMT, Denning R G and Turberfield A J "Fabrication of photonic crystals for the visible spectrum by holographic lithography," Nature 40453 (2000).

[28] Straoscio and Dutta.,Phonons in Nanostructures, Cambridge University Press (2001). [29] M. S. Kushwaha, P. Halevi, L. Dobrzynski, and B. Djafari-Rouhani. "Acoustic band structure of periodic elastic composites”. Phys. Rev. Lett., 71(13):2022-2025, (1993).

[30] F. R. Montero de Espinosa, E. Jim_enez, and M. Torres. "Ultrasonic band gap in a periodic two-dimensional composite". Phys. Rev. Lett., pages 1208-1211, (1998).

[31] Gorishnyy T, Ullal C K, Maldovan M, Fytas G and Thomas E L "Hypersonic phononic crystals" Phys. Rev.Lett. 94 115501, (2005).

[32] Cheng W, Wang J, Jonas U, Fytas G and Stephanou N "Observation and tuning of hypersonic bandgaps in colloidal crystals" Nat. Mater. 5 830-6, (2006).

[33] A.W. Warner, D. L. White, and W. A. Bonner, "Acousto-opticlight deflectors using optical activity in paratellurite," J. Appl. Phys.,vol. 43, pp. 4489-4495, (1972).

[34] I. C. Chang, "Acousto-optic devices and applications," IEEE Trans. Sonics Ultrasonics, vol. SU-23, (1976).

[35] D. Maydan, "Acousto-optical pulse modulators," IEEE $J$. Quan- turn Electron., vol. QE-6, pp. 15-24, (1970).

[36] J.O. Vasseur, B. Djafari-Rouhani, L. Dobrzynski, M.S. Kushwaha,P. Halevi," Complete acoustic band gaps in periodic fibre reinforced composite materials: the carbon/epoxy composite and some metallic systems " J. Phys.: Condens. Matter. 6, 8759 (1994).

[37] MM Sigalas, "Elastic wave band gaps and defect states in two-dimensional composites," J. Acoust. Soc. Am. 101, 1256 (1997).

[38] M.M. Sigalas," Defect states of acoustic waves in a two-dimensional lattice of solid cylinders “J. Appl. Phys. 84, 3026 (1998).

[39] M.M. Sigalas, E.N. Economou,"Attenuation of multiple-scattered sound" Europhys. Lett. 36, 241 (1996).

[40] M.S. Kushwaha, P. Halevi, G. Mart'nez, L. Dobrzynski, B. Djafari-Rouhani," Theory of acoustic band structure of periodic elastic composites " Phys. Rev. B 49, 2313 (1994). 
[41] Hua Ma, Shaobo Qu, and Zhup Xu, "Photonic crystals based on acoustic-optic effects" J. appl. Phys. 103, 104904 (2008). 